\title{
A PRELIMINARY STUDY OF CLIMATIC CONDITIONS IN MARYLAND, AS RELATED TO PLANT GROWTH
}

\author{
By \\ FORMAN T. MCLEAN \\ Dissertation submitted to the Board of University \\ Studies of the Johns Hopkins University in \\ conformity with the requirements \\ for the degree of Doctor of \\ Philosophy
}


s 


\title{
A PRELIMINARY STUDY OF CLIMATIC CONDITIONS IN MARYLAND, AS RELATED TO PLANT GROWTH
}

\author{
$\mathrm{By}$ \\ FORMAN T. MCLEAN \\ Dissertation submitted to the Board of University \\ St udies of the Johns Hopkins Lniversity in \\ conformity with the requirements \\ for the degree of Doctor of \\ Philosophy
}




$$
\begin{aligned}
& S 600 \\
& . M 4
\end{aligned}
$$




\title{
A PRELIMINARY STUDY OF CLIMATIC CONDITIONS IN MARY- LAND, AS RELATED TO PLANT GROWTH ${ }^{1}$
}

\author{
(Carried out under the auspices of the Maryland State \\ Weather Service, in 1914)
}

\author{
FORMAN T. MCLEAN
}

\begin{abstract}
${ }^{2}$
This study is an attempt to test certain methods for determining some of the quantitative relations between climatic conditions and the growth of plants. Since these relations are very complex, and since the interpretations of experimental results bearing on these relations are exceedingly difficult, the preliminary stages of such interpretations may be advanced by employing the growth rates of a standard plant as a measure of the effectiveness of the surroundings to produce growth. To do this, it is necessary to employ plants that are as nearly alike as possible at the beginning of the various tests. The plant is thus regarded as a sort of integrating and recording instrument, the reading of which is zero at the beginning of each observation period. The plant is allowed to grow during the period, and the effectiveness of the environmental conditions during that time is measured in terms of the amount of growth produced.

This method was employed in these studies, the plant used being soybean. A new observation period began approximately every two weeks and continued for a month, so that the different periods overlapped. Observations on growth were also made at the end of about two weeks; that is, at the middle of the month.

To have the plants of all tests nearly alike at the beginning of the period, they were always started from the seed. Dry seeds change less rapidly with time and are less influenced by surrounding conditions than plants in any other developmental phase. The growth here studied is thus that occurring during the first two weeks and during the first month, from the seed.

At the beginning of each period, seeds were planted in plunged pots, the same soil being used for all stations, and the pots were furnished with auto-

\footnotetext{
1 Botanical contribution from the Johns Hopkins University, No.47.- In the editing of this paper I have been assisted by Mr. F. M. Hildebrandt.-B. E. L.

2 The manuscript of this paper was received July 1, 1916. This abstract was preprinted, without change, from these types, and was issued as Physiological Researches Preliminary Abstructs, vol. 2, no. 4, January, 1917.
} 
irrigators, to prevent the cultures from ever suffering from lack of soil moisture. The influence of rainfall, as it affects soil-moisture, was therefore removed from the main consideration. Temperature, evaporation and sunshine are thus the climatic conditions with which the study mainly deals.

Only two stations are here considered, Oakland (in the mountains of western Maryland) and Easton on the (eastern shore of Chesapeake Bay). Evaporation was measured by means of standardized cylindrical porouscup atmometers. Daily maximum and minimum temperatures were obtained in the usual manner. Sunshine records are considered to some extent, as are also those of rainfall and soil-moisture.

After about two weeks of growth from the seed, the following growth measurements were recorded: stem height, average number of leaves per plant, average length and width of mature leaves, and average of the products obtained by multiplying length by width for each leaf. After about a month of growth, these measurements were repeated and, also, the average leaf area and the average dry weight of tops per plant were determined.

Apart from the study of various methods for growing and measuring the plants, measuring the climatic conditions and interpreting the data thus obtained, the present studies also yield some very definite indications regarding the interrelations holding between the various climatic features, on the one hand, and the manner and rate of development of the plants, on the other. These results are summarized below.

1. Considering the entire period of observation at each of the two stations here employed (which embraced nearly the entire frostless season at each station), the complex of environmental conditions experienced at the Easton station was much more efficient in producing growth of soy-bean plants than was the corresponding envirommental complex experienced at Oakland. The three criteria mainly used in these studies for measuring plant growth-leaf area, stem height and dry yield of tops-all agreed in pointing to this conchusion. For the first two weeks of growth from the seed, the average daily growth increment in terms of leaf-product (the mean of the products obtained by multiplying the length by the breadth of each leaf) was 1.2 for the Easton season, and 0.9 for the Oakland season. The length of the season employed at Easton was 171 days, while the growing season began later and was terminated earlier at Oakland, where the length of season actually employed was 103 days. The total efficiency of the Easton season to produce plant growth, as in these tests, may therefore be regarded as proportional to $171 \times 1.2$, or 205.2 , and the efficiency of the Oakland season may similarly be taken as proportional to $103 \times 0.9$, or 92.7 . The total efficiency of the Easton season of observation (its power to produce plant growth) thus appears to have been 2.21 times as great as was the corresponding efficiency of the Oakland season. About one month of the actual frostless scason at Faston was not included in the season of 
these studies, however, so that the total efficiency of the Easton frostless season for 1914, measured in terms of leaf-product as here used, was about 2.5 times as great as that of the Oakland frostless season.

2. Of the five criteria by which the growth rates of the experimental plants were compared (stem height, total number of leaves, produced, leaf dimensions, leaf surface and dry weight), those of stem height, leaf surface and dry weight exhibited the greatest differences between different eulture periods. The rates of growth in terms of leaf surface and in terms of dry weight varied in a similar manner with the same kind of variations in external conditions, while the growth rates measured in terms of stem elongation varied in another way with the same external differences. It thus appears that the rate of elongation of plant stems is influenced by external conditions differently from the rates of development of leaf surface and of dry weight for the same plants. In dealing with the quantitative relations of plant growth to external eonditions it is therefore necessary to distinguish clearly between the various kinds of growth and the various criteria that may be employed in their measurement.

3. The rates of growth in stem height were generally more rapid during the first than during the second fortnight of growth from the seed, for both stations. On the other hand, the rates of increase in leaf area (as approximately measured by means of the leaf-product) were generally more rapid during the second fortuight.

4. The growth rates generally showed very evident seasonal marches, by whatever criterion they were measured, increasing during the first part of the season and decreasing in the autumn. These seasonal marehes were most apparent for the first two weeks of growth from the seed, and were most clearly shown by the rate of inerease in stem height. They correspond, in general trend, to the seasonal marehes of the temperature conditions.

5. The seasonal marches of both the growtl rates and the temperature values for Oakland are quite markedly different from those for Easton. Both ranges are greater for Easton than for Oakland. The highest temperature values and the highest growth rates oceurred at Easton, and the growing season was terminated by killing frost earlier at Oakland than at Easton. Nevertheless, the last two-week period before autumn frost at Oakland exhibited a higher temperature value and higher growth rates than did the last two-week period before frost at Easton. This difference between the magnitudes of the final minimum growth rates observed at the two stations appears to emplasize one of the main differenees between a mild, equable, coastal elimate and a much more rigorous mountain climate, as these may influence plant growth. In the milder climate of Easton, with its small daily range of temperature, the frostless season is apt to be prolonged until the growth of many plants is much reduced or entirely checked by low temperature. In the mountain elimate of Oakland, however, with its large 
daily range of temperature and high nocturnal radiation, very low night temperatures and frosts occur earlier in the season, while the day temperatures and the growth rates of many plants are still high. These differences between the two stations, as regards the temperatures and growth rates exhibited at the close of the season (just before autumn frost), are surely intimately associated with the two types of climate here illustrated, and are of undoubted importance in the consideration of plant life in general. Another difference to be noted between the two stations here considered refers to the time of occurrence, within the growing season, of the maxima of temperature and of growth rates. These.maxima occurred about a month earlier at Oakland than at Easton-a fact that may be of significance in the comparative seasonal climatology of these stations, at least for the summer of 1914.

6. The mean rate of leaf enlargement (as measured by the leaf-product) and also the mean rate of increase in dry weight, for the four-week periods of growth, followed seasonal marches that showed a secondary influence of the moisture conditions of the surroundings, as well as the primary one exerted by temperature. No apparent relation exists, however, in the data of the present study, between the growth rates, on the one hand, and the data of either rainfall or evaporation, on the other; perhaps because the culture plants were protected from soil drought by auto-irrigation. The general moisture conditions of the surroundings were measured in terms of the ratio of rainfall to evaporation, however, and it is with reference to this ratio that the above-mentioned secondary influence of these conditions becomes apparent. This moisture influence appears to be most clearly shown by the growth rates for periods when the daily mean temperature was high $\left(66^{\circ}\right.$ to $76^{\circ} \mathrm{F}$.). Apparently it is the moisture conditions of the second half of the four-week period that are here influential. At the end of a month of growth from the seed the mature leaves are larger when the last two weeks of the period have been characterized by a high value of the rainfall-evaporation ratio than when these two weeks have been drier. This is related to the fact that the leaf development of the first month of growth mainly occurs in the latter half of the period.

7. It appears that temperature was clearly the limiting condition (in the usual sense) for growth during the first two weeks, in practically all cases. During the second two weeks of growth, however, with exactly the same envirommental conditions, the moisture relation (rainfall-evaporation ratio) appears in many eases to have been the limiting condition for growth, this heing especially true, as has been remarked just above, when the temperature was high. It thus appear's that if two plants in clifferent stages or phases of their development are exposed to the same fluctuations in envirommental conditions, the limiting condition for one plant during a suceeding period may he of an entirely different nature from that for the other. This must 
be due to a difference between the internal conditions of the plants at different developmental stages. While this principle is so obvious as to appear not to require emphasis, it seems seldom to have been seriously considered in the literature of ecology and physiology. It must be considered wherever standard plants are employed for the comparison of climates

\section{INTRODUCTION}

\section{THE GENERAL PROBLEM}

The dependence of plants upon climatic conditions is almost self-evident, but the quantitative aspect of the relation between plant activities and climate presents an exceedingly complex problem, the solution of which can not be expected for a very long time. Many investigators have attacked this problem, attempting to measure plant production or crop yield in terms of the climatic conditions observed to be present during the growth period. This sort of research has usually resolved itself into attempts to correlate plant growth with one, or at most two, climatic factors-generally with temperature and rainfall, since these are both subject to marked geographical and temporal variations, and since both produce very evident effects upon the manner of growth of plants. The influence of temperature upon plant growth is marked and easily observed. Rainfall affects plant growth mainly in an indirect way, through its influence upon soil moisture. A number of other factors, however, both climatic and non-climatic in character, are also continually exerting infuences on plants, and plant growth is an expression of the effects of all these influences combined. Among these other, less frequently mentioned factors are: quality and intensity of sunlight, the evaporating power of the air, wind velocity, presence or absence of parasitic organisms, and many others.

With so many variable factors entering into the equation that may be thought of as expressing the complex set of relations here suggested, no precise correlation between plant growth and any single factor is to be expected. Nevertheless, temperature or rainfall does sometimes act as the most important variable factor, producing the greatest variations in plant growth from year to year or from season to season in any giren region or locality, as has been shown by several workers. Surprisingly close agreements were found by Arctowski ${ }^{3}$ and by Smith, ${ }^{4}$ between crop yield and amount of rainfall, and Merriam ${ }^{5}$ found a distinct general correlation between normal temperature conditions and the present distribution of plant and animal

\footnotetext{
3 Arctowski, IIenryk, Studies on climate and crops: corn crops of the Lnited States. Bull. Amer. Geog. Soc. 44: 745-760. 1912 .

4 imith, J. Warren, The effect of weather upon the yicld of corn. Monthly Weather Rev. 42: 7S-92. 1914

s Merriam, C. Hart, Laws of temperature control of the geographic distribution of plants and animals. Na. tional Geog. Mlag. 6: 229-23s. 1594.
} 
life on the Pacific coast of the United States. It does not appear, however, that such results are to be generally expected. A large number of factors are constantly varying in nature, and many of these are undoubtedly effective to produce variations in the manner and rate of growth of plants. One single factor may be most influential, as rainfall in desert regions generally, but very many other conditions are also important for plant growth, and alterations in any of these effective conditions must surely exert some influence on the rates of the physiological processes in plants subjected to such alterations. ${ }^{5 a}$

Not only do external conditions about the plants change, but the plants themselves also change as time goes on; they respond differently to the same external influences at different times in their life cycles or in different stages of their development. It follows that plant growth cannot be capable of expression in terms of climatic or other cxternal factors, excepting by means of an exceedingly complex formula, which should involve all of the effective or controlling conditions. We are probably not yet even acquainted with all the factors that influence plants, nor do we know the action of those with which we are acquainted, so that attempts to establish what might be called a complete envirommental formula,-representing the total effectiveness of the surroundings to produce growth, maturation of seed, etc., for any given plant form,- - must be postponed for a long time.

The rate of growth of any given plant, however, is itself an expression of the sum total of all the effects of all the external conditions as these acted during the period of measurement. ${ }^{6}$ Consequently, if it were possible to grow standard plants in different enviromments, it should be feasible to measure and compare these enviromments in terms of their capacities to produce growth in the standard plants.

Of course, such a procedure as that here suggested can be of but relatively little value in the interpretation of crop production, etc., unless the environmental conditions may be assorted into several groups which may be separately studied. To study them in this way involves the problem of maintaining certain groups of conditions sensibly alike for different standard plants, while other groups are varied. Thus the soil conditions, taken as a group, may be similar for a number of plant cultures, while the atmospheric conditions may be different. Differences in growth, etc., may then be considered as due to the influence of the atmospheric complex, acting with the internal conditions that make up the nature of the plants used.

5a While the present paper was in press there appeared the following very important report of a physical study of the relation of plant transpiration to certain environmental conditions:-

Briggs, L. J., and H. L. Shantz, Daily transpiration during the normal growth period and its correlation with the weather. Jour. Agric. Res. 7: 155-212. 1916. See also: Kiesselbach, T. A., Transpiration as a factor in crop production. Nebraska Agric. Exp. Sta. Research Bull. 6. 1916.

${ }^{6}$ Livingston, B. E., Climatic areas of the United States as related to plant growth. Proc. Amer. Phil. Soc. 52: 257-275. 1913. See especially page 258 . 
This method of study should be valuable, of course, only when it may be assumed that the standard plants were alike at the beginning of the period of measurement. If they were not sensibly alike, interpretation of the observed differences in growth becomes practically impossible, for in such a case the argument is hopelessly complicated by the fact that the different intermal-conditions initially effective in the various cultures enter into the logieal analysis. To interpret the results obtained with plants that were unlike at the beginning of the experiment, an analysis of the internal conditions would first have to be made, and this presents far more difficulties than does the analysis of the relation between external conditions and the rate of growth.

The mode of attack thus suggested was followed in planning the study here to be reported. ${ }^{7}$

\section{GENERAL PLAN OF STUDY}

The plants. To investigate the influence of climatic conditions upon the growth of standard plants, it thus appears desirable to grow cultures of these plants under the different climatic complexes that are to be considered, and to treat all the cultures alike in all other respects. Such like treatment of the cultures cannot be actually attained as yet, but an approach to this is possible. While it is to be remembered that plants vary greatly among themselves, on account of various conditions as yet not well understood, and that they cannot at present be standardized in the same sense as thermometers and many other physical instruments, nevertheless this kind of study may be expected to elucidate some, at least, of the fundamental relations of plant growth to climatic conditions.

The choice of standard plants for such investigations is rendered difficult by several considerations. Since any given plant individual alters with the progress of time and according to its treatment, it is clear that plants that have been subjected to different treatments before the beginning of any comparative test are not to be employed. The standard plants must be considered as integrating instruments and, ideally, should be set at the "zero points" of their scales when the tests begin. This means that the plants employed must be in a stage of their development such that any differences that may have occurred in their past treatment have been registered in growth, internal change, etc., to as slight a degree as possible. Such a stage is presented in the seed; in this dormant phase the organism is but slightly affected by ordinary environmental variations. The seed was. therefore chosen as representing the zero point of growth in the study be here presented.

7 A short preliminary paper covering certain phases of this stucly las already appeared: MeLean, Forman T., Relation of elimate to plant growth in Maryland. Ionthly Weather Rev. 43: 65-72. 1915. 
The species here employed were soy-bean (Glycine hispida Maximov.), Windsor bean (Vicia faba L:) maize (Zea mais L.) and wheat (Triticum sativum L). Only the results obtained with the first of these species, soybean, will be dealt with in the present paper. In order that the seeds actually used for comparing the different sets of climatic curroundings might be as nearly alike as possible, they were all of the same strain and of the same crop, for each species employed. Furthermore, each lot was sorted, and all that appeared abnormal for any reason were discarded.

The plants were grown from the seed in cultures in which all environmental conditions except those usually regarded as climatic were controlled as fully as was practicable, and their rates of growth were used as a measure of the effectiveness of the whole group of surrounding conditions. The rate of growth is, of course, the amount of growth accomplished in a given unit of time, and it may be measured in terms of the amount of plant produced during the observation period. The quantities measured in this study were the size and weight of the plant produced, beginning with the seed, during periods of about two weeks and of about four weeks after planting. Dividing the result thus obtained by the number of days in the period gives the average daily rate of growth. The average of the rates of growth for several plants was taken as a measure of the comparative effectiveness of the climatic conditions as these tended to produce differences in plant activity at the several stations, during the growth period. These average growth rates were compared to values obtained by instrumental measurements of the climatic factors surrounding the plants during their period of growth.

The cultures were started approximately every two weeks during the growing season, at each of the stations employed, and the growth obtained in each culture was determined after about two weeks and again after about a month.

Measurement of the climatic conditions. No attempt was here made to secure a complete evaluation of the climatic conditions that affect plants. Indeed, such an attempt must be quite futile until much more is known about what climatic factors do effect plant growth, and how they act. The regular observations of the U. S. Weather Bureau, for temperature, rainfall and duration of sunshine, with supplemental data bearing on soil moisture and evaporation, were brought together in various ways, for comparison with the plant growth rates for corresponding periods.

Nine coöperative stations of the U. S. Weather Bureau in Maryland were employed in this study. Their geographical locations are shown on the chart of figure 1. Of four stations on the Coastal plain, three (Easton, Princess Anne, and Coleman) are east of Chesapeake bay, and the remaining one (College Park) is much farther inland, near the borderline between the Coastal plain and the Piedmont plateau. Four stations are on the Piedmont plateau, one (Baltimore) at its lower edge near Chesapeake bay, two 

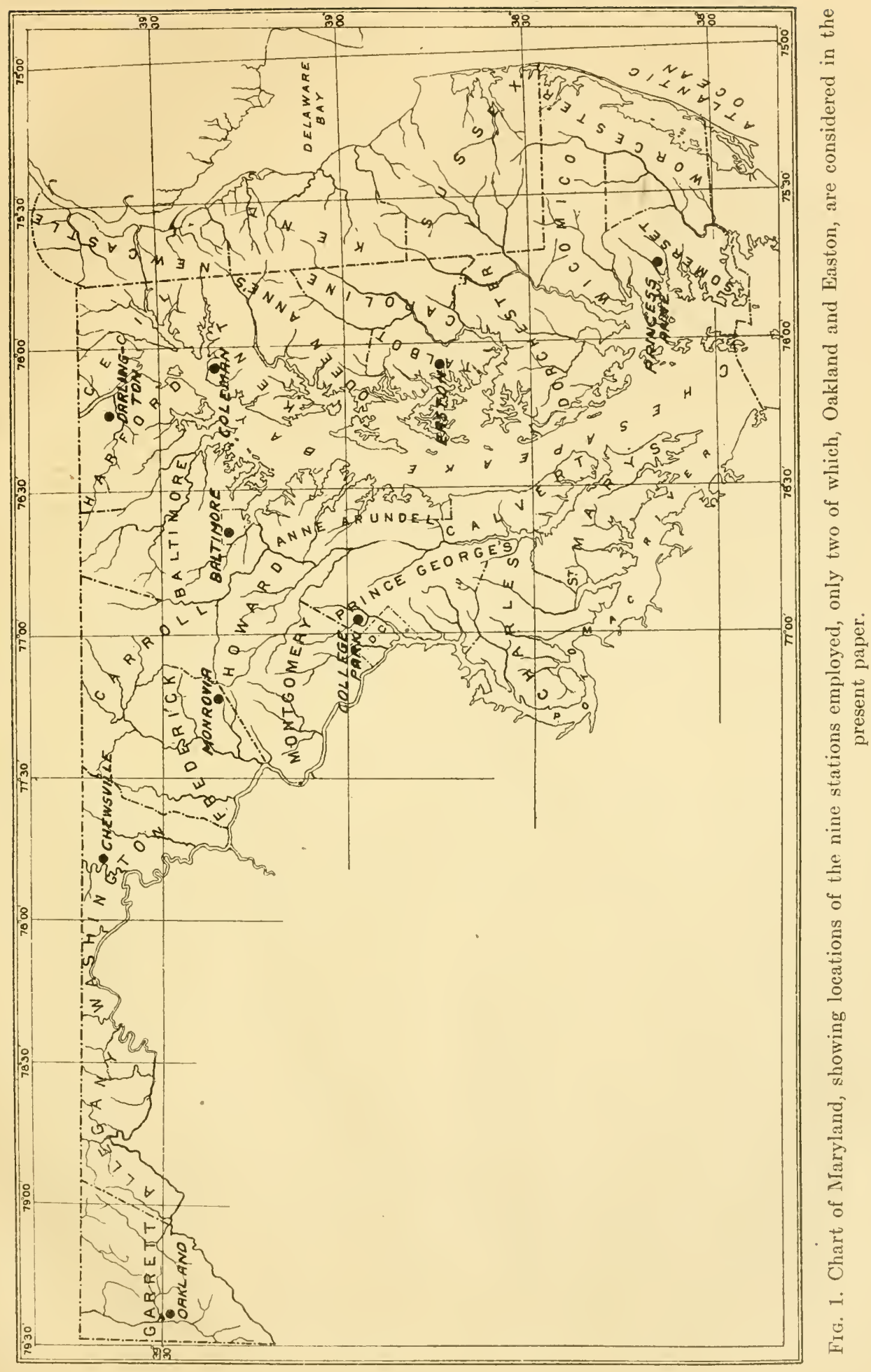

137 
(Darlington and Monrovia), in the hilly country north and west of Baltimore, and one (Chewsville) in the Hagerstown valley. The ninth station (Oakland) is on the tableland at the top of the Allegheny plateau. All of these stations excepting Oakland are at comparatively low elevations, less than 1000 feet above sea level. Oakland has an altitucle of about 2500 feet.

Without the most cordial support of the coöperative weather observers at the various stations this project could not have been successful, and the writer takes pleasure in here expressing his grateful appreciation of their very generous assistance. It is with much regret that mention must here be made of the death of Mr. J. S. Harris, the observer at Coleman. The other observers who coopperated in this work were: Mr. A. F. Galbreath, of Darlington; Mr. J. H. Lawson of Monrovia; Mr. D. P. Oswald, of Chewsville; President H. J. Patterson, of College Park; Mr. H. Shreve, of Easton; Mr. J. R. Stewart, of Princess Anne; and Mr. R. E. Weber, of Oakland.

Of the nine stations only two, Easton and Oakland, will be considered in the present paper, these being chosen to represent extremes of climatic conditions. Easton is on the flat coastal plain and has the characteristic humid, equable climate of the eastern shore of Chesapeake bay. It has an altitude of only 63 feet above sea level, The climate of Oakland, on the other hand, is typical of the moist but variable climate of the Allegheny plateau. The latter station lies on a gentle south slope, at an altitude of about 2500 feet.

\section{ACKNOTLEDGEMENTS}

The present studies were carried out under the auspices of the Maryland State Weather Service, and those of the Laboratory of Plant Physiology of the Johns Hopkins University, during the summer of 1914. The study was under the general direction of Prof. B. E. Livingston, to whom the author wishes here to express his great indebtedness for advice and aid, which alone made the work possible. The writer wishes also to acknowledge his indebtedness to Prof. W. B. Clark and to the other members of the Board of Governors of the Maryland State Weather Serivee, for their esteemed support; to Dr. Oliver L. Fassig, Director of the Maryland Division of the U.S. Weather Bureau, for assistance in securing and compiling the weather data here used and in the selection of the stations at which the work was done; and to his associates, in the general project, Miss A. Hopping, Dr. J. W. Shive and Mr. E. S. Johnston, for coöperation and assistance. He also wishes to express his thanks to Mr. J. Calvert, who most kindly allowed the soil used in this study to be taken from his property, and to Prof. IV.T. L. Taliaferro and to Mr. Grover Kinsey, who arranged for and supervised the shipment of the soil to the various stations. 


\section{METHODS AND EXPERIMENTATION.}

\section{THE PLANTS}

The seeds and their treatment. The soy-bean used in this study was of the variety called "Peking," with small black seeds. The plants are rather small but erect growers. The seed was of pure strain, obtained from the 1913 erop of the Maryland Agricultural Experiment Station. Tests of it showed 98 per cent. of germination, when planted in moist quartz sand, in earthen pots in the greenhouse, at a temperature of about $58^{\circ}$ to $71^{\circ} \mathrm{F}$, during the early part of Mareh, 1914. The seeds appeared very uniform. All small and all unusually large seeds, as well as any that appeared otherwise abnormal, were discarded.

All the seeds were treated with carbon bisulphicle vapor for one week, to destroy insects. Immediately after treatment, on March 27, 1914, the seeds were transferred to paraffined paper eylinders holding a quart, with tight-fitting covers. They were then stored in a laboratory locker, with a rather uniform temperature of about $72^{\circ} \mathrm{F}$, until taken into the field for use.

The seeds were planted $2.5 \mathrm{~cm}$. ( 1 in.) deep, in moist soil in $15 \mathrm{~cm}$. (6 in.) flower pots of the usual conical form, six seeds being planted in each pot. No records were kept of the dates of appearance of the seedlings above the soil at either of the two stations here considered, but such records are available for three of the other stations, Chewsville, Darlington and Monrovia. The average time required for the plants of soy-bean to appear above the ground at these three stations was 5.5 days from the date of planting. In the majority of eases this time was from 4 to 6 days, the extremes recorded being 3 and 11 days. The slow rates of germination oceurred during cold periods. The rapidity of germination will not be considered in the discussions that follow; the date of planting appears to furnish a more satisfactory initial point for calculating the growth rate than would that of the appearance of the seedlings above the soil.

The growth measurements. Growth may be considered as essentially the process of development toward mature size, and it may be measured in several ways. In this investigation the growth rate of the soy-bean plants was considered from four points of view: (1) the rate of elongation of the plant shoot, (2) the rate of production of leaves, (3) the rate of development of leaves [in terms (a) of linear dimensions, and (b) of superficial area], and (4) the rate of increase in the dry weight of the plant. It is important that the measurements made in securing this sort of data be as rigidly uniform as possible, to render the results comparable, and a regular method and order of measurement were therefore instituted and adhered to throughout the season.

Each station was visited at intervals of approximately two weeks. At the time of the first visit after planting, the height of the stem, and the length 
and greatest width of each leaf or leaflet were separately recorded for each plant. The stem height was measured from the soil surface to the base of the terminal bud; the base of the bud was here employed, rather than of the extreme tip, because the length of the bud is affected by the stage of development of the youngest visible leaf, and is therefore variable. The leaves were measured in regular order, from the base of the plant upward, each one being given a serial number in this order in the records. The leaf lengths were measured from the junction of blade with petiole to the tip of the blade. The width was measured at the widest part, and at right angles to the longitudinal axis of the blade. All linear measurements were made to the nearest millimeter.

Approximately four weeks after planting all the measurements just mentioned were repeated, after which the plants were cut off at the level of the soil. The leaves were immediately placed in a photographic printing frame and sun prints were made on photographic paper, from which the extent of the total leaf surface was later obtained, by means of a planimeter. Any plants that appeared to be unusual, because of accidental injury, and those that were markedly smaller or larger than the average were discarded. The plants of each culture were mailed to the laboratory at Baltimore, where they were dried for several days in the greenhouse, and then desiccated in an oven at $100^{\circ} \mathrm{C}$, , to constant weight. The dry weights were determined to 0.01 gram. These original dry weights included the cotyledons, when present, but these do not properly constitute a part of the growth of the plant after germination, and so the dry weights were afterwards corrected by determing the weight of the cotyledons in each case and subtracting this from the original amount recorded. This correction has been applied to all of the dry weight data employed in the present paper.

As has been indicated, one culture of each of the four species used was started approximately every two weeks, at each station, and each culture was measured as described above. The results of these numerous measurements for soy-bean are summarized in tables I to IV, tables I and III referring to the measurements of the plants when about two weeks old from seed, and tables II and IV referring to the second (and final) measurements of the plants, when they were about four weeks old from seed. These tables are similar in form, so that an explanation of tables I and II, for Oakland, will also serve to describe the manner of presentation of tables III and IV, for Easton.

In table I the first four lines give general data. The different cultures are numbered in chronological order in the first line. The "date of planting" is given in line 2. "Number of plants" (line 3) refers to the number actually used in the measurements and thus excludes abnormal individuals which were discarded.

The data of lines 5 to 13 present a summary of the plant measurements 
obtained about two weeks after planting. "Age" (line 5) indicates the length of the period, in days, from the date of planting to the date of observation, which was only approximately two weeks, the variations in this respect being due to the exigencies of the many trips necessary to each of the nine stations that were under observation. In reckoning these ages the date of planting was not included, the period beginning with the day after that date and ending with the date of observation. This age varies from 9 days in the case of eulture 1 at Oakland to 17 days for culture 1 at Easton (table III). These differences necessitate that all of the data be ultimately expressed in the form of mean daily rates, or averages, in order that comparison of the different cultures may be possible. In all the plant measurements here given the data are averages per plant, the number of plants from which these averages are derived being given in the third line. Thus, the recorded stem height (line 6$)$ for culture $1(2.4 \mathrm{~cm}$.) is the average height of the four plants of that culture, being expressed with the same degree of aceuracy as in the case of the original measurements. The average daily increase in stem height (line 7 ) is obtained by dividing the average stem height (line 6) by the corresponding number of days (line 5), in each case. Line 8 shows the average number of full-grown leaves per plant, this number being obtained by summing the number of leaves that had been developed, whether these were still present at the time of measurement or had previously died and fallen. Leaves that were approximately half-grown were considered as half leaves. The purpose of this enumeration is to get an expression of the stage of development of the plants; that is, to show how far the plants had progressed in their life eycle. Thus, a plant with three leaves mature and one half-grown is recorded as more advanced in growth than a plant with only three leaves, but appears as less advanced than a plant with the fourth leaf fully developed. Line 9 gives the average daily increment in the average number of leaves per plant, these data being obtained by dividing each number in line 8 by the corresponding number of days (line 5).

The average leaf dimensions ( $l$ and $w$ ), given in lines 10 and 11 , serve for comparison of the relative sizes of mature leaves in the different cultures. The unit of enumeration here employed was not the same as in the case of the number of leaves present (line 8 ). For the data of line 8 all leaves, whether mature or only partly grown, were considered, and the whole leaf, whether simple or compound, was taken as a unit; for the leaf dimensions $l$ and $w$, on the other hand, only mature leaves were measured (or those very nearly mature, for small plants), and here the unit is a leaf or a leaflet, as the case may be. It is of no serious moment for the present purpose, whether the unit of surface is a leaflet of a compound leaf (as the secondary, alternate leaves of soy-bean) or a simple leaf (as each of the initial pair of opposite leaves). The average "leaf-product," $P$ (line 12), is the average per plant of the sum of the products of length multiplied by width for all 
leaves, whether young or mature. These average leaf-products serve for general comparison of the relative leaf areas of the plants in the different cultures. The average daily increase in leaf-product (line 13) is obtained by dividing the average leaf-product (line 12) by the corresponding number of days (line 5). The derivation of the soil moisture percentage (line 14) will be fully explained in connection with the discussion of soil environment, so that it will suffice to state here that each figure given is the average of the soil moisture data obtained for the culture in question during the period covered by the corresponding plant measurements.

The datagiven in table II, also for Oakland, are the final measurements of the plants at the time of harvest, when they were approximately four weeks old. The data given in lines 1 to 13 and in line 19 correspond to the similar data of table I, lines 1 to 14 , and need no further comment here. Lines 14 to 18 , however, present data not considered in table I. "Leaf area" (line 14) is the average total area per plant of either leaf surface, the lower or the upper, but not of the total leaf surface, which would be double the value given. This leaf area $(A)$ was obtained by means of a planimeter, from photographic prints of the leaves, as has been mentioned. The average daily increment of leaf area (line 15) is obtained by dividing each number in line 14 by the corresponding number of days (line 5). The average dry weight (line 16) is the average weight per plant of only the top portion, excluding the parts beneath the soil surface, as has also been stated. The average daily increment in dry weight (line 17) is the quotient of the average dry weight (line 16) divided by the corresponding age (line 5). The ratio of the average leaf-product to the average leaf area $\left(\frac{P}{A}\right)$ is given in line 18. This will receive attention later.

A ppearance of plants. Tahles I to IV give the measurements of the plants grown in the different cultures at the two stations, but they give no information about the manner of development of the plants or about the possible variable influences that may have affected them and that were neither measured nor controlled. It is desirable to consider here the general thrift and observed development of the plants, as well as some of the variable non-climatic factors that may have influenced the cultures.

At the tine of the first observations, about two weeks after planting, the cotyledons, which were still green and in healthy condition, were always still attached to the young seedlings. The leaves were also bright green and appeared thrifty. During the first two weeks, then, the cotyledons heing still attached, it may be supposed that the seedlings were subsisting upon the stored nutriment of the seeds, at least in part.

At the time of the final observations, on the other hand, these conditions were different, but variable. At Oakland where the plants had grown rather slowly, the cotyledons were still green, still attached, and probably 
Climatic Conditions in Maryland

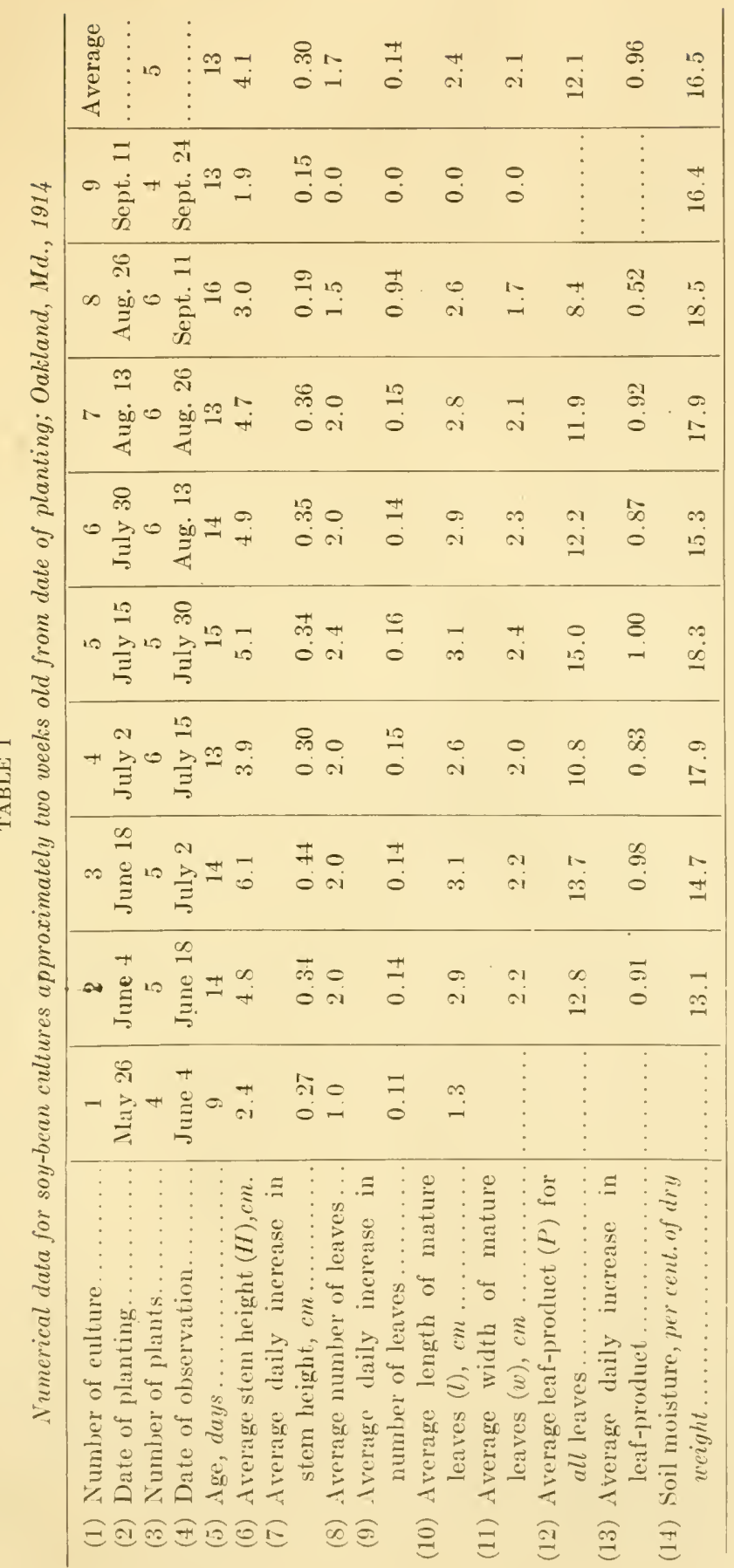




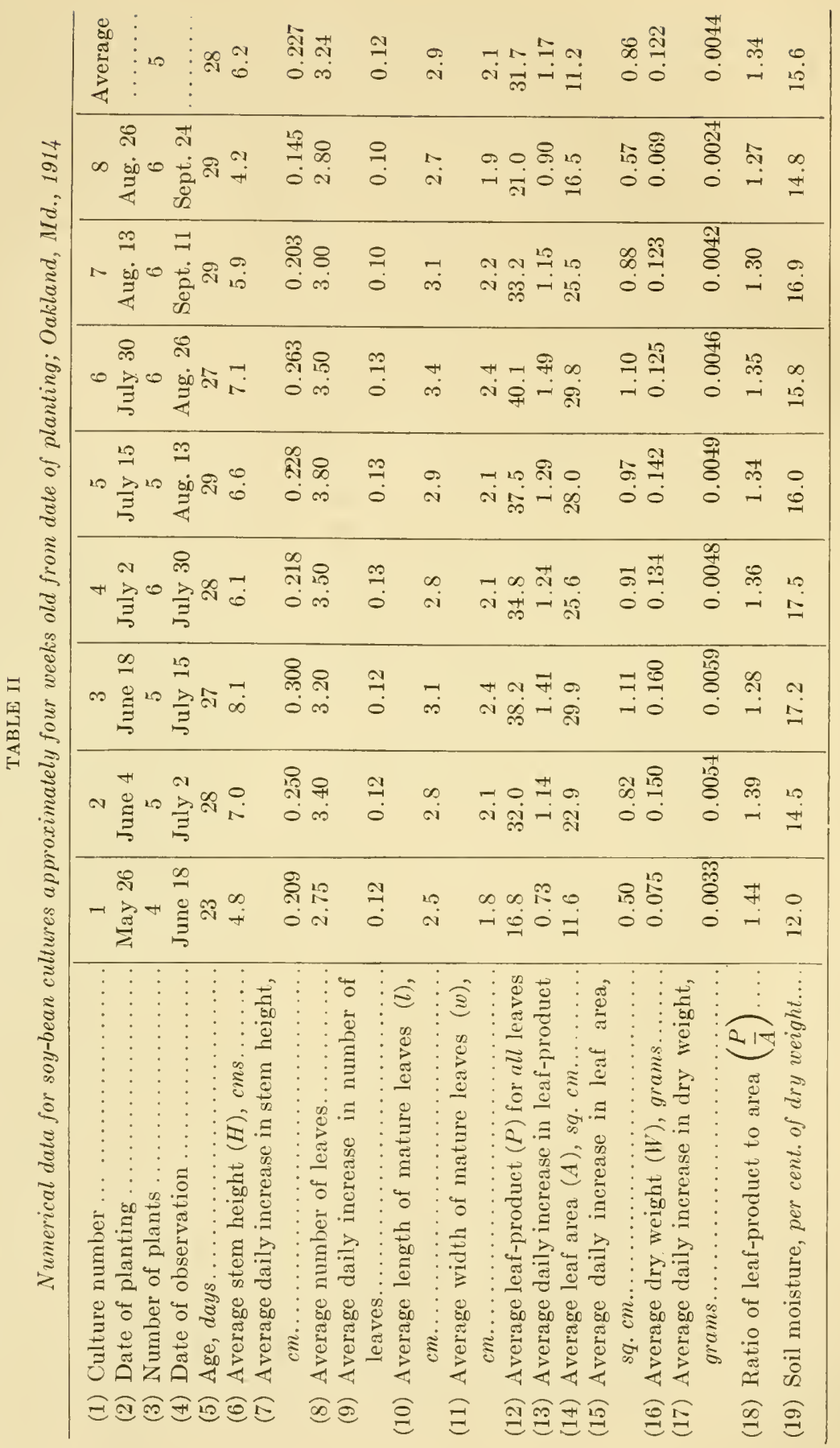


Climatic Conditions in Maryland

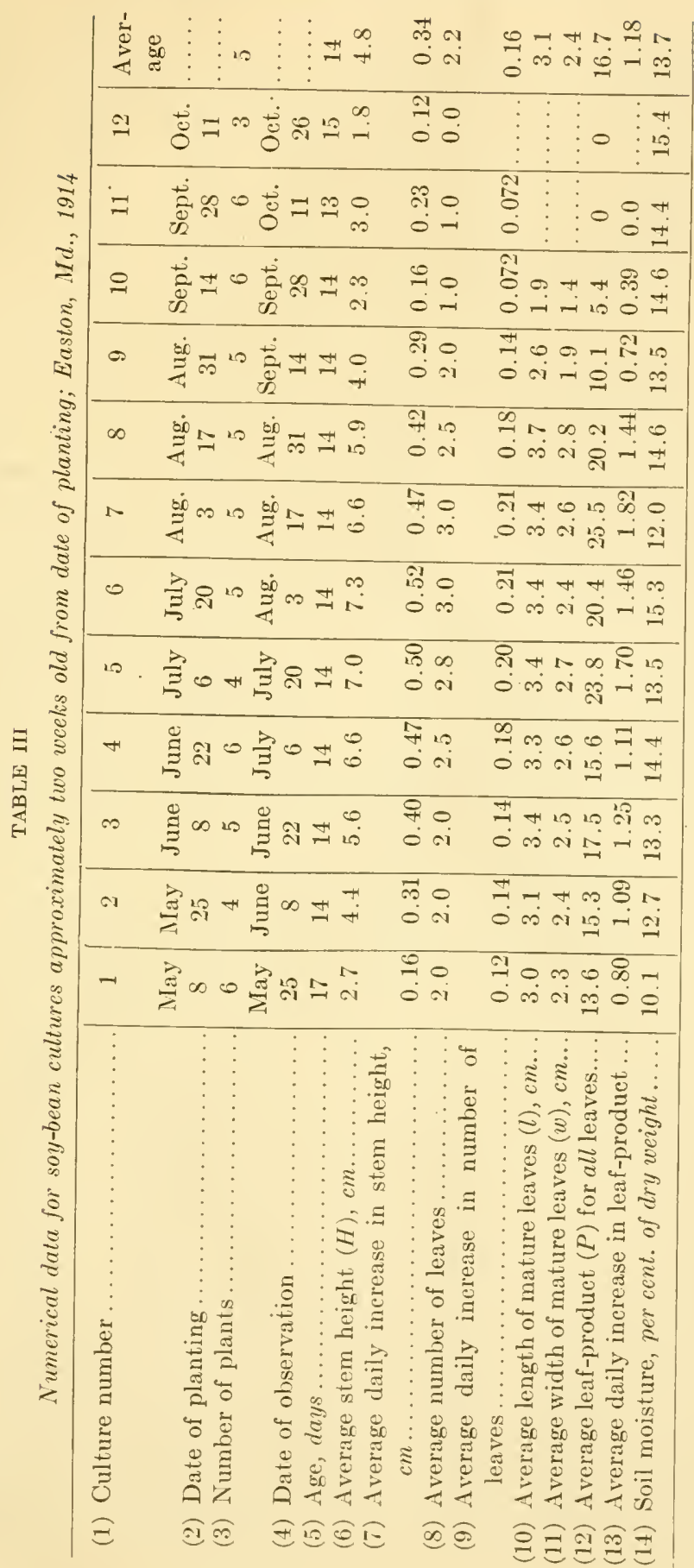

PHYSIOLOGICAI, RESF.ARCHES, VOL. 2, No. 4,

SERIAL NO. I4, FEBRUARY, 1917 


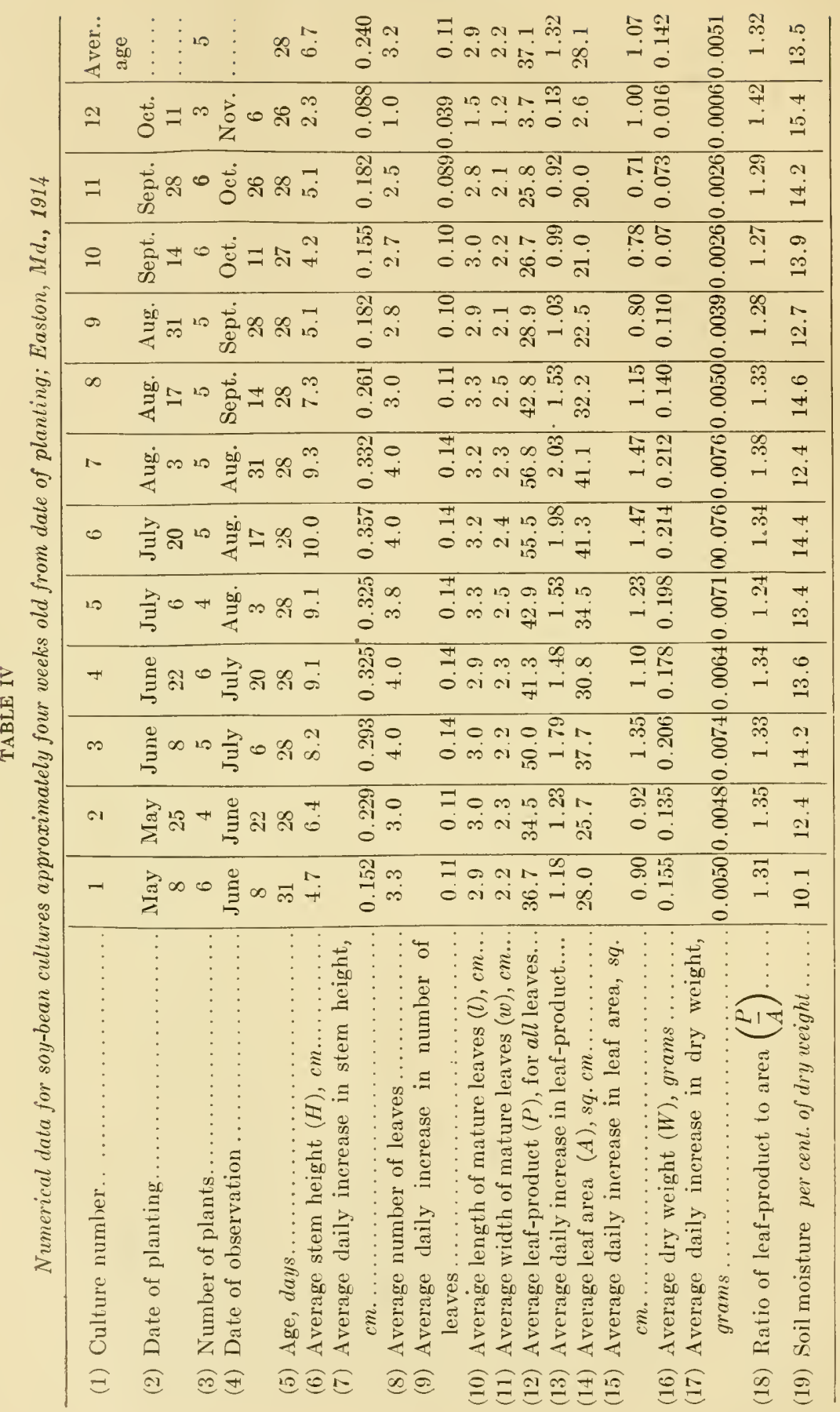


still furnishing material to the growing plants. At Easton, however, this state of affairs was the exception; it was encountered only toward the end of the season, when the plants had grown slowly. In most of the Easton cultures the majority of the plants had either lost their cotyledons, or the latter had turned yellow and were about to fall. Thus, these Easton plants were probably generally independent of the stored food supply of the seeds, at the time of the final observation. Furthermore, these plants were not as healthy in appearance as those in the corresponding stage at Oakland, their leaves being yellowish in color.

It is possible that this unhealthy appearance of the largest and most mature plants at Easton may have been due to the absence of nutrifying bacteria on their roots. The soil used in these experiments had previously supported, among other plants, a wild vetch and is thus to be considered as provided with at least some nodule-forming bacteria, but these may not have been of the right kind for soy-bean. Root nodules appeared in the cultures of Windsor bean but not in those of soy-bean. The absence of root nodules in case of soy-bean, while it almost surely affected the growth rates of the plants, was not a variable factor, as this condition held throughout all of the cultures, and hence it should not interfere with the value of these cultures in comparing the effects of climatic conditions. No other apparently important non-climatic variable factors, which might seriously have affected the growth rates, were noted in the case of these soybean cultures.

\section{THE ENVIRONMENTAL CONDITIONS}

\section{EXPOSURE OF INSTRUMENTS AND PLANTS}

Local and instrumental exposure. The presence of trees or other objects in the near vicinity of the growing plants or of the climatological instruments may seriously influence such environmental conditions as the evaporating power of the air, the intensity of sunshine, wind movement, etc., for the particular exposure in question. It is therefore desirable to have the plants and the instruments located near together, and exposed as similarly as possible so that the growth of the plants and the readings of the instruments may refer to similar atmospheric conditions. It is also desirable that the culture and instrument locations be as much in the open as possible, in order that they mav represent the general conditions of the region as a whole and in order that the data obtained at the different stations may be studied in connection with the regional climatic conditions.

The weather stations for hoth Oakland and baston are situated in the open country. The Oakland station is on a south slope, about $1.6 \mathrm{~km}$. (1 mi.) east of the town of Oakland, and near a public road, which is some- 
what dusty in dry weather. The general nature of the exposure of the plant cultures and instruments at Oakland is shown in figure 2. The cultures, the rain gage and the thermometer shelter were situated near together, about $12 \mathrm{~m}$. (nearly $40 \mathrm{ft}$.) west of the greenhouses of H. Weber's Sons, and were fully exposed on all sides. The greenhouses, being only about $4.5 \mathrm{~m}$. (15 ft.) high, did not produce serious wind obstruction, and they did not influence the light conditions appreciably.

The Easton station was north of the residence of the observer, Mr. Henry Shreve, the general surroundings of the various instruments being shown in figure 3. The plant cultures were $1.2 \mathrm{~m}$. ( $4 \mathrm{ft}$.) north of the rain gage and $18.3 \mathrm{~m}$. (60 ft.) northwest of the dwelling. This position was protected

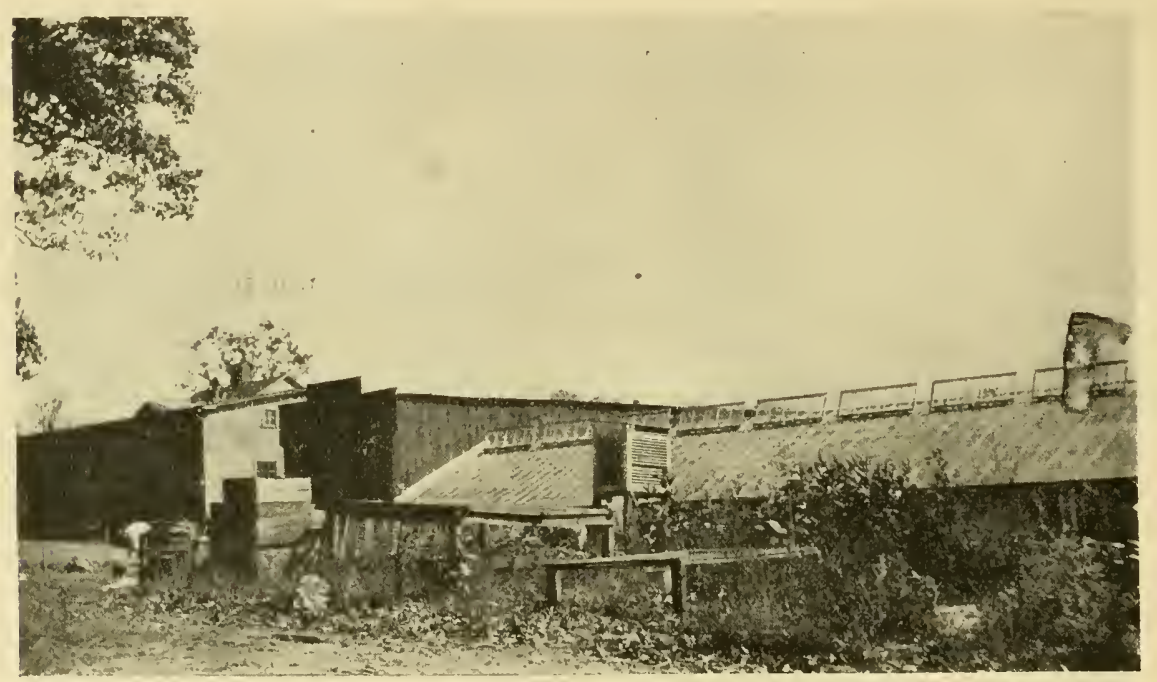

FIG. 2. Culture enelosure and its general surroundings, at Oakland.

on the south, southwest and west by large apple trees, about $15 \mathrm{~m}$. (nearly $50 \mathrm{ft}$.) distant, and by a low shed about $10 \mathrm{~m}$. (33 ft.) distant on the north. The exposure is open toward the east. The crowns of the trees are too high to obstruct wind movement near the ground in a serious manner, but the plant cultures were shaded by the trees from about $3: 30$ until sunset, in June, and from 1 o'elock in September. The thermometer shelter stands under a tree west of the culture location and is about $6 \mathrm{~m}$. (20 ft. $)$ north of the house. Thus, all of the instruments at Easton as well as the plants, were somewhat protected, but not entirely screened, from strong winds from the north, west and south, and the plants were shaded in the late afternoon.

The most pronounced difference between the exposure at Oakland and 
that at Easton lies in the amount of sunlight received. The Oakland plot was exposed to full sunlight throughout the day, while the Easton plot was shaded in the late afternoon.

The climatological instruments (rain gage and thermometers) are of the standard pattern employed by the U. S. Weather Bureau, and have the standard exposure. The thermometers are in the usual shelter, $1.5 \mathrm{~m}$. (5 ft.) above the ground, and thus do not experience all the temperature changes to which are subjected young growing plants near the soil surface. The air temperature around such plants is often greatly influenced by radiation

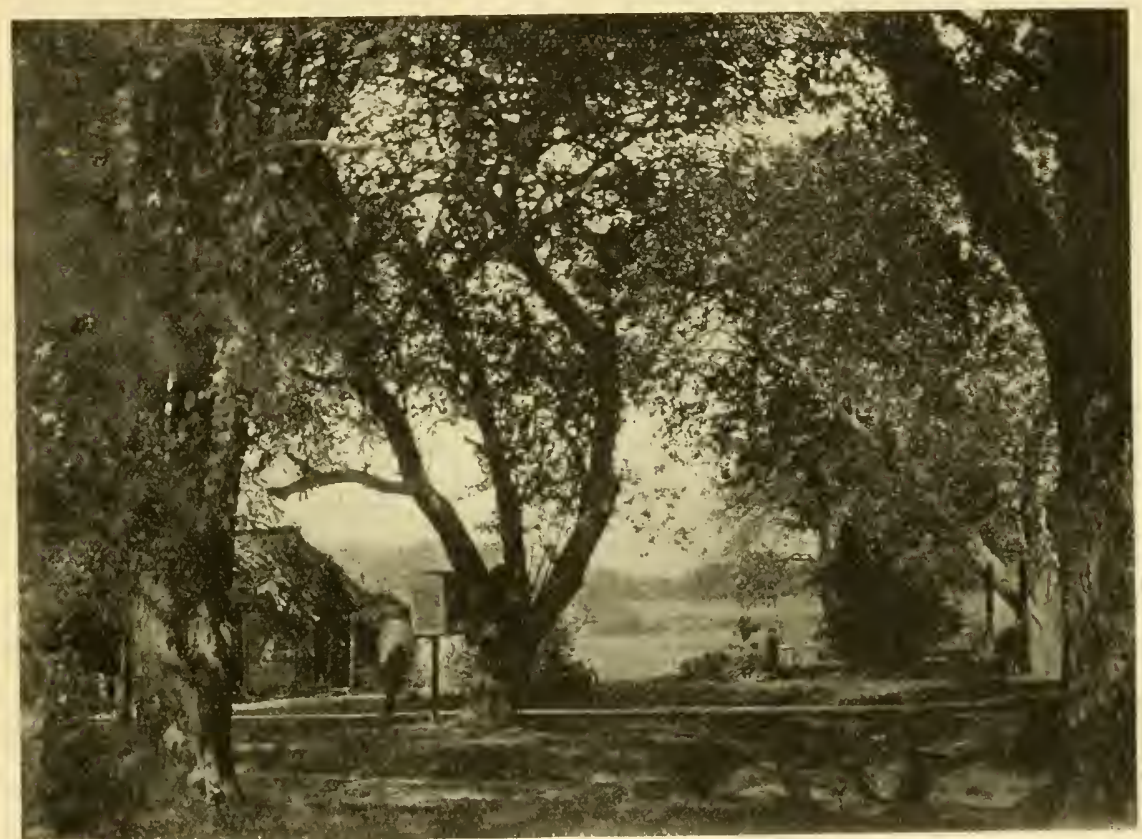

FIG. 3. Culture enclosure (partly shown to the left of the rain gage) and its general surroundings, at Easton.

from the soil, while thermometers with the standard exposure of the U. S. Weather Bureau are much less influenced in this way. The warming of the earth by isolation, and the rapicl cooling on clear nights, by radiation into the atmosphere, subject low plants to extremes of temperature not usually recorded by the thermometers. It seems fair to suppose, however, that there is probably a fairly constant relation between the average daily marches of temperature for these two heights above the soil, in localitics where the soils are similar in physical character, color and moisture-content, as is the case for the two stations here considered. The soils surrounding the plant cultures at both Oakland and Laston are rather heavy loams with brown 
top soil. They are somewhat strongly retentive of moisture, and are thus apt to be rather cold as compared to the air above them. It thus appears that the temperature conditions to which the culture plants were exposed at Easton and at Oakland may be safely compared, for the present purpose, by means of the thermometer readings obtained from the instruments in the elevated shelter's.

Plant exposure. The plant cultures were protected from accidental injury by enclosing the cultures at each station in a wire-covered frame. The

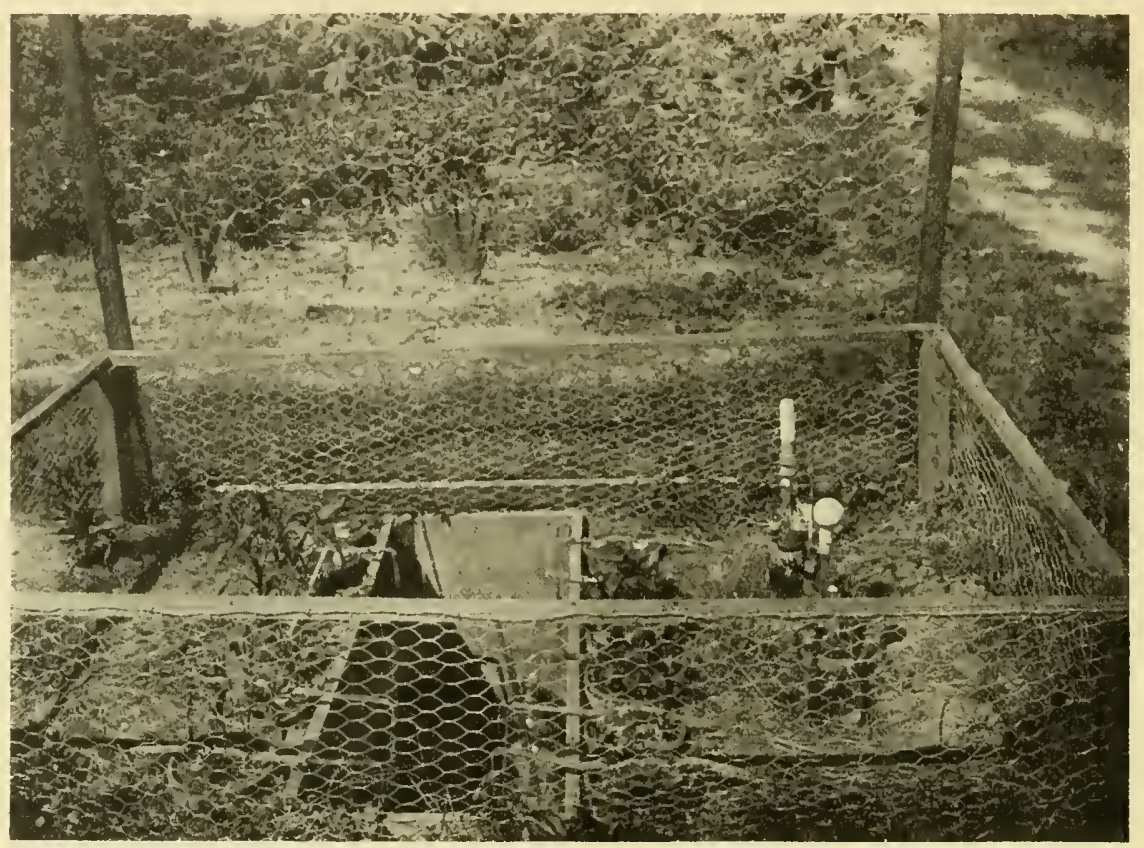

Fig. 4. Interior view of culture enelosure, with top raised, showing irrigator pit, plunged pots and atmometer. (The spherieal atmometers were being subjected to preliminary tests and their readings are not eonsidered in the diseussion.)

form and arrangement of this is shown in figure 4. It was rectangular, 1.22 m. ( $4 \mathrm{ft}$.) from north to south, $1.83 \mathrm{~m}$. (6 ft.) from east to west, $45 \mathrm{~cm}$. (18 in.) high, and was surrounded with galvanized iron wire netting having meshes $2.5 \mathrm{~cm}$. ( $1 \mathrm{in}$.) in diameter. This cage was provided with a removable top consisting of a frame covered with wire netting, with meshes $5 \mathrm{~cm}$. (2 in.) in diameter. A pit $61 \mathrm{~cm}$. (2 ft.) deep, $1.22 \mathrm{~m}$. (4 ft.) long from north to south and $41 \mathrm{~cm}$. (16 in.) wide, was dug across the center of the enclosure, and was walled up with boards. The plant cultures, in ordinary flower pots, were arranged in two rows of six each just outside of the pit, one row 
along each of its longer sides. The pots were plunged to such a depth that the soil surface of each pot was about level with that of the surrounding soil.

As already stated, a new culture for each of the four plant species was started at each station approximately once every two weeks, and each culture was continued for four weeks. Thus the culture periods overlapped, and there were regularly two sets, of four pots each, at each station. In addition to these, a third set of four pots, without plants, was constantly maintained at each station. The twelve pots, which were thus always present after the third visit to each station, were arranged in two rows running from north to south, a row of six on either side of the pit, and were so placed as to avoid as much as possible having the plants of the different cultures shade each other. The successive sets of four cultures each, were placed, two pots on each side of the pit, beginning with the north end, so that the younger plants were never directly north of the older ones. Also the cultures of the different species of the same age were arranged so that the more vigorous growers would not shade the slower-growing forms. Thus, Windsor bean was placed north of the corresponding soy-bean culture on the east side of the pit, and maize was placed north of the corresponding wheat culture on the west side.

The soil conditions. The same character of soil was used in all of the plant cultures. It was a rather light soil obtained from an untilled field near College Park, Md., and was of the soil type classified as Norfolk sand by Bonsteel. ${ }^{8}$ Its water-retaining power was found to be 43 per cent., on the basis of dry weight, by the Hilgard ${ }^{9}$ method, which employs a onecentimeter soil column. The top-soil was removed from a small area to a depth of $15 \mathrm{~cm}$. (6 in.), and the soil thus obtained was thoroughly mixed and sifted. It was then placed in cloth sacks and shipped to the various stations, where it was stored in air-dry condition, in covered, water-tight, galvanized iron cylinders, until needed for use in the cultures.

The soil containers for the cultures were ordinary porous clay flower pots, in form like the frustrum of a cone, being smaller at the bottom. Their inside dimensions were: top diameter, $15 \mathrm{~cm}$.; bottom diameter, $9.5 \mathrm{~cm}$.; height, $16 \mathrm{~cm}$. The capacity of each pot was thus approximately 1980 ec. of soil, when level full. However, in filling the pots, two auto-irrigator cups (to be considered below), each occupying about $72 \mathrm{cc}$., of volume, were also placed in each pot, and the soil was compacted so that it stood about $1 \mathrm{~cm}$. below the top of the pot. The actual volume of soil in the pots when in use was approximately $1645 \mathrm{cc}$.

In order to secure uniform soil conditions in the cultures, it was necessary not only to have soil of similar character for all cultures, but also to bring

\footnotetext{
${ }^{8}$ Bonsteel, Jay A., The soils of Prince George's County. Publication of the Maryland Geological Survey. Baltimore, 1911.

9 Hilgard, E. W., Soils, their formation, properties and composition. New York, 1911. Page 209.
} 
it into the same physical condition, so that it would retain its structure during the growth period of the plants and so that all cultures of the same age should have practically the same soil conditions. The very desirable condition of loose tilth could not be maintained in these cultures; since they were freely exposed to the weather and were visited only once in a fortnight, every rain must pack loose soil more or less, and heavy rains would saturate it completely. Therefore, to put the soil into a state of aggregation to be least altered by varying weather conditions, the soil was saturated "with water immediately after it was put into the pots. This was accomplished by plunging the pots of soil into a bucket of water, and allowing them to remain submerged until air bubbles ceased to rise. The pots were then set in position in the enclosures and allowed to drain. After the soil had settled, its surface was found to be one centimeter below the top of the pot, the soil mass having been compacted from 1736 to 1646 ce., or approximately 5.5 per cent. of its loose volume.

The soil moisture in the cultures was maintained by means of Livingston auto-irrigators. ${ }^{10}$ This device, as here used, consisted of two eylindrical porous clay cups (of the regular form supplied by the Plant World) $15 \mathrm{~cm}$. long and $2.5 \mathrm{~cm}$. in diameter. These were connected with each other and with the water reservoir by glass tubes in the form of an inverted $J$. The cups were placed vertically in the pot, their rubber-stoppered tops level with the soil surface, and were so arranged as to supply water to the soil against a pressure of $35 \mathrm{~cm}$., or more, of water column. By this arrangement water is withdrawn from the porous cups by the capillary attraction of the water films in the soil about them. The difference between the pressure of the water in the cups and that of the soil films was adjusted in these experiments by placing the 1-gallon (nearly 4-liter) water reservoirs in the pits above described, $60 \mathrm{~cm}$. below the surface of the soil, so that the water level in the reservoir, when the latter was full, was $35 \mathrm{~cm}$. (14 in.? below the soil surface. The moisture content of the soil in the pots mas thus maintained so that it was never less than about 10 to 13 per cent. (on the basis of dry weight), in which condition this particular soil was rather too wet than too dry for the best growth of the plants here studied. The soil was often moistened by rains, which sometimes inereased the moisture content up to its maximum water-retaining power for the $15-\mathrm{cm}$. soil columns in the pots (approximately 23 per cent.).

To prevent the movement of water and dissolved salts through the sides of the pot, between the culture soil and that surrounding the pot, the pots were painted on the outside. This coating proved inefficient, however, as the paint soon peeled off. The pots were Iater surrounded with strips

10 Livingston, B. E., A method for controlling plant moisture. Plant World 11: 39-40. 1908.

Hawkins, Lon A., The porous clay cup for automatic watering of plants. Plant World 13: 220-227. 1910.

Transeau, E. N., Apparatus for the study of comparative transpiration. Bot. Gaz. 52: 54-60. 1911. 
of oilcloth, which appeared to be more satisfactory. Whatever water movement occurred through the pots, however; was probably in the direction from the culture to the outside soil, as the soil in the pots was at all times more nearly saturated than was the soil around them. Thus there is little probability that the soil solution in the pots became greatly modified by the entrance of soil water from without.

After preparing the pots and arranging the watering devices as described above, the pots were then allowed to remain fallow for about two weeks before planting. Thus the soil was fully drained out after the preliminary saturation, and had settled into a condition somewhat nearly approaching that of structure-equilibrium, before the seeds were planted. Care was taken to space the seeds uniformly, and to place them about equally distant from the auto-irrigator cups and from the sides of the pots, so that all should have, as nearly as possible under the conditions of the experiments, the same soil moisture conditions.

At the time of planting, and at each fortnightly visit thereafter as long as each culture was continued, a soil sample was taken from each pot for the purpose of soil moisture determination. The method used was that described by Brown. ${ }^{11}$ A small cylinder of soil containing about 22.5 cc. (about 30 grams, dry weight) was removed by means of a brass tube (cork borer), which was thrust into the soil vertically at a point midway between the two auto-irrigator cups, and about $3.75 \mathrm{~cm}$. (1.5 in.) from each cup. Each soil sample thus taken represented a vertical section of the soil mass, of uniform diameter and extending from the upper surface to the bottom of the pot. The soil samples thus obtained were immediately transferred to heavily paraffined paper-pulp containers (which were serially numbered), and sealed in these with a paraffin seal. They were then sent, in pasteboard mailing-tubes, to Baltimore, where the moisture content was determined. Previous to the beginning of this experiment, the type of paraffined container here used (which is on the market for milk, etc.) was tested, as to its permeability to water vapor and it was found that the greatest loss from moist soil left sealed in such vessels for an entire week was not greater than about 0.1 per cent. of its dry weight. None of the field samples were ever left in the containers for a longer period than this, so that errors in the soil moisture determinations due to leakage may be regarded as negligible. For lightness in transportation and for general ease in handling, these paper containers were found very satisfactory. Larger ones of the same kind were employed for storing the stock of seeds.

When the plants were removed from a pot (about six weeks after that pot was filled) the soil was discarded, and fresh soil from the stored supply. Was always used in refilling.

11 Brown, W. H., The relation of evaporatis: ts the water content of the soil at the time of wilting. Plant World 15: 121-134. 1912. 


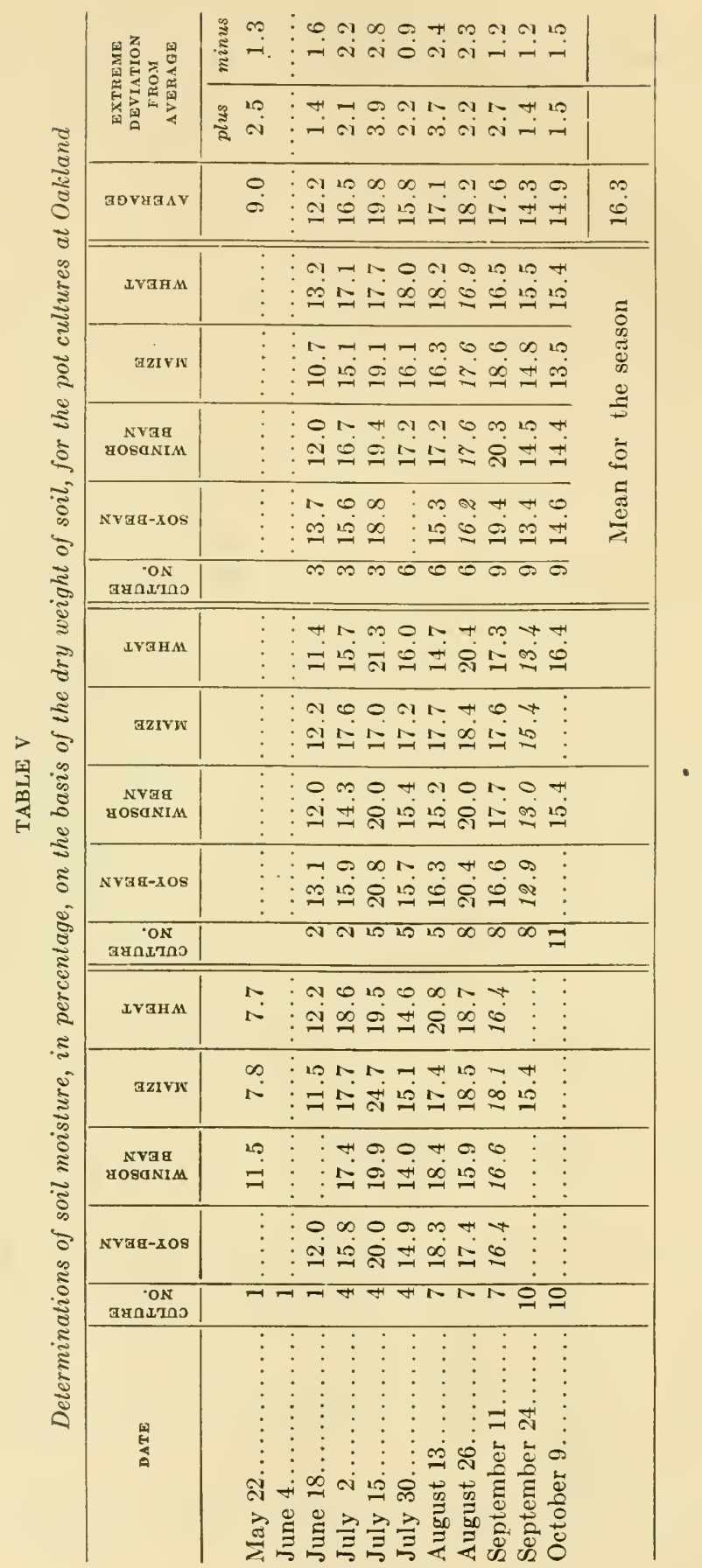


Climatic Conditions in Maryland

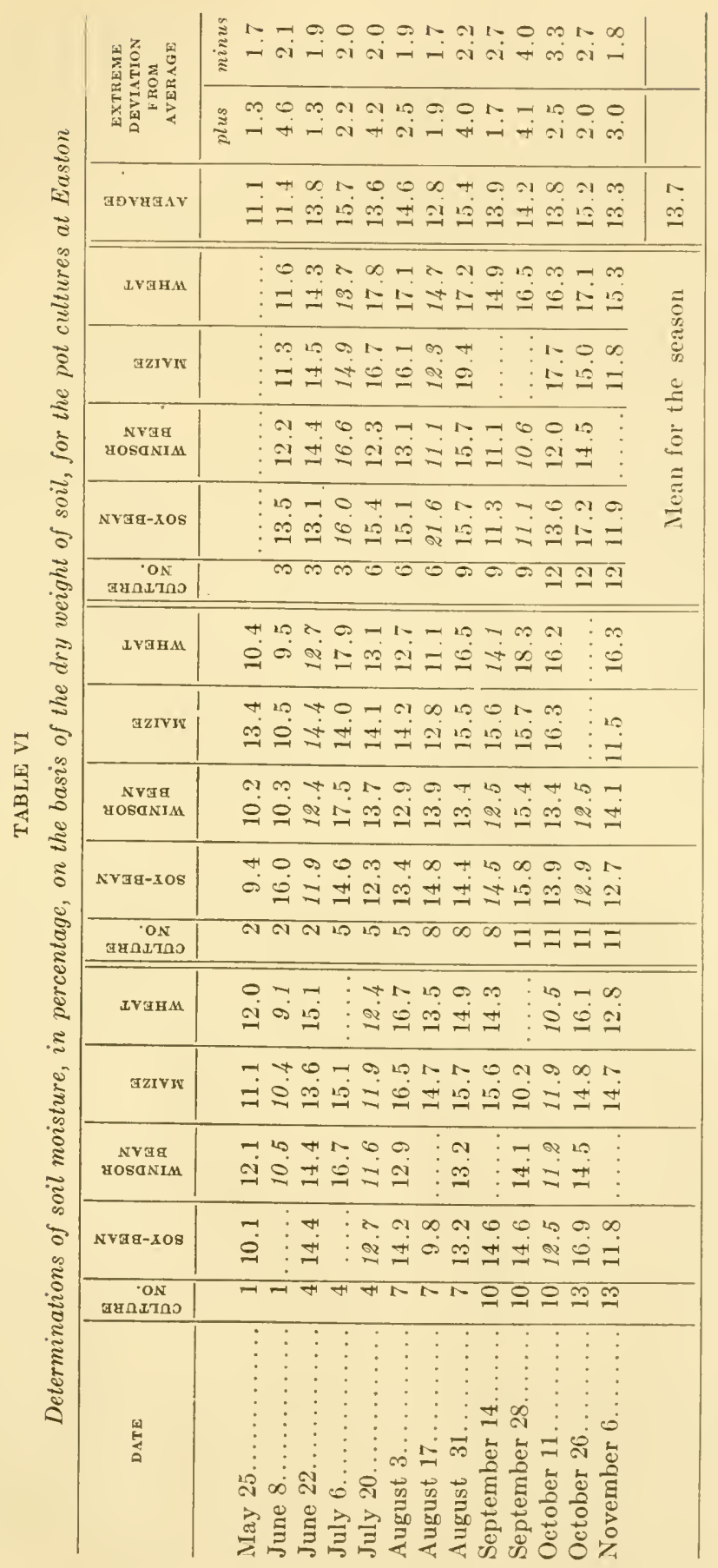


The results of the soil moisture determinations at Oakland and at Easton are shown in tables $\mathrm{V}$ and VI. These are given in detail, for all four species of each culture, to show the variations. The average of all determinations for each day of observation, together with the extreme deviations from the average, are given in the last column. In the cases where no data are given the auto-irrigating device was accidentally broken, and consequently the soil dried out.

In these tables, each date given in the first column applies throughout the line in which it stands. Thus, on July 15, the second sample was obtained from culture 4 , the first from culture 5 , and the third from culture 3. The kind of plant grown, or to be grown, in the pots is shown above, at the head of each column.

The soil moisture contents of samples taken from different pots at the same station and on the same date are fairly consistent, as is shown by the column of extreme deviations, which generally show values between 1 and 4 , approximately, or from about 7 to 25 per cent. of the average soil moisture percentage. The values of the average soil moisture contents are quite variable, however; these averages range in value from 9.0 to 19.8 for Oakland (table V), and from 11.1 to 15.7 for Easton (table VI). These variations seem probably to have been due to differences in the climatic features that affect soil moisture, namely rainfall and evaporation. The irrigators simply kept the moisture content of the soil from becoming lower than about 10 per cent.

\section{GENERAL MOISTURE CONDITIONS AND THEIR MEASUREMENT}

The water-supplying and water-withdrawing influences exerted by the atmospheric surroundings of ordinary terrestrial plants are to be grouped under two headings; precipitation and evaporation. The first of these (precipitation or rainfall) exerts its main influence upon ordinary plants indirectly, by increasing soil moisture and, consequently, the facility with which the soil may supply water to the plant roots. Precipitation was measured in the usual manner; the amount of moisture intercepted by the funnel of a standard U. S. Weather Bureau rain gage was measured each evening at sunset, and the result was computed as depth of rainfall in inches.

In the present study interest in the environmental moisture conditions centers mainly about the influence exerted by the surroundings to alter the possible rate of water supply or the actual rate of water loss from plants. For ordinary terrestrial plants, which absorb practically all of their water from the soil in which they are rooted, the water supplying capacity of the surroundings is mainly determined by the resistance offered by the soil to water-absorption by plant roots. ${ }^{12}$ This resistance varies with the character and

12 For a more thorough discussion of this general topic, and for accounts of applications of this principle, see: Livingston, B. E., and Hawkins, Lon A., The water-relation between plant and soil. Carnegie Inst. Wash. Pub. 204: 3-48. 1915. Pulling, H. F., and Livingston, B. E., The water-supplying power of the soil as indicated by osmometers. Ibid. 204: 49-84. 1915. 
structure of the soil and with its moisture content. Since soil of the same character and-structure was employed in all of these experiments, the soil moisture content of these cultures may be considered as an approximate index of the power of the soil to supply water to plant roots.

The envirommental conditions most influential in determining the rate of water-loss from plants are the evaporating power of the air and the intensity or heat-equivalent of the absorbed radiant-energy, received from the sun. ${ }^{13}$ The first of these was measured by atmometers, and the measurements will here be considered as indicating the degree of the tendency of the environment to promote evaporation from the plants. Radiant energy was not measured in this study, though a consideration of the available sunshine data will be presented farther on.

The influence of the evaporating power of the air upon plants is twofold. It may affect the water supply of the plants indirectly, by reducing the soit moisture, and it withdraws water from them directly, by evaporation (transpiration): The evaporating power of the air was measured in these studies by means of cylindrical porous cup atmoneter's ${ }^{14}$ located inside the culture enclosures, $45 \mathrm{~cm}$. (18 in.) from the east end and $30 \mathrm{~cm}$. (1 ft.) from the north side (fig. 4). The atmometer cups were provided with rain-correcting mereury valves, and were mounted upon reservoir bottles of $500 \mathrm{cc}$. capacity. The reservoirs were placed upright in the soil, and at such a depth that the centers of the atmometer cups were from 28 to $32 \mathrm{~cm}$. above the soil surface. These atmometers were refilled with distilled water and their water-loss was recorded at every fortnightly visit to each station. After every reading each atmometer was replaced by another that had just been standardized, and the used cup was returned to Baltimore, where it was restandardized. All atmometer readings were reduced to terms of the Livingston cylindrical standard, and they should thus be directly comparable with other measurements on the same basis. The spherical cups shown in figure 4 were employed only for preliminary tests of various makes of these instruments. They were not yet perfected at the time this work was carried out.

The effects of rainfall and evaporation upon soil moisture in the cultures here considered were much reduced by the use of the auto-irrigators, which gave, as has been stated, a minimum soil-moisture content of approximately 10 to 13 per cent., on the basis of dry weight. The soil always appeared dark-colored and moist on the surface, and felt damp to the touch. Rain

13 Livingston, B. E., Light intensity and transpiration. Bot. Gaz. 52: $417-435.1911$.

14 Livingston, B. E., A rotating table for standardizing porous eup atmometers. Plant World 15: 15\% -162 1912. Other references are there given.

- Atmometry and the porous cup atmometer. Plant World 18: 21-30, 51-i4, 95-111, 143-149. 1915.

Shive, J. W., An improved non-absorbing porous eup atmometer. Plant World 16: $7-10.1915$.

Johnston. E. S., and B. E. Livingston, Measurement of evaporation rates for short time intervals. Plant World 19: 136-140. 1916. 
thus influenced the soil moisture content of the cultures only by increasing an already abundant supply of water. The general character of the effects of variations in precipitation and in the evaporating power of the air upon the soil moisture content in these partially controlled plant cultures may be seen from comparisons of the seasonal marches of these three factors, which are shown as graphs, for Oakland and for Easton, in figures 5 and 6, respec-

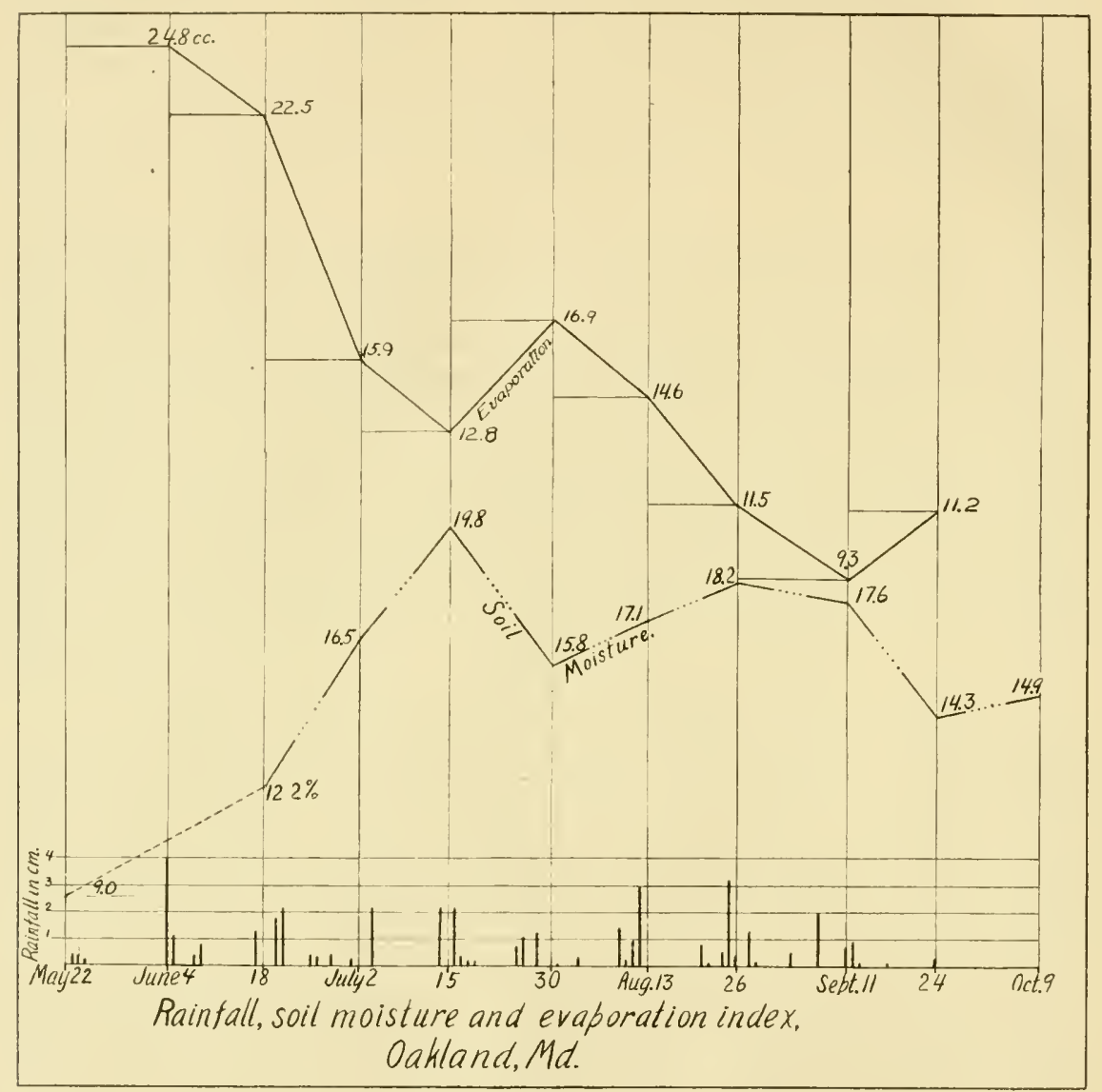

Fig. 5

tively. The abscissas here represent time and season, and the ordinates (the values of the various data) are shown at the dates on which measurements were taken. The daily amounts of rainfall are represented at the bottom of each figure, as vertical lines, the relative lengths of which indicate the depths of rainfall. Soil moisture is given as percentage on the dry weight of the soil as basis. Each ordinate represents the average of all 
samples taken on the sane day, these values being obtained from tables $\mathrm{V}$ and VI. Soil moisture data for June 4 at Oakland (fig. 5) are lacking, since the pots were saturated by rain at the time of observation, and the graph is drawn as a straight, broken line between the point for May 22 and that for June 18. Evaporation is expressed as the average rate of loss from the standard cylindrical porous cup, in cubic centimeters per day, for each culture period of approximately two weeks. The duration of the period to which each rate applies is indicated by the length of the horizontal line drawn to the left of the corresponding point on the graph of

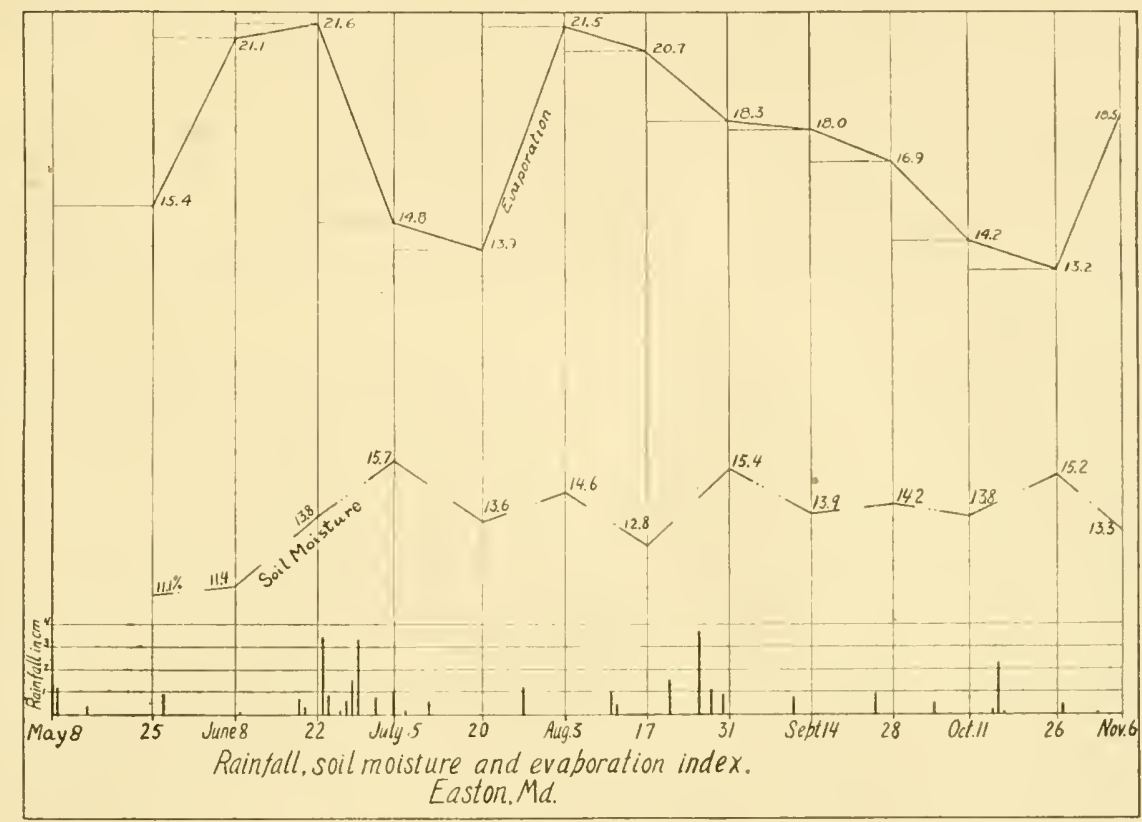

FIG. 6

evaporation. Evaporation was not measured for the last two-week period at Oakland (Oct. 9, Fig. 5), since the atmometer cups had been broken by freezing.

These graphs show an evident relation between rainfall and soil moisture, which is especially apparent in figure 5, for Oakland. Here the highest observer moisture content occurred on July 15 , the day after a rain of 2.16 cm. (0.85 inch), and the other high values, for August 26 and September 11, were likewise due to rains, occurring in each case on the same day as that on which the soil sample was taken. These instances indicate that the effects of rain might persist in the culture pots for at least as long as two days. This is not always necessarily the case, however, as is shown by the 
observation for June 18. A rain of $1.27 \mathrm{~cm}$. (0.5 in.) occurred on June 17, but the soil moisture content on the next day was somewhat below the average, being only 12.2 per cent. This rapid decrease in soil moisture was probably due to a high rate of loss by evaporation during the period between the cessation of the rain and the time of the observation. The general effect of high evaporation rates in reducing the soil moisture content of the culture pots is suggested in the seasonal marches shown by these graphs for Oakland. In every instance, excepting one (Aug. 26-Sept. 11), the graph for soil moisture slopes in the direction opposite to the direction of slope shown by the graph for evaporation for the corresponding time period. A similar relation is distinguishable in the graphs of figure 6, for Easton, though the relation between the two is here not so clear as in the other case. Soil moisture determinations were not made frequently enough, however, to show the detailed seasonal march of this condition in the cultures, and an exact study cannot be carried out in this connection. The evidence presented indicates clearly that the soil moisture content was greatly influenced by both rainfall and evaporation, in spite of the employment of the irrigators.

The probable effect of a given amount of rain upon the moisture content of the soil in one of these culture pots may be approximately computed from the amount of water required to increase the moisture content from the value of the average maintained by the auto-irrigators to a value representing the maximum water-retaining power of the soil-mass in the pots. The difference between these two latter values may be taken as the amount of water that, falling upon the exposed soil surface of the pot, would cause the maximum increase in soil moisture content. Run-off if not important in this calculation, as the side walls of the pots, extending a centimeter above the soil surface, effectively prevented its occurrence, excepting for the very heaviest rains, and drainage of water through the bottom of the pot should not be very pronounced until the soil mass had become moistened to above its maximum water-retaining power. The average dry weight per cubic centimeter of the soil, as calculated from fifty-two samples each containing about 22.4 cc., proved to be $1.4 \mathrm{~g}$. The $1650 \mathrm{cc}$. of soil in each pot thus weighed about $2300 \mathrm{~g}$., in the dry state. The maximum water-retaining power of the soil here used, as shown by the samples taken soon after rains, was nearly 23 per cent., on the basis of the dry weight of the soil, while the average moisture content maintained in the pot between rains, by the irrigators, was about 14 per cent. Therefore, $207 \mathrm{cc}$. of water, added to the soil in its normal condition in the culture, would be all that could be retained against the downward attraction of gravity, for this amount corresponds to an increase in soil moisture content of 9 per cent., on the basis of dry weight. The area of the top of a pot of the type here used is approximately 
$200 \mathrm{sq} . \mathrm{cm}$., so that a depth of rainfall of $1.03 \mathrm{~cm} \cdot{ }^{15}(0.415$ inches $)$ received by a culture, after a period of drought, would be sufficient to bring the soil to its maximum water content, 23 per cent. On the basis of this calculation, the cultures at Oakland must have been saturated by at least ten storms during their combined growth period of twenty weeks, and those at Easton by seven or eight storms during their combined period of twenty-six weeks. Every light rain must also have exerted some influence upon the soil moisture. Under such varying conditions, the fortnightly soil moisture deteminations become of little significance, except to show the general magnitude of the variations occurring during the season, and to indicate the relations holding between these and their external causes.

As has been emphasized by Livingston and Hawkins, the environmental moisture conditions influencing plant growth, neglecting the influence of sunshine, may be considered as represented by the relation between the power of the soil to supply water to the plant roots and the power of the aerial environment to remove water by transpiration. Not being able as yet to measure the former of these two terms, but with due regard to the prime importance of the soil moisture content in determining the power of the soil to deliver water to plant roots (especially in such cultures as those here dealt with, where the soil was all alike excepting for its moisture content), an approximation of this value may be obtained by substituting the main component factor for the whole term of water-supplying power. This approximation may then be stated: the entire moisture relation of these plants is approximately represented by the relation between soil moisture content and the evaporating power of the air. This relation (expressed as a ratio) has been employed with considerable success by Shreve, ${ }^{16}$ in studies of the relation between climatic and soil conditions, on the one hand, and plant distribution on the other. But such a ratio cannot be used in the present studies, since, as has been remarked, the soil moisture contents of the pots were not deternined frequently enough to supply the needed data. From the information above set forth, and on general a priori grounds, a still less precise approximation may be attained by substituting in the place of the soil moisture content the main factor tending to increase this content, namely rainfall. Thus modified, the above statement becomes: the moisture relation of these plants may be approximately expressed as the ratio of rainfall to the evaporating power of the air. This ratio, as will be seen at once, involves nothing but climatic conditions, and both of these two conditions were measured in the present study. Comparisons between such ratios for different time periods and for different localities should show, in a general way, the relative tendencies of the different climatic complexes to maintain

15 This estimate is too high, since the retaining power of the soil was determined witl a l-cm. soil column and the column in the pot was much higher than this.-B. E. L., Ed.

16 Shreve, F., lainfall as a determinant of soil moisture. Plant World 17: 9-26. 1914.

PHYSIOLOGICAL RESEARCHES, VOL. 2, No. 4 ,

SERIAL NO. 14, FEBRUARY, 1917 
water in any water-absorbing substance (such as soil or a plant). The ratio here proposed is the reciprocal of the ratio of evaporation to rainfall, as used by Transeau ${ }^{17}$ which expresses the drying tendency of the climatic environment. It is of course not important which form of ratio is employed, since one is the reciprocal of the other, but the writer finds it easier to think of an influence tending to moisten or to maintain moisture in an object than to think of an influence tending to withdraw water.

In using rainfall as a measure of the general supply of moisture for plants, it is assumed that all of the water falling is effective to increase the moisture of the soil, which is the direct source from which plants absorb water. This assumption is not ordinarily strictly true, however, as has been clearly pointed out by Shreve, and as has been demonstrated also for the pot cultures here employed. As shown above, any rain in excess of $1.03 \mathrm{~cm}$., occurring in a single shower, should have been without effect upon the soil moisture content in these cultures, this moisture content being already at its maximum. Thus, the rainfall-evaporation ratios as here derived are probably generally too high.

\section{TEMPERATURE CONDITIONS AND THEIR MEASUREMENT}

Maximum and minimum temperature readings. The temperature data used in this study were all obtained from maximum and minimum thermometers read daily at sunset. The results of these readings are shown graphically in figure 7 , in which the abscissas represent the successive dates of the daily observations, and the ordinates are the recorded maximum and minimum temperatures, in degrees Fahrenheit. The upper two graphs of figure 7 show the maximum and minimum temperatures recorded at Oakland during the period of observation, and the lower two present the corresponding data for Easton.

The temperature conditions at the two stations, as shown in figure 7, exhibited large and irregular fluctuations, this being especially true of the western station. Both the daily range of temperature (within each 24 hour period) and the interdiurnal fluctuations (variations of the average conditions for different 24 hour periods) were greater at Oakland than at Easton.

\section{TEMPERATURE WEIGHTINGS AND INTEGRATIONS}

General discussion. It is obvious that this complex mass of temperature data must be greatly simplified before it may be compared to the plant growth measurements, which were obtained only at relatively long intervals. Two general lines of procedure have been proposed for simplifying such complex series of temperature observations, (1) the grouping of the

17 Transeau, E. N., Forest centers of eastern North America. Amer. Nat. 39: 875-889. 1905. 
temperature data into several classes and the computation of the lengths of the time periods during which each class obtains, and (2) the summation of the temperature data for certain seasonal periods. The method of temperature classes was developed by Koeppen ${ }^{18}$ and has been modified in various ways by later writers. ${ }^{19}$

In making such temperature summations as those just mentioned either the data for any given time period may be employed directly or they may be replaced, before the summation is performed, by measures of the temperature efficiency, obtained by weighting each themperature magnitude in accordance with its observed or probable effectiveness in promoting plant

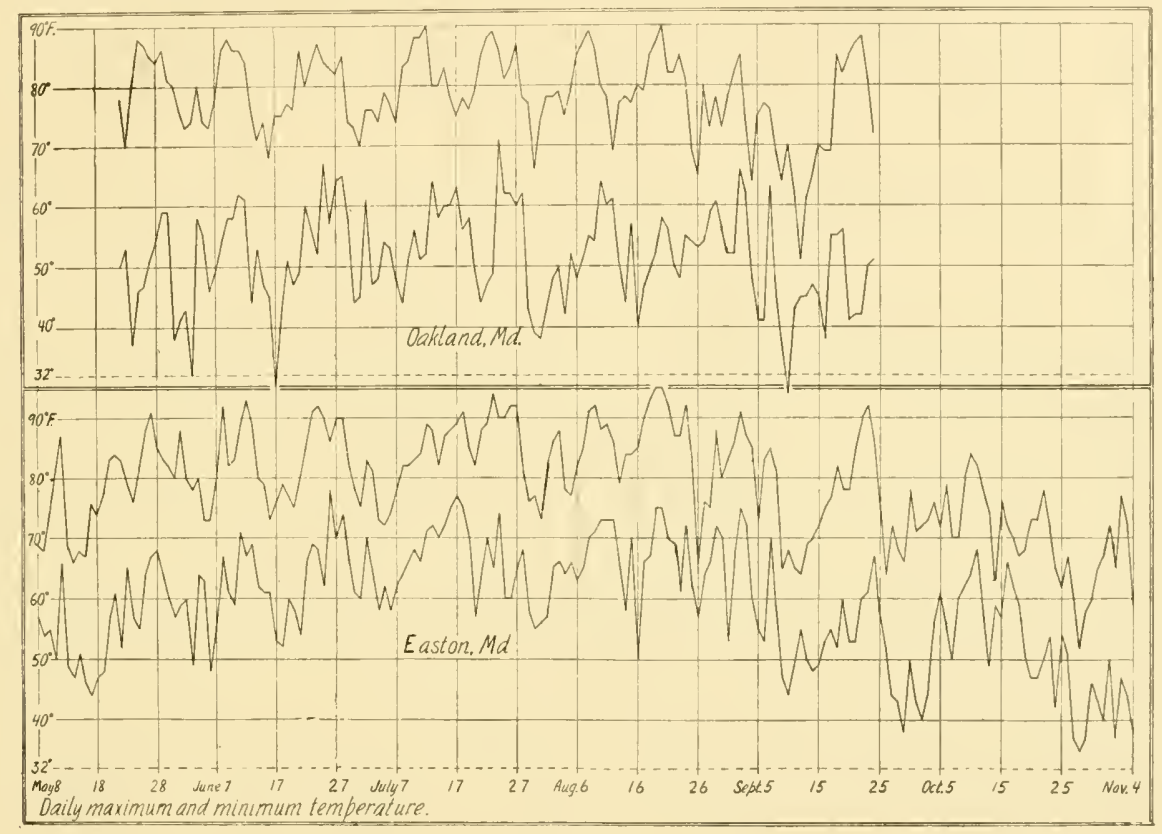

FIG. 7

growth. Until more real information is at hand regarding the effects of different degrees of temperature upon growth processes, such methods are mainly of value in defining and comparing climates as such, for which purpose they have been employed by both of the writers mentioned above.

Another somewhat similar method of simplying temperature data is that proposed by MacDougal. ${ }^{20}$ The average hourly rates of growth are

\footnotetext{
18 Koeppen, W., Die Wärmezonen der Erde, nach der Daueı der heissen, gemässiğten und kalten Zeit und nach der Wïkung der Wärme auf die organische Welt betrachtet. Meteorol. Zeitchr. 1: 215-226. 1584.

19 See especially: Zon, R., Meteorological observations in connection with botanical geography, agriculture and forestry. Monthly Weather Rev. 42: 217-223. 1914.

$20 \mathrm{MacDougal}, \mathrm{D}$. T., The auxo-thermal integration of climatic complexes. Amer. Jour. I3ot. 1: 186-193.
} 1914. 
to be computed for some given plant form, for time periods during which the temperature ranges over specifie intervals on the thermometer scale (intervals such as 40 to $45^{\circ}, 45$ to $50^{\circ} \mathrm{F}$., etc.). Then the number of hours representing the duration of natural temperatures lying within each of the given ranges, for any given longer period (day, week, month, growing season, etc.) is ascertained from thermograph records, and the product of the average hourly growth rate for each range by its number of hours is considered as the total temperature efficiency for that range of temperature conditions. Such efficiencies are computed for all of the temperature ranges experienced during the time period considererl, and the sum of all of these efficiencies represents the total temperature efficiency of the larger period, for promoting plant growth. The early literature referring to temperature integrations is reviewed by Abbe, ${ }^{21}$ and need not he considered further here.

Laboratory studies carried out with plants have shown that plant growth rates are more or less directly related to temperature, the best study bearing on this matter being the recent one of Lehenbauer. ${ }^{22}$ If plants are subjected to temperatures of different magnitudes maintained for definite time periods, all other conditions being kept approximately uniform, the growth rates are found to vary in a characteristic manner with the temperature conditions. Below a certain low temperature and above a certain high temperature no growth takes place, these limits being termed the minimum and maximum temperatures for growth. If the growth rates for any plant form, when subjected to different temperatures, are expressed in the form of a graph, the temperature values being the abscissas and the growth rates of the plants being the ordinates, then the graph takes the form of a curve which shows a slow increase in rate of growth for temperatures just above the minimum, followed by a rapid increase until the highest growth rate is approached. Then the increase in growth rate with rise in temperature becomes slow again until the highest point of the graph is reached (the latter point being termed the optimum), when the growth rate rapidly descends to zero with still further increase in temperature. Whether each of the temperatures experienced during the constantly varying conditions ordinarily encountered out-of-doors will affect the growth of plants in the same manner as does a similar maintained temperature, as usually employed in laboratory tests, may only be found out by actual trial. It may be tentatively supposed, however, that plants respond to the varying temperatures of nature in a manner at least similar to that in which they react to maintained temperatures, and indices formulated on this basis

${ }^{21}$ Abbe, Cleveland, First report on the relation between climate and crops. U. S. Weather Bureau, Bitll. 36. 1905. Pages 169-343.

22 Lehenbauer, P. A., Growth of maize seedlings in relation to temperature. Physiol. Res. 1: 247-288. 1914. Earlier references to the general subject are there given. 
may be tested by comparing them to actual rates of plant growth, as observed out-of-doors.

The use of direct summations of the daily, etc., temperature values is based on the supposition that, within the limits of the temperature range encountered in any given set of investigations, the rates of the plant process studied are approximately proportional to the indices of temperature efficiency obtained by such summations. In other words, if a graph is constructed to show the relation holding between temperature index values and growth rates, the indices of tempcrature being abscissas and the growth increments for corresponding periods being ordinates, then the graph should take the form of a straight line. Laboratory studies of the elongation of roots and shoots of seedlings indicate that the relation between growth rate and temperature is not really a linear one, but that the linear relation is approached excepting near the optimum and minimum. Thus, for what may be regarded as medium temperatures (intermediate between minimum and optimum) for a given plant form, temperature indices derived from summations may be expected to approximate the actual efficiencies of the corresponding temperatures.

Many workers have sought a general law which would express the relation of physiological processes in general (including plant growth) to temperature conditions. The chemical principle of van't Hoff and Arrhenius (which states that the velocity of many chemical reactions somewhat more than doubles for each rise of $18^{\circ} \mathrm{F}$. in the temperature of the medium) has been found to apply quite well to several physiological processes, such as photosynthesis, respiration, germination of seeds, etc., but, of course, only within certain limits of temperature. The literature bearing upon the application of this principle has been reviewed by Livingston and Livingston, ${ }^{23}$ who propose the use of temperature indices computed on the basis of the van't Hoff-Arrhenius law, employing a temperature coefficient of 2 , and considering the rate of growth at $40^{\circ} \mathrm{F}$. as the unit of temperature efficiency. As pointed out by these writers, such indices may not be expected to hold for all temperatures or for all plants.

Temperature efficiencies based upon the application of the van't HoffArrhenius principle take account of the fact that the relation thus supposed to hold between temperature and plant growth is not at all a linear one. Such efficiencies, expressed graphically, present a logarithmic curve. According to the van't Hoff-Arrhenius principle an increase of one degree in temperature is more effective with relatively high temperatures, in accelerating chemical reactions, than is a similar increase with lower temperatures. As we have seen, laboratory studies of plant growth show that the increase in rate of growth is also acclerated with increase in temperature, up to a

${ }^{23}$ Livingston, B. E., and G. J. Livingston, Temperature coefficionts in plant geography and climatology: Bot. Gaz. 56: 346-375. 1913. 
certain limit. Out-door plants, subjected to varying conditions, may be expected to respond to temperature variations in a manner which accords more nearly with the van't Hoff-Arrhenius principle, than with the principle which supposes that the growth rates are proportional to the temperatures themselves, above a certain point on the thermometer scale.

Direct summations of daily means above $40^{\circ} \mathrm{F}$. Direct summations of temperature readings appear to have considerable value, as a means for roughly comparing the temperature conditions affecting plants growing outof-doors, and have therefore been computed for the various growth periods of the plant cultures used in the present investigation. To reduce the temperature readings on the Fahrenheit scale to effective temperatures for the growth of soy-bean, a daily mean temperature value of $40^{\circ} \mathrm{F}$. was here taken as the minimum or zero for growth. No experimental data appear to be on record in the literature, upon the relation of the growth rate of soy-bean to temperature, but it has been found for many other plants that growth ceases, with falling temperature, at approximately $40^{\circ}$. to $43^{\circ} \mathrm{F}$, and therefore $40^{\circ}$ may be taken as the minimum for the plants here used, without introducing the probability of great error. Having thus established a minimum, the effective temperature values were computed by substracting 40 from each successive daily mean. The remainders thus obtained for the successive days of each period were added, and the sum of the effective day-degrees thus obtained was treated as a measure of the temperature efficiency for the period. Such direct summations were made for all of the culture periods and for both stations here dealt with, and the results are given in tables IXXII, which will be described below.

Temperature summations obtained by use of the chemical coefficient. The chemical temperature efficiencies proposed by Livingston and Livingston may be computed from the data of ordinary fluctuating outdoor temperatures in at least three ways: (1) The efficiency index of the average temperature for the whole period may be multiplied by the number of days in the period and the product thus obtained may be treated as the total efficieney. If the van't Hoff-Arrhenius law is applicable to the growth of plants subjected to the fluctuations of temperature encountered in the investigation here reported, then the temperature efficiency for each culture period, computed in the manner just clescribed, should exhibit at least as close a correlation to the corresponding growth rate as does the corresponding direct summation of effective temperatures. (2) The indices for the successive daily mean temperatures may be summed for each period, thus giving due weight to the interdiurnal variations in temperature. (3) The indices for the maximum and minimum temperatures may be averaged for each day and the averages thus obtained may be summed throughout the period. In this way account is taken, not only of the interdiurnal variations but also of the daily range of temperature. Each of these three methods of computa- 
tion has been employed for each of the culture periods and for each of the stations here considered, the results being summarized in tables IX-XII, which will be considered below.

\section{LIGHT CONDITIONS AND THEIR APPROXIMATION}

The present state of our knowledge regarding the relations to plant growth, of the different wave-lengths of radiant energy that we term light, is still less satisfactory than is our knowledge regarding the temperature relation of plants. The portions of the solar spectrum that are most effective in promoting certain plant processes are recognized in a general way, but the quantitative aspects of the problem here brought up have been but little investigated. Most of the studies that have been carried out in this connection have clealt with sunlight, and have treated the solar radiation as if it were constant in composition, if not in intensity, but of course it is not constant in either respect. Furthermore, while it is well known that only the light of certain portions of the solar spectrum is important for plant growth, the only data available for the evaluation of the sunlight for any locality for any period of time are rather rough, general estimates of the duration and of the heating. effect of the total radiation received.

The duration of sunshine in hours per day (that is, the duration of light with a heating effect above a certain very roughly defined minimum) is measured by the U. S. Weather Bureau only at its regular stations. The sunshine records for the coöperative stations, such as Oakland and Easton, are only the local observer's ocular estimates of clear, partly cloudy and cloudy days. This latter form of record does not appear to be sufficiently precise, nor is it sufficiently detailed, to furnish data for a study of the relations between plant growth and light conditions. Fortunately, however, complete instrumental records of sunshine duration are available for other stations in the vicinity of those here used. Thus the data for Elkins, West Virginia, have been here employed as if for Oakland, and the data for Washington, D. C., and Baltiniore, Maryland, have been averaged, the average being used as if for Easton. The general character of the weather at Oakland is very similar to that at Elkins, the two stations being situated at similar altitudes and about $48 \mathrm{~km}$. (30 $\mathrm{mi}$.) apart. Likewise, the weather conditions at Easton are generally not markedly unlike those at Baltimore and Washington, which are only about 50 and $75 \mathrm{~km}$. (30 and $45 \mathrm{mi}$.) distant. The averages obtained by combining the records for Baltimore and for Washington were comparéd to the local observer's records at Eastun day by day, and the two sets of data showed very few disagreenents as to the character of the days, whether clear or cloudy.

All the sunshine data are presented in tables VII (Oakland) and VIII (Easton), to which reference will be made in the following paragraphs. 
For every culture period there are shown in these tables two approximations of the amount of sunshine available to the plants: (1) the average daily duration of actual sinsnine as derived from the average daily duration of possible sunshine for that period and latitude, and from the local observer's records, and (2) the average daily duration of actual sunshine recorded by the sunshine-recorder at Elkins (for Oakland), and the mean of the mumbers thus recorded at Baltimore and Washington (for Easton).

In the case of the local records of the coopperative observers, days recorded - as clear were treated as whole days of sunshine (column 4), partly cloudy days were treated as half-days of sunshine (column 5), and cloudy days were treated as without any sunshine (column 6). These days and halfdays were summed for each culture period for each station, and the sum was expressed (column 8) as percentage of the total number of days in the sulture period (column 3). Next, the average daily duration of possible sunshine for this region, ${ }^{24}$ for each period (column 7 ), was multiplied by the corresponding observational percentage (column 8), to give an approximation of the average daily duration of actual sunshine at the two stations considered (table VII, column 10; table VIII, column 12).

For each day of the Oakland season the percentage of possible sunshine duration was obtained from the records of the instrument at Elkins. These daily percentages were then averaged for each Oakland culture period, the resulting average daily percentages being given in table.VII, column 9 . Each average daily possible duration of sunshine (table VII, column 7) was next multiplied by the corresponding average daily percentage (table I'II, column 9), to give an approximation of the average daily actual duration of sunshine (table VII, column 11), for each culture period at Oakland.

The percentages of possible sunshine duration for each Easton period were obtained from the instrumental records for Washington and for Baltimore (table VIII, columns 9 and 10), and these two data were then averaged for each period (table VIII, column 11). These composite percentages were each multiplied by the corresponding average daily possible number of hours of sunshine (table VIII, column 7), to give a number that is taken to represent the average daily actual duration of sunshine (table VIII, column 13) for each culture period at Easton. Since the cultures at this station were shaded from possible sunshine for approximately four hours each day, as has been noted, the data of column 13 require to be corrected so as to take this into account. This was done by subtracting from each number in column 13 four times the corresponding percentage in column 11 , since only this percentage of the four hours when the plants might have been in shade is to be considered as sunshine period. The numbers thus corrected are given in column 14 .

24 Derived from data in: Sunshine tables, U. S. Dept. Agric. Weather Bureau. 
It appears that the numbers representing average daily actual sunshine as obtained by the first method (from local observers' records) are generally somewhat larger than those obtained by the second method (from instrumental records for nearby stations). Which of these two series may be more nearly correct eannot be determined, however; both methods are very erude. The data derived by the second method (table VII, column 11; table VIII, column 14) will be employed where sunshine duration enters into the following discussions, but it is possible that the first method may prove of some value in climatic studies related to plant growth, in cases where oeular observations of cloudiness constitute the only available sunshine data.

The sunshine records of the regular U.S. Weather Bureau stations, such as Elkins, Baltimore and Washington, are obtained by means of Marvin sunshine recorders, each of which consists of a pair of themometers, so arranged as to record the lengths of the periods during which the heating power of the sunlight reeeived is suffieiently great to maintain or surpass a certain difference in temperature between a blackened and a transparent glass bulb. The instrument thus records sunshine when the sun is shining brightly, but does not take aecount of differences in the absolute intensities of solar radiation received, except to show that the heating power is above the threshold value for the instrument. On account of the form of its inelined eylindrical bulbs, this threshold ralue does not oceur with the same light intensity for different hours of the day nor for different seasons of the year. This kind of sumshine record is, however, the best that is obtainable at the present time, and it may be supposed to have some possible value as indicator of favorable or unfavorable conditions for plant growth.

The studies of Richter ${ }^{25}$ inclicate that photosynthesis in plants, and consequently the rate of formation of earbohydrates (which generally constitute the greater part of the dry substance of these organisms) is proportional to the amount of light energy actually absorbed by the leaves. From this it may be supposed that the sunshine data here considered may indicate, in a general way at least, the relative amounts of radiant energy arailable for plants during the different time periods, providing the quality of the sunlight does not vary greatly.

Studies on the effect of shading upon plant growth, by Lubimenko ${ }^{26}$ Combes, ${ }^{27}$ Rose, ${ }^{28}$ and others indicate that moderate variations in light intensity may be accompanied by very great differences in plant growth. In most

25 Richter, A., Etude sur la photosynthèse et sur l' absorption par la feuille verte. 1Rev. gen. Bot. 14: 151-169, 211-215. 1902 .

36 Lubimenko, W., Production de la substauce seehe et de la chlorophyll chez les vegetaux superieures aux differents intensités lumineuses. Ann. Sci. Nat. Bot. IX, 7:321-415. 1908.

27 Combes, R. La determination des intensités lumineuses optima par les vegetaux au divers stades de developpenent. Ann. Sei. Nat. Bot. IX, 11: 74-254. 1910.

${ }_{28}$ liose, Edmond, L'energie assimilatrice ehez les plantes. Ann. Sei. Nat. Bot. IX, 17: 1-110. 1913. 


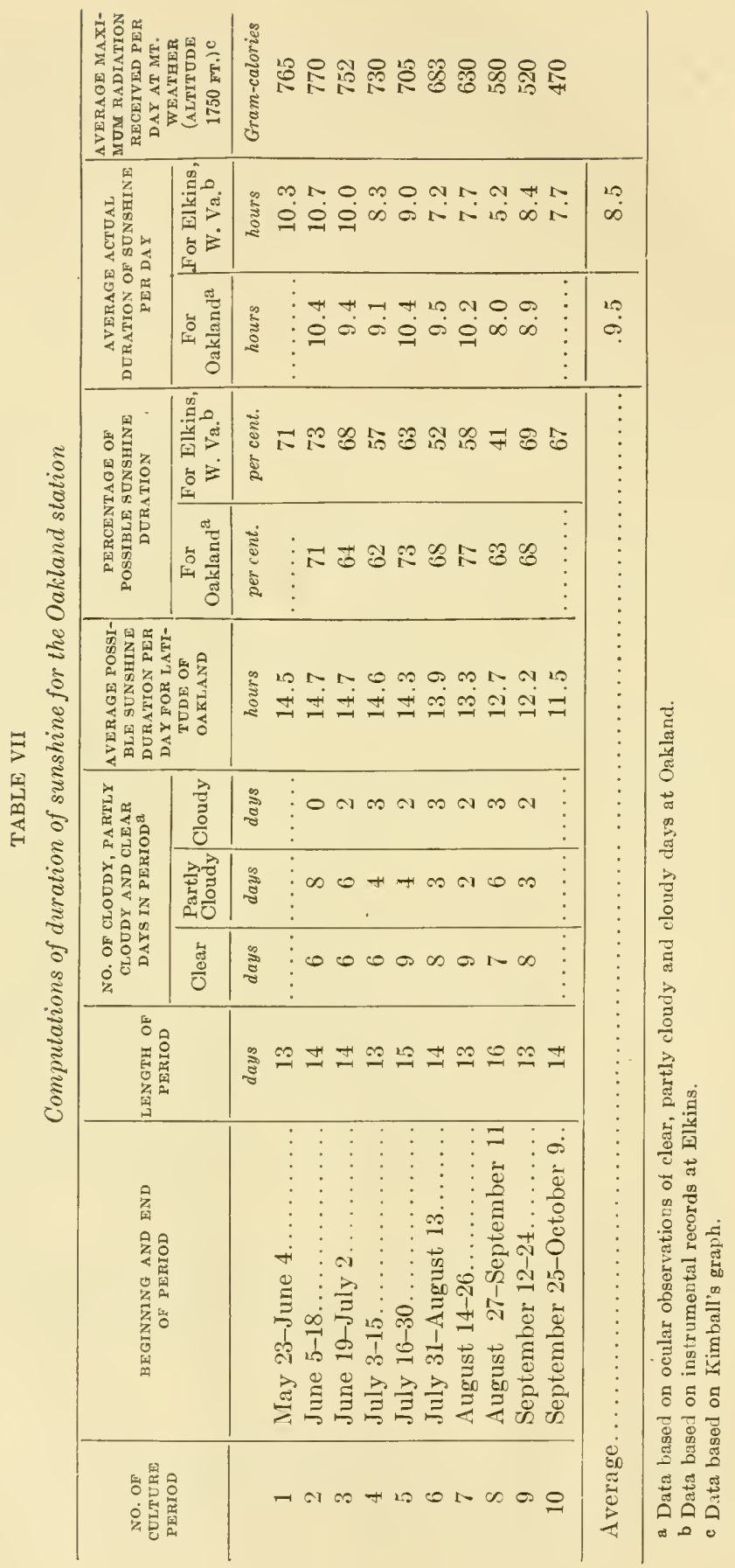




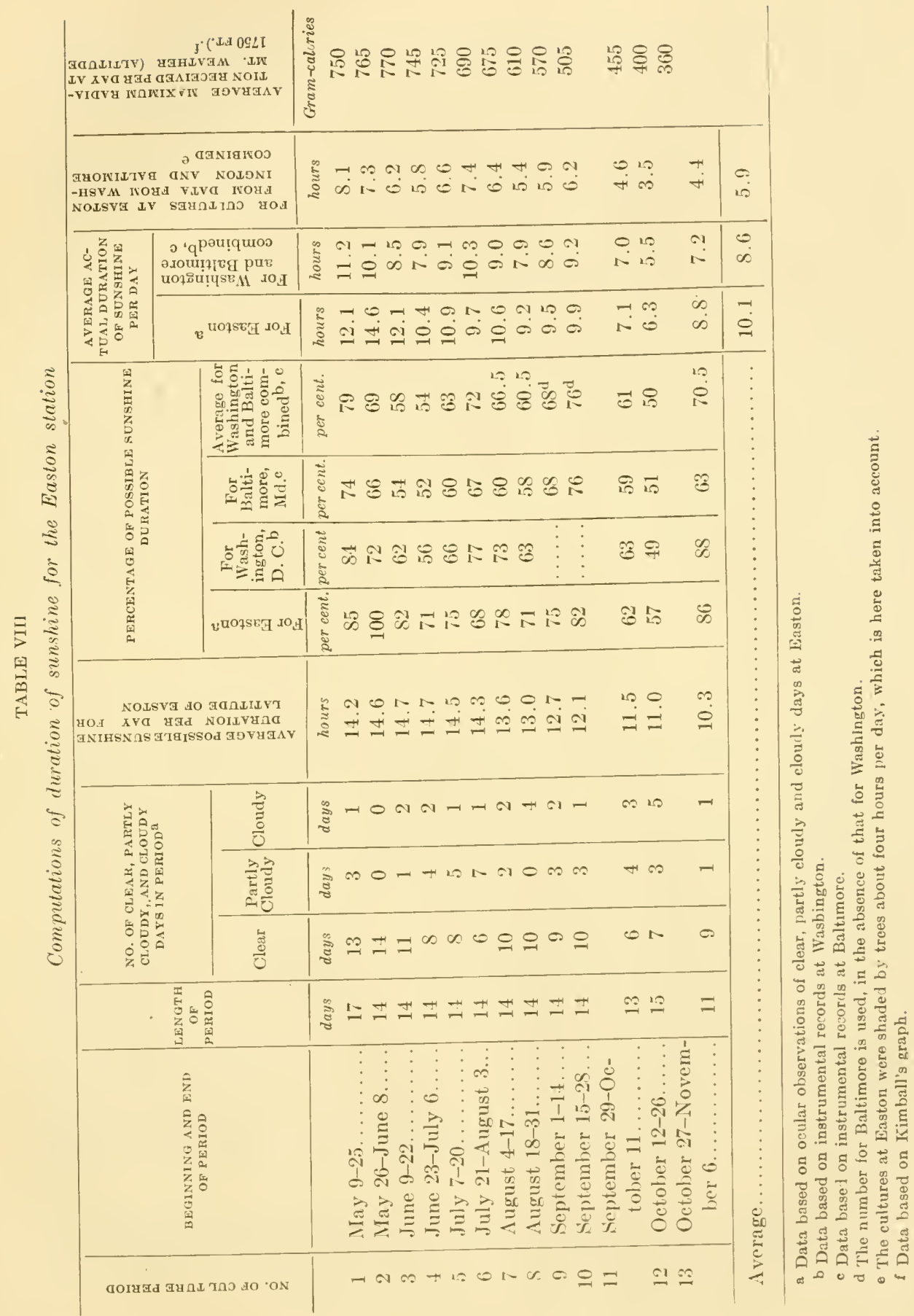


of these experiments, however, the conditions affecting the evaporating power of the air and the transpiration rates were not precisely enough measured or defined to make it certain that the differences in growth attributed to differences in light were indeed entirely light affects.

In addition to the sunshine records, the seasonal march of solar radiation in general is shown in the final columns of tables VII and VIII. These data are from Kimball's ${ }^{29}$ graph of the maximum daily amount of radiation received upon a horizontal surface during each decade of the year, and is based on three years' record at Mt. Weather, Va. The latter station has an altitude of about $520 \mathrm{~m}$. (1750 ft.) above sea-level, and is therefore fairly comparable to Oakland, Md. The main value of this Mt. Weather record for the present purpose, however, is to show the seasonal march of the average maximum intensity per day, of solar radiation, for each culture period, for this general region. Thus, the average daily maximum radiation for culture period 3 for Easton appears to be more than twice as great as that for period 13 for the same station, and a similar relation holds, of course, for Oakland. These differences in the extreme values of total solar radiation per day are partly determined by the actual differences in radiation intensity at different seasons of the year, and partly by corresponding differences in the lengths of the daily periods of daylight.

\section{PRESENTATION OF WEATHER DATA}

All of the weather data here considered have been computed as summations or averages for periods corresponding to the first two weeks (approximately) of each full culture period, and also for each entire culture period of about four weeks, and the resulting values are given in tables IX-XII, which have already been mentioned. Tables IX and X refer to Oakland, tables XI and XII to Easton. Tables IX and XI show the data for the first two weeks (approximately) of each full culture period. The first line gives the serial numbers of the cultures, as heretofore used. Lines 2 and 3 give the dates of the beginning and end of each two-week period. Line 6 gives the direct summation of the daily mean temperature values in terms of degrees Fahrenheit above $40^{\circ} \mathrm{F}$, for each period. The average daily mean effective temperature (line 7 ) is derived by dividing each number in line 6 by the number of days in the period (line 4). Line 8 shows the average daily range of temperature for each two-week period, this being the difference between the average daily minimum and the average daily maximum.

29 Kimball, Herbert $H$., The total radiation received on a horizontal surface from the sun and sky at Mount Weather. Monthly Weather Rev, 42: 4i4-457. 1914. (See especially fig. 8, page 484.) 
Lines 9 to 12 give temperature efficiency indices for each period, computed in three different ways, according to the general method proposed by Livingston and Livingston, employing the chemical temperature cocfficient. (1) To obtain the values given in line 9, the averge daily mean temperature for the two-week period in question was first found, and then the efficiency index corresponding to this value was obtained directly from the table given by Livingston and Livingston ${ }^{30}$ (page 366). To give the efficiency indices for the entire period (line 10), each number given in line 9 was multiplied by the corresponding number of days (line 4). (2) To obtain the temperature efficiency values given in line 11, the mean temperature for each day of the period in question was found by areraging its maximum and minimum. The efficiency index corresponding to each of these daily means was then found from the Livingston and Livingston table, and these indices were finally summed. (3) The values given in line 12 were derived in still another way. The efficiency index corresponding to the maximum and that corresponding to the minimum were found, from the table just mentioned, for each day of the period considered, and these two indices were averaged. The average daily efficiency indices thus obtaine:1, were finally sumned.

Lines 13 and 15 exhibit the total amount of rainfall for each two-week period, in centimeters and in inches. Line 14 gives the average daily rainfall for the period, in centimeters. Line 16 shows the total evaporation for the period, in cubic centimeters, from the cylindrical porous cup atmometer, the readings having been first corrected to the Livingston cylindrical standard, while line 17 gives the average daily evaporation for the period. Line 18 gives the value of the rainfall-evaporation ratio for each period, this being obtained by dividing each number in line 13 by the corresponding one in line 16. Line 19 indicates the total number of hours of sunshine recorded by the Marvin sunshine recorder at Elkins, IV. Va. (representing Oakland) or the average of the similar records obtained at Baltinore and at Washington (representing Easton), for each period. Each value in line 19 divided by the number of days in the period (line 4) gives the corresponding value in line 20 , which is the average daily duration of sunshine for the period.

Tables $\mathrm{X}$ and XII have the same form as tables IX and XI, and all of the description just given applies also to these, excepting that the data of tables $\mathrm{X}$ and XII refer to the full culture periods of approximately 4 weeks instead of to the first two-week portion of each full period.

\footnotetext{
30 These efficiency indices were derived by the authors just mentioned, by considering the growth rate as unity at $40^{\circ} \mathrm{F}$. and supposing that this rate doubles with each rise of $18^{\circ}$ above $40^{\circ} \mathrm{F}$.
} 
Forman T. McLlean

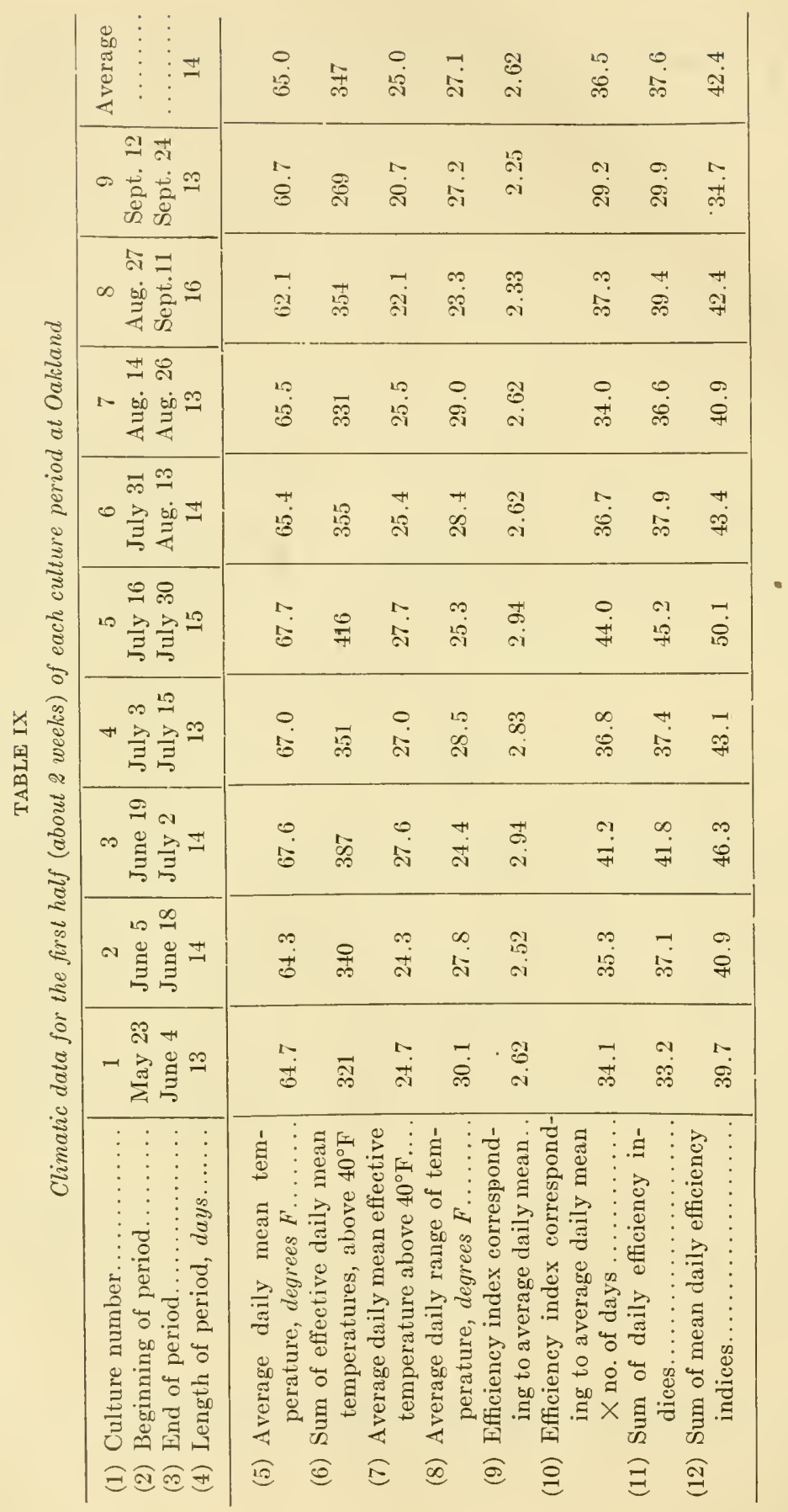




\begin{tabular}{|c|c|c|c|c|}
\hline 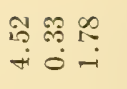 & $\underset{i}{\vec{i}}$ & $\begin{array}{l}19 \\
129\end{array}$ & 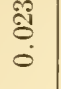 & $\stackrel{\infty}{\Rightarrow} \quad \infty$ \\
\hline 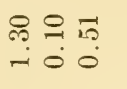 & $\stackrel{19}{ \pm 1}$ & $\stackrel{? !}{=}$ & 0 & $\stackrel{\overrightarrow{4}}{\circ}$ \\
\hline 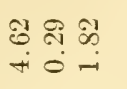 & $\stackrel{9}{\sharp}$ & $\ddot{\infty}$ & $\dot{0}$ & $\begin{array}{lll}2 & 0 \\
& 10\end{array}$ \\
\hline 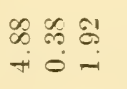 & 虽 & $\stackrel{9}{=}$ & 0 & $\stackrel{1}{1}$ \\
\hline 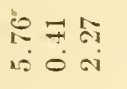 & $\overrightarrow{\text { ते }}$ & $\stackrel{0}{ \pm}$ & $\begin{array}{l}0 \\
0 \\
0\end{array}$ & $\stackrel{i}{i}$ \\
\hline 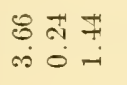 & $\overrightarrow{\mathrm{S}}$ & $\stackrel{\theta}{\dot{\theta}}$ & $\dot{0}$ & $\stackrel{0}{9} \stackrel{0}{9}$ \\
\hline 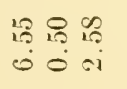 & 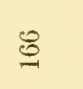 & $\stackrel{\infty}{\infty}$ & $\begin{array}{l}\overline{0} \\
0\end{array}$ & $\stackrel{\mathscr{c}}{=} \quad \stackrel{\infty}{=}$ \\
\hline 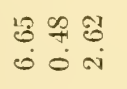 & ผิ & 92 & $\begin{array}{l}\text { है } \\
\stackrel{0}{0}\end{array}$ & $\stackrel{\circ}{\stackrel{0}{\varrho}}$ \\
\hline 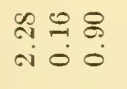 & ले & is & $\begin{array}{l}8 \\
0 \\
0\end{array}$ & $\stackrel{9}{\because}$ \\
\hline $\begin{array}{l}8.50 \\
100 \\
0\end{array}$ & స్ & $\begin{array}{l}\infty \\
+ \\
+1\end{array}$ & $\begin{array}{l}0 \\
0 \\
0\end{array}$ & 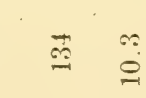 \\
\hline 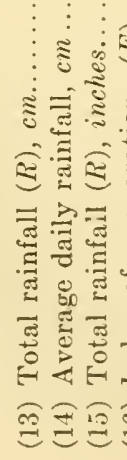 & $\begin{array}{l}\vdots \\
\vdots \\
\vdots \\
\vdots \\
\vdots \\
\vdots \\
\vdots \\
\vdots \\
\vdots \\
\vdots \\
\vdots \\
\vdots \\
\vdots \\
0 \\
0\end{array}$ & 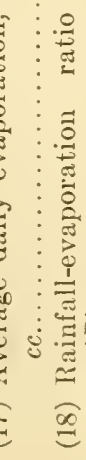 & $\begin{array}{c}\vdots \\
\vdots \\
\vdots \\
\vdots \\
\vdots \\
\vdots\end{array}$ & 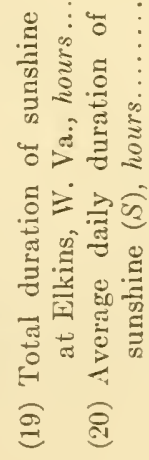 \\
\hline
\end{tabular}




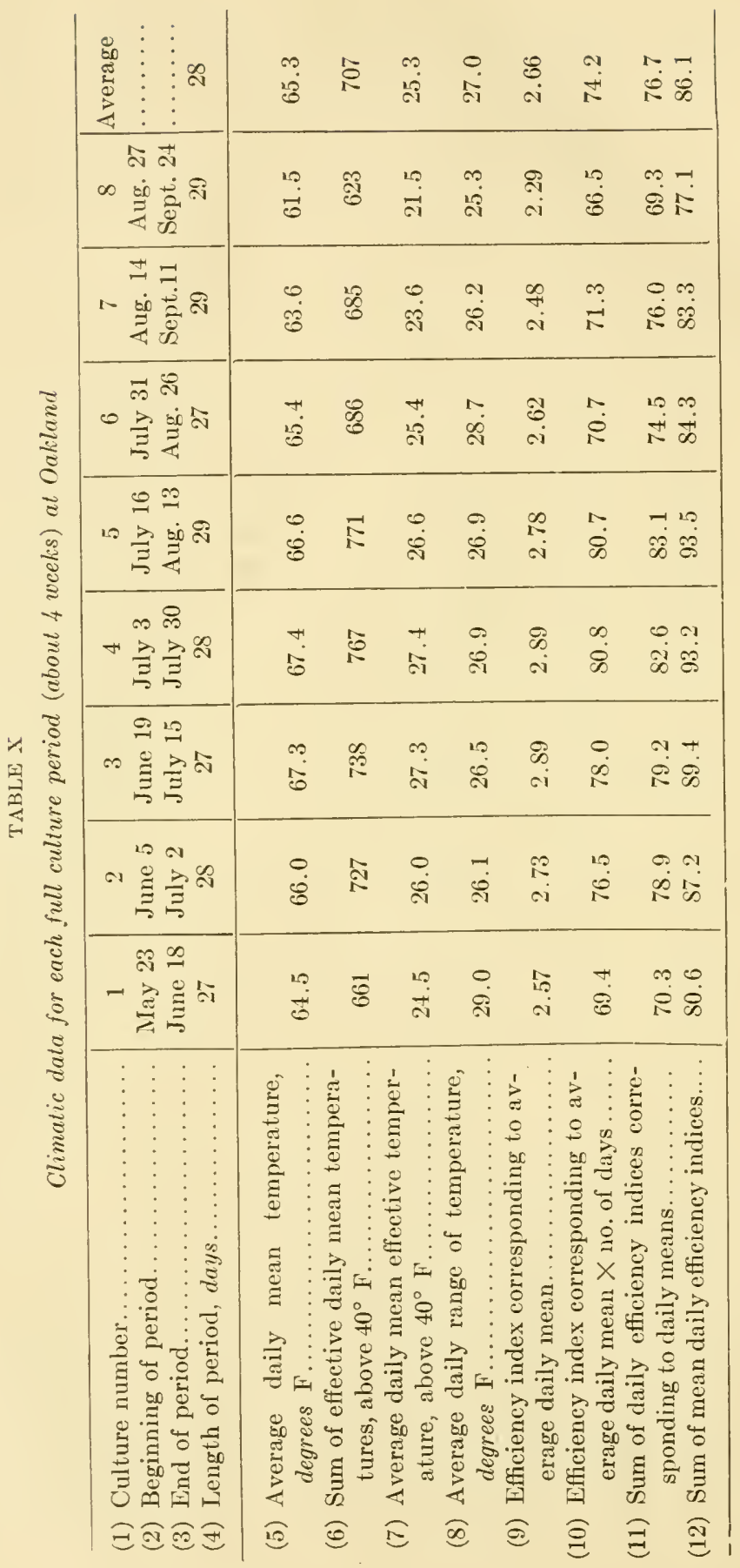


Climatic Conditions in Maryland

\begin{tabular}{|c|c|}
\hline 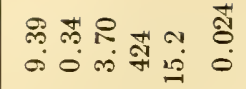 & ศึ \\
\hline 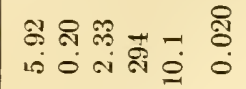 & $\stackrel{\infty}{\doteq}$ \\
\hline 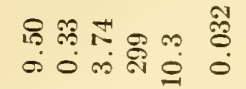 & $\stackrel{\mathscr{D}}{\infty}$ \\
\hline 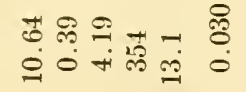 & ชิ \\
\hline 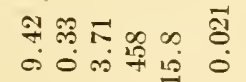 & ผึ \\
\hline 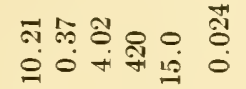 & $\underset{N}{N} \infty$ \\
\hline 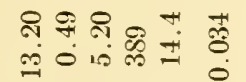 & 虽 \\
\hline 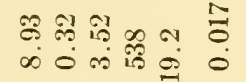 & $\overrightarrow{\overparen{S}}$ \\
\hline 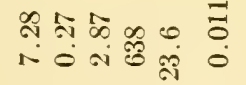 & $\overrightarrow{\Phi_{N}}$ \\
\hline 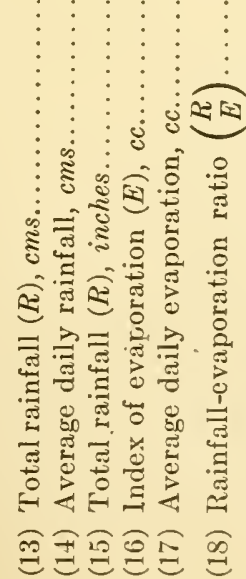 & 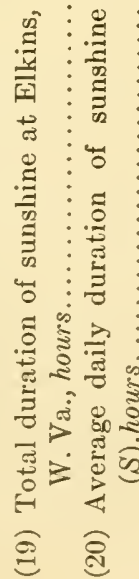 \\
\hline
\end{tabular}

PHYSIOLOGICAL RESEARCHES, VOL. 2, No. 4, SERIAL NO. 14, FEBRUARY, 1917 


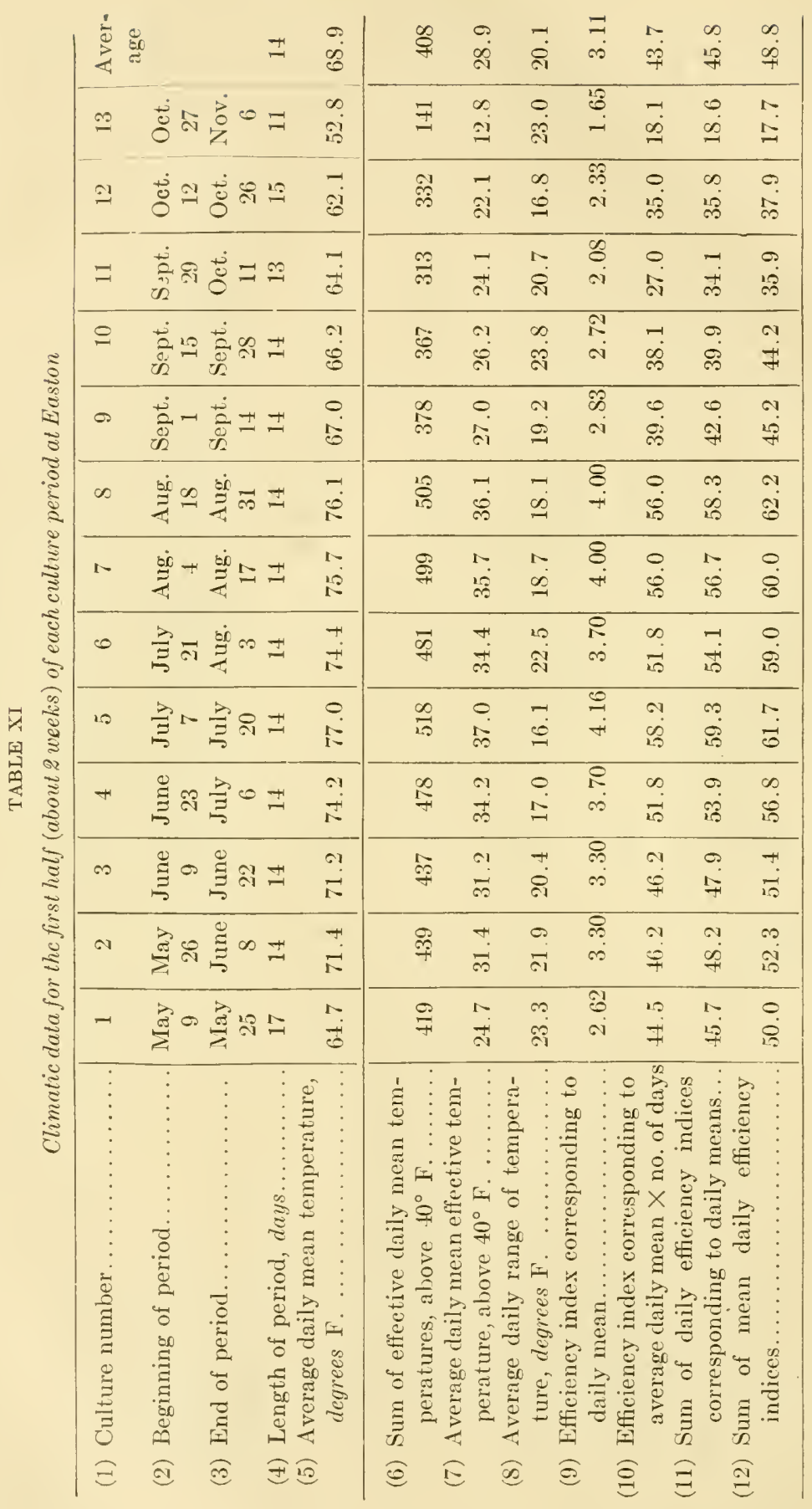




\begin{tabular}{|c|c|c|}
\hline 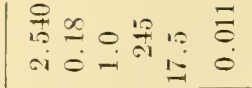 & ت: & $\begin{array}{l}\infty \\
10\end{array}$ \\
\hline 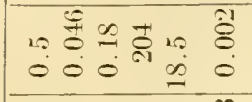 & $\stackrel{f}{f}$ & + \\
\hline 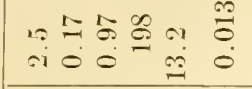 & $\hat{i}$ & $\therefore$ \\
\hline 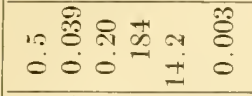 & 8 & $\stackrel{0}{+}$ \\
\hline 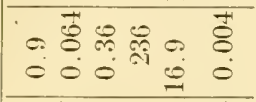 & 冓 & $\stackrel{?}{e}$ \\
\hline 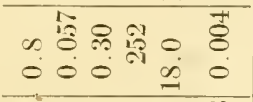 & $\mathscr{8}$ & is \\
\hline 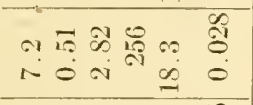 & $R$ & is \\
\hline 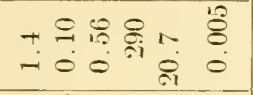 & 8 & H. \\
\hline 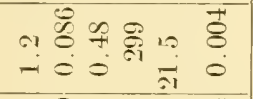 & $\overrightarrow{\underline{O}}$ & $\stackrel{+}{N}$ \\
\hline 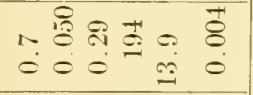 & $\hat{\mathrm{g}}$ & $\begin{array}{l}0 \\
0\end{array}$ \\
\hline 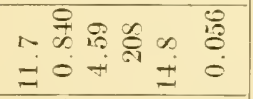 & $\vec{\infty}$ & $\begin{array}{l}\infty \\
10\end{array}$ \\
\hline 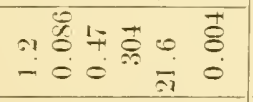 & $\frac{1}{x}$ & $\ddot{0}$ \\
\hline 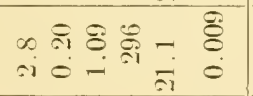 & $\hat{\stackrel{\theta}{\mathrm{\theta}}}$ & $\because$ \\
\hline 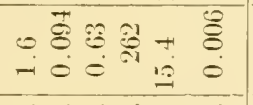 & $\stackrel{\sigma}{\cong}$ & $\vec{\infty}$ \\
\hline 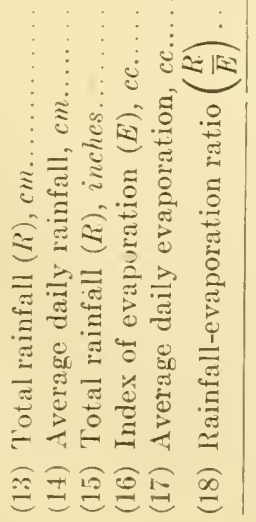 & 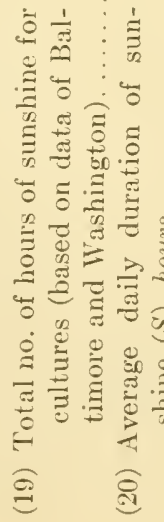 & 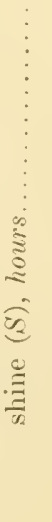 \\
\hline
\end{tabular}




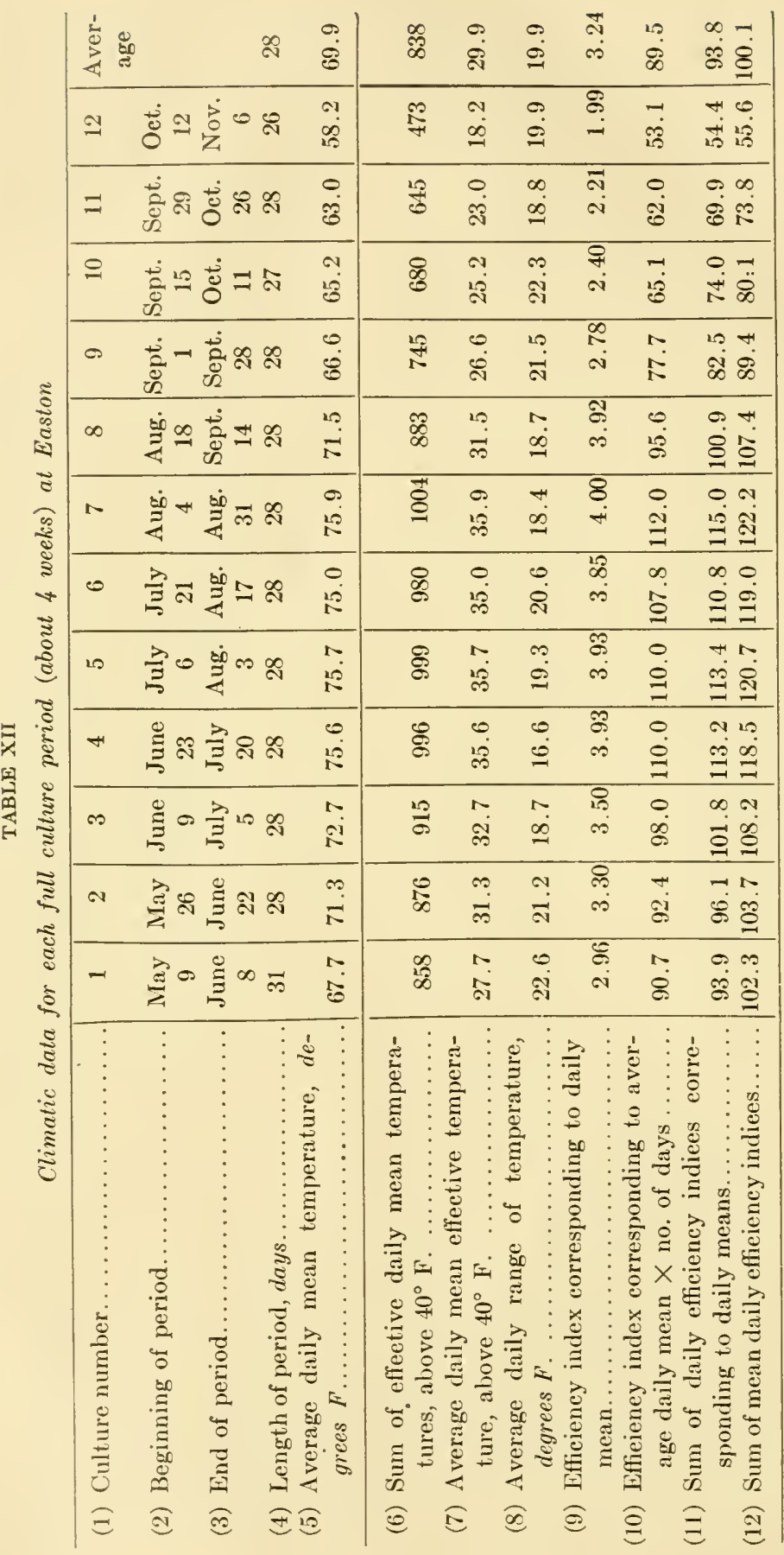




\begin{tabular}{|c|c|}
\hline 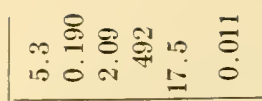 & $\ddot{0} \quad 0$ \\
\hline 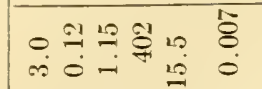 & E $\quad 0$ \\
\hline 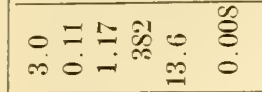 & $\stackrel{20}{=}$ \\
\hline 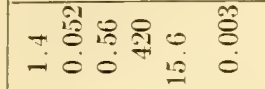 & 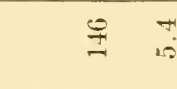 \\
\hline 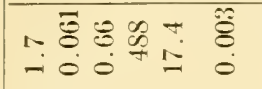 & $E \quad$ \\
\hline 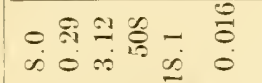 & 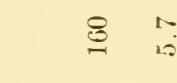 \\
\hline 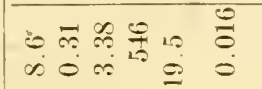 & $\stackrel{13}{\cong} \quad 0$ \\
\hline 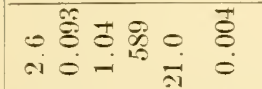 & $\cong \quad 0$ \\
\hline 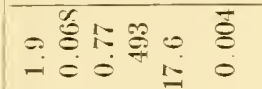 & $\mathscr{E} \quad 0$ \\
\hline 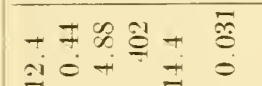 & E \\
\hline 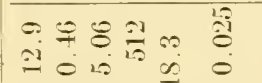 & $\begin{array}{ll}\infty & 0 \\
0 & 0\end{array}$ \\
\hline$=\frac{\pi}{0}=\frac{8}{8}$ & $\Xi \infty$ \\
\hline 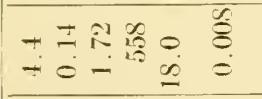 & 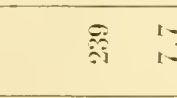 \\
\hline 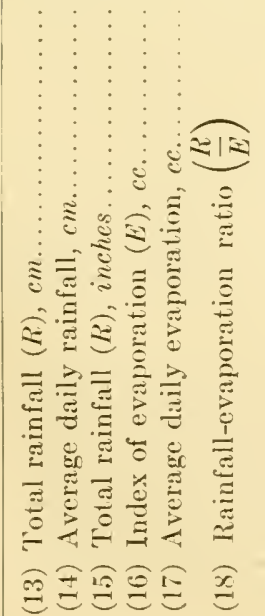 & 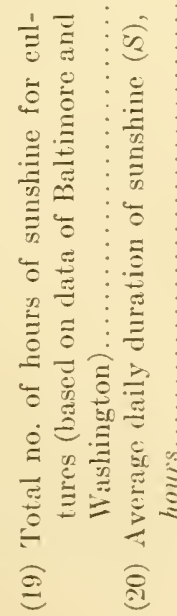 \\
\hline
\end{tabular}




\section{DISCUSSION}

The Oakland and Easton stations, as has been stated, were selected for comparative study in this paper because they may be taken to represent the extremes of the climatic conditions encountered in the Maryland area. In the discussion that follows, the various climatic features measured for these two stations during the summer of 1914 , will be considered as environmental conditions presumably affecting the growth of the corresponding culture plants, and these paragraphs will present comparisons of plant features with climatic conditions for Oakland and for Easton, as well as comparisons of the Oakland data with those of the other station. An attempt will be made to bring out some of the relations that obtained for the different observation periods at each station, between the soy bean plants and the concomitant and supposedly controlling conditions of their surroundings.

\section{THE FROSTLESS SEASON}

It has already been noted that the season of active plant growth proved to be much shorter at Oakland than at Easton. The frostless season at Oakland, for 1914, began after a severe frost on June 17, and ended with another severe frost on September 28. Thus the duration of this season was 103 days for Oakland. The experiments with which this paper deals were not begun at Easton until May 8, so that the last severe spring frost was not actually encountered at that station, but the records of the local observer show that this probably occurred April 11. The first killing frost in autuinn occurred at Easton on November 7 , so that the length of the frostless season of 1914 was approximately 210 days for that station. At Oakland the frosts mentioned were severe enough to kill soy bean seedlings in the general region, and the autumn frost actually did kill the plants in the experimental cultures. The same statement holds regarding the season at Easton, except that, since the last spring frost was not actually experienced by the cultures, its severity is merely inferred. The difference between the lengths of the frostless seasons for Easton and Oakland, as here indicated (107 days), appears to be greater than is usual; as is clear from Fassig's ${ }^{31}$ data, the normal length of the frostless season for Easton is 201 days and that for Oakland is 134 days, the normal difference being thus only 67 days.

31 Fassig, Oliver L., The period of safe plant growth in Maryland and Delaware. Monthly Weather Rev 42: 152-158. 1914 


\section{COMPARISONS BETWEEN THE PLANT GROWTH VALUES AND CLIMATIC \\ INDICES FOR OAKLAND WITH THOSE FOR EASTON, FOR THE \\ ENTIRE PERIOD OF OBSERVATIONS}

Besides the length of the growing season, many other climatic characters are of course influential in determining plant growth, and some of these will now be considered, in relation to the corresponding growth characters exhibited by the culture plants here dealt with.

The comparative efficiencies of the climatic conditions, as a whole, expressed in terms of the growth of the soy-bean plants here employed, for the two stations and for the entire period of experimentation, are presented in the last column in tables I-IV. All of these are expressed both as average rates per day and as rates per period, excepting those for length and width of mature leaves ( $l$ and $w$ ), which are given only as rates per period. These measirements of mature leaves were obtained in order to determine the influence of the surroundings on the size attained by the leaves when mature, and these data were therefore not related to the rate of leaf expansion; they refer simply to the limit to this expansion set by the surroundings. Therefore average daily rates were not obtained in these two cases. On the other hand, those measurements of leaf length and width that were made for all leaves (whether mature or not) and that furnish the data for the leaf-products $(P)$, require expression as mean daily rates. These leafproducts proved to be approximate measurements of total leaf surface per plant, developed during their respective periods. The data for twoweek periods do not, of course, include actual measurements of leaf area, but in the case of the four-week periods the derived leaf-products and the actually measured leaf areas are both available for comparison (tables II and IV, lines 12 and 14). It is seen at onee that these two series of values vary in the same direction across the tables. In order to investigate this parallelism each leaf-product in line 12 was divided by the corresponding leaf area in line 14 , thus giving the ratio $\frac{P}{A}$ (line 18). If the leaf-products were always actually proportional to the corresponding leaf area, this ratio would have a constant value, and the actual values are scen, indeed, to be nearly constant. The average value of $\frac{P}{A}$ in table II, for Oakland, is 1.34 and the greatest plus and minus variations from this value are 7.4 and 5.2 per cent., respectively. Similarly this ratio average in table $I V$, for Easton, is 1.32 , with maximum plus and minus variations of 7.5 and 6.1 per cent., respectively. It is therefore very clear that the leaf-product values (derived by measurements that do not involve any injury to the leaves) are to be considered as almost truly proportional to the leaf areas (which cannot be readily obtained without the removal of 
the leaves from the plants). Since this conclusion holds so well in the cases where both leaf-products and leaf areas are available, it is probably safe to assume a similar relation in the other cases. Therefore, the leaf-products are here employed as approximate measures of leaf area both for the twoweek and for the four-week periods.

The average daily rates of plant growth for the whole season of these studies (measured by the various criteria) are given for both stations in columns 3 and 4 of table XIII, and these columns also include the data of length and width of mature leaves, all data being taken without change from the last column of tables I-IV. Column 5 of table XIII presents the ratios obtained by dividing each value for Oakland by the corresponding value for Easton. These ratios of the plant measurements thus express each average daily value for Oakland in terms of the corresponding average for Easton, and bring out the relations between the climatic conditions for the two stations, as indicated by the culture plants.

The daily averages of the various iveather data for the entire experimental season are also given, in columns 7 and 8 of table XIII, only one of the three temperature efficiency indices (the one corresponding to the daily mean, tables $\mathrm{X}$ and XII, lines 10) being given here. Column 9 gives the ratios obtained by dividing each climatic value for Oakland by the corresponding value for Easton. Thus, these climatic ratios express each one of the various climatic values for Oakland in terms of the corresponding one for Easton, as in the case of the growth values.

The most evident feature brought out by the plant data given in table XIII is that the values for Easton are generally greater than t: e corresponding ones for Oakland. This is strictly true of the values based on the shorter observation periods (plants about 2 weeks old from seed) and it is true of those based on the full culture periods (plants about 4 weeks old from seed) excepting in the case of the mean daily rate of increase in the total number of leaves per plant and in that of average length of mature leaves. The greatest difference between the average daily growth rates for the two stations occurs in the case of the rate of increase in the average of the products of leaf length by leaf width (leaf-product, $P$ ) for the shorter periods. For these younger plants the ratio of this rate for Oakland to that for Easton is 0.73 . The greatest difference in these values based upon plants about 4 weeks old, for the two stations, is shown by the rates of increase in leaf area, dry weight of tops, and leaf-product, the ratios of these rates for Oakland to those for Easton being 0.80, 0.86 and 0.89, respectively. The rate of increase in leaf area, measured either directly (by the planimeter) or approximately (by means of the leaf-product), and also the rate of increase in dry weight of tops, since these rates exhibit the greatest differences between the two stations, may be considered as of probable value for comparing the effectiveness of the climatic conditions for the growth of the culture plants at one station with their effectiveness at the other. 
The differences in the average daily values obtained for the climatic conditions, as here recorded for the two stations (table XIII, columns 6 to 9), are quite as great as or greater than the differences in the growth values. In comparing these it is to be remembered that the averages of the climatic data for the 2 -week and 4 -week periods are both computed from the same series of measurements, covering practically the same periods of time (these periods corresponding to the periods for which the plant data are computed), so that the average values derived from the two lengths of period are very nearly the same in this case, and they may be generally considered as practically identical for the purpose of comparison with the daily averages of the plant data for the two stations. The daily averages of effective temperature (temperature in excess of $40^{\circ} \mathrm{F}$.) and of the efficiency index (derived from the chemical coefficient) are all about 84 per cent. as great for Oakland as they are for Easton, and a similar relation holds for the mean daily rate of evaporation, this rate being about 88 per cent. as great for Oakland as for Easton. The mean daily rainfall on the contrary is about 80 per cent. greater for Oakland than for Easton, and the rainfall-evaporation ratio for Oakland is more than double that for the other station. Similarly, the average daily duration of sunshine is about 43 per cent. greater for Oakland. Finally, the Easton station shows a higher average daily mean temperature, by about $5^{\circ} \mathrm{F}$, but a smaller average daily range of temperature by about the same amount.

It appears that the average of the climatic conditions experienced by the successive cultures at Easton was more favorable for increase in leaf surface and in dry weight of tops (for both the 2-week and the 4-week periods) than was the corresponding average experienced by the cultures at Oakland. Excepting the average daily mean temperature, all of the major groups of environmental factors here considered were very different for the two stations, and the daily averages given in table XIII do notinrlicate which factor, or group of factors, may have been most influential in bringing about these differences in the growth rates. Of course it is possible that other factors than those considered in this study may also have been influential in producing the recorded differences in plant growth here brought out. Some information as to the relative influences exerted by the different groups of elimatic conditions that have been instrumentally measured will appear below. 
Forman T. Mclean

\begin{tabular}{|c|c|c|c|c|c|c|c|c|c|c|}
\hline & & 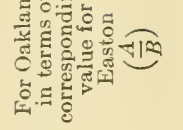 & $\begin{array}{l}\vec{b} \\
0\end{array}$ & t. & 然 & $\begin{array}{l}\vec{\infty} \\
0\end{array}$ & $\mathscr{\infty}$ & $\begin{array}{l}\infty \\
0 \\
0\end{array}$ & $\begin{array}{c}8 \\
\text { ci }\end{array}$ & $\stackrel{19}{-2}$ \\
\hline & $\overbrace{g}^{n}$ & 施 & $\begin{array}{l}0 \\
\dot{0}\end{array}$ & $\underset{\dot{d}}{\infty}$ & $\overrightarrow{\text { जे }}$ & $\Rightarrow$ & $\underset{-1}{\infty}$ & $\stackrel{?}{\simeq}$ & $\begin{array}{l}\ddot{1} \\
0 \\
0\end{array}$ & is \\
\hline 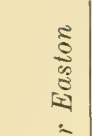 & $\begin{array}{l}0 \\
0 \\
0 \\
0 \\
0 \\
0 \\
\end{array}$ & 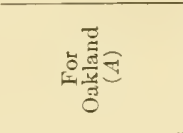 & : & 豈 & 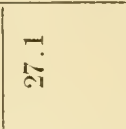 & $\begin{array}{l}\underbrace{1}_{0} \\
\text { ci }\end{array}$ & \% & $\begin{array}{l}120 \\
20\end{array}$ & हैं & $\begin{array}{l}10 . \\
\infty\end{array}$ \\
\hline 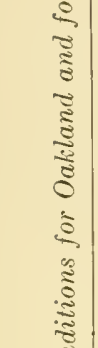 & 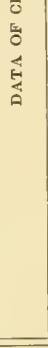 & & 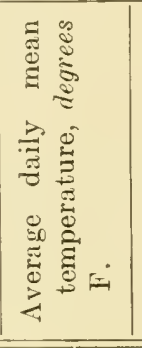 & 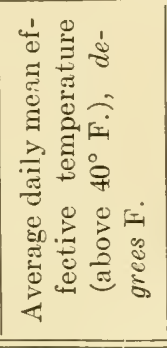 & 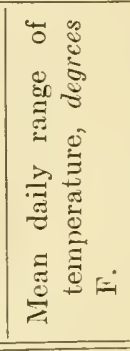 & 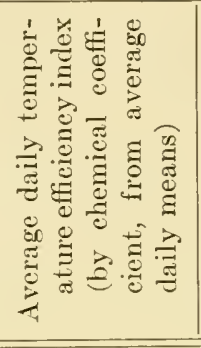 & 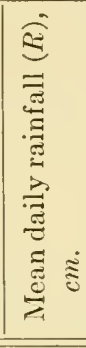 & 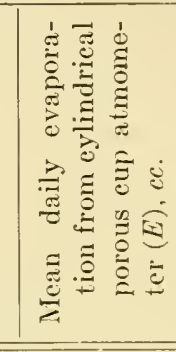 & 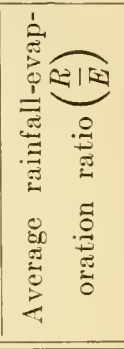 & 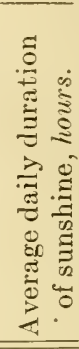 \\
\hline 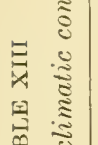 & & 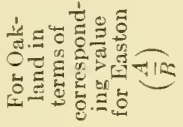 & $\begin{array}{l}\infty \\
\infty \\
0\end{array}$ & $\begin{array}{l}\infty \\
\infty \\
0\end{array}$ & & : & & $\begin{array}{l}\infty \\
\infty \\
0 \\
0\end{array}$ & $\stackrel{P}{\stackrel{0}{0}}$ & \\
\hline 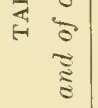 & & 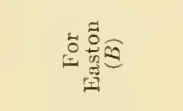 & 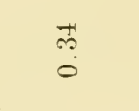 & $\stackrel{0}{\ddot{0}}$ & & $\ddot{b}$ & & $\vec{i}$ & $\stackrel{\infty}{-}$ & \\
\hline 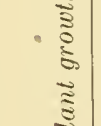 & 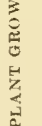 & 咅引 & $\stackrel{\leftrightarrow}{\circ}$ & $\stackrel{J}{ \pm}$ & & $\stackrel{H}{\Delta}$ & & $\overrightarrow{c i}$ & $\begin{array}{l}\mathscr{D} \\
0 \\
0\end{array}$ & \\
\hline 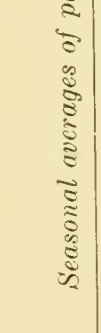 & 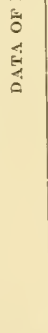 & & 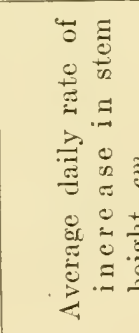 & 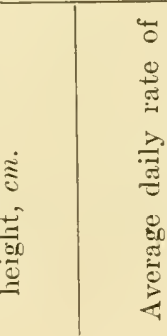 & 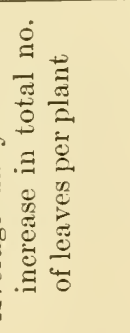 & 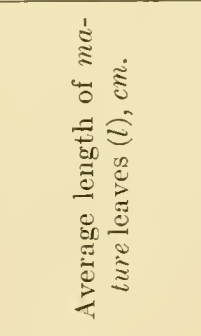 & & 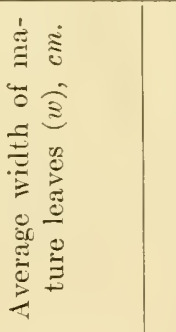 & 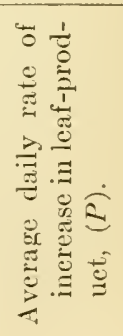 & \\
\hline & & & & & & 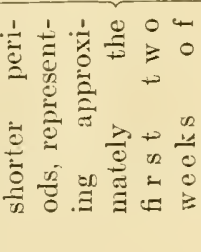 & 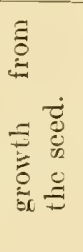 & & & \\
\hline
\end{tabular}


Chimatic Conditions in Maryland

\begin{tabular}{|c|c|c|c|c|c|c|c|}
\hline$\stackrel{8}{:}$ & $\begin{array}{l}18 \\
0 \\
0\end{array}$ & ஸొ & $\begin{array}{l}a \\
\infty \\
0 \\
0\end{array}$ & $\stackrel{R}{-}$ & 占 & $\underset{i i}{\infty}$ & $\stackrel{\text { Iै }}{\rightarrow-1}$ \\
\hline$\stackrel{8}{8}$ & $\stackrel{0}{8}$ & $\stackrel{\oplus}{\stackrel{\theta}{\sim}}$ & $\begin{array}{l}\overrightarrow{a t} \\
i\end{array}$ & $\stackrel{0}{0}$ & $\stackrel{8}{12}$ & $\begin{array}{l}\vec{\sigma} \\
\dot{0}\end{array}$ & $\begin{array}{l}8 \\
0\end{array}$ \\
\hline$\dddot{3}$ & $\stackrel{\leftrightarrow}{a}$ & $\begin{array}{l}0 \\
\text { Si }\end{array}$ & $\begin{array}{c}8 \\
\text {-i }\end{array}$ & $\begin{array}{l}+ \\
0\end{array}$ & $\begin{array}{l}01 \\
13 \\
21\end{array}$ & $\begin{array}{l}\overrightarrow{1} \\
0 \\
0\end{array}$ & is \\
\hline 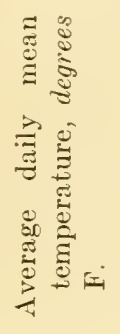 & 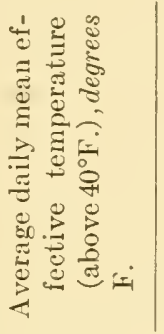 & 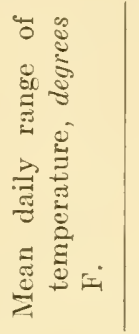 & 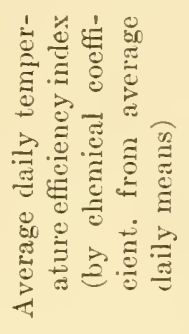 & 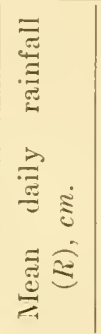 & 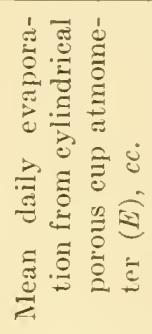 & 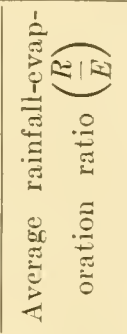 & 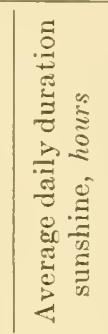 \\
\hline$\stackrel{\mathscr{B}}{\circ}$ & $\underset{-i}{8}$ & $\underset{-i}{8}$ & $\stackrel{20}{\mathrm{C}}$ & $\begin{array}{l}\mathscr{W} \\
0 \\
0\end{array}$ & $\begin{array}{l}8 \\
0 \\
0\end{array}$ & & $\begin{array}{l}\infty \\
\infty \\
0\end{array}$ \\
\hline$\stackrel{\vec{N}}{0}$ & $\begin{array}{l}= \\
0\end{array}$ & $\stackrel{0}{i}$ & 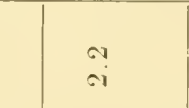 & $\stackrel{\leftrightarrow}{-}$ & s. & & $\begin{array}{l}\overrightarrow{3} \\
8 \\
0 \\
0\end{array}$ \\
\hline$\stackrel{\hat{i}}{\hat{i}}$ & $\stackrel{1}{0}$ & $\stackrel{\theta}{\Delta i}$ & $\overrightarrow{a i}$ & $\cong$ & $\begin{array}{l}\mathscr{0} \\
\dot{0}\end{array}$ & & $\begin{array}{l}\frac{7}{8} \\
0 \\
0\end{array}$ \\
\hline 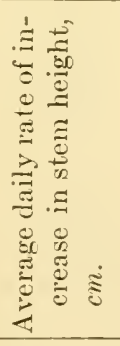 & 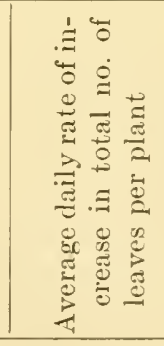 & 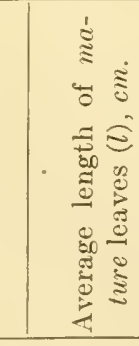 & 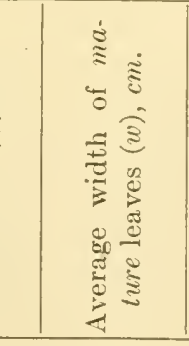 & 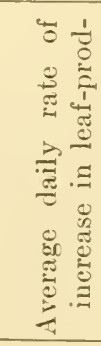 & 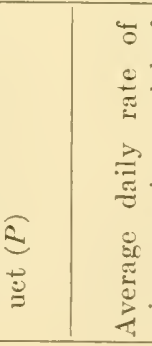 & 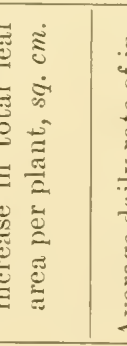 & 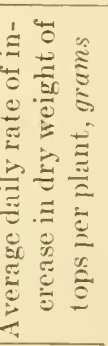 \\
\hline & & 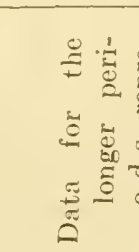 & 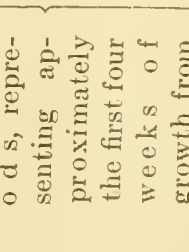 & & & & \\
\hline
\end{tabular}




\section{THE SEASONAL MARCH OF THE GROWTH RATES AND OF THE CLIMATIC CONDITIONS AT OAKLAND AND AT EASTON}

\section{INTRODUCTION}

Any season in any locality may be considered as made up of a series of relatively favorable and relatively unfavorable periods, all of which contribute to the sum total of the season's influence upon the magnitude of the final yield of plants. The ultimate influence of any given portion or periou of the growing season upon the final yield cannot generally be judged by the appearance of the plants at any time before the yield may actually be measured, for the thrift and rate of growth of a plant during any period of its development is a result of both present and past conditions. Thus, the rapid growth of a crop observed during a hot, dry week in midsummer, after a rain that terminated a drought period, is partly a result of the rain, which suppliec water for the expansion of the tissues, but it is probably almost as much due to conditions effective within the drought period, during which the plants may have elaborated reserve materials, an abundance of which is quite as requisite for rapid growth as is an abundance of water.

It is therefore necessary to study the seasonal march of each growth index and of each climatic index, as these vary throughout the season, for each station. These seasonal marches may be studied by employing the data recorded for either the two-week or the four-week periods; that is, by taking the first two weeks or the first four weeks of growth from the seed as the short period of observation for the plants. Since these two methods do not bring out the same points, both will be employed below. The following sections are devoted to a study of the seasonal marches of the various plant and climatic indices, (1) based on the two-week periods, and (2) based on the four-week periods.

\section{SEASONAL MARCHES BASED ON PERIODS OF APPROXIMATELY TWO WEEKS}

Plant growth rates. Inspection of tables I and III brings out the fact that the three kinds of leaf measurements (length and width of mature leaves and leaf-product) nearly agree in the direction of their variation from period to period for both stations. On this account only one of these leaf measurements, the leaf-product, which shows the greatest variations, will be studied here.

On the whole it appears that, of the five criteria of plant growth here employed, those of leaf-product and stem height show all of the essential points, and seem to be sufficient to exhibit the differences in growth rates from one period to the next. These alone will therefore receive attention.

The variations in these two growth rates, from period to period, may be 
best represented by means of the graphs of figure 8 which give the average daily values of leaf product and stem height for Easton and for Oakland. The ordinates of the points on these graphs are the successive daily averages per period of about two weeks, and the abscissas represent time and season, indicating the ends of the successive periods. Each graph thus shows the values of a single growth index as it varies through the season. The actual duration of the period for which any ordinate represents the average rate is indicated on the upper graph in each case (representing leaf

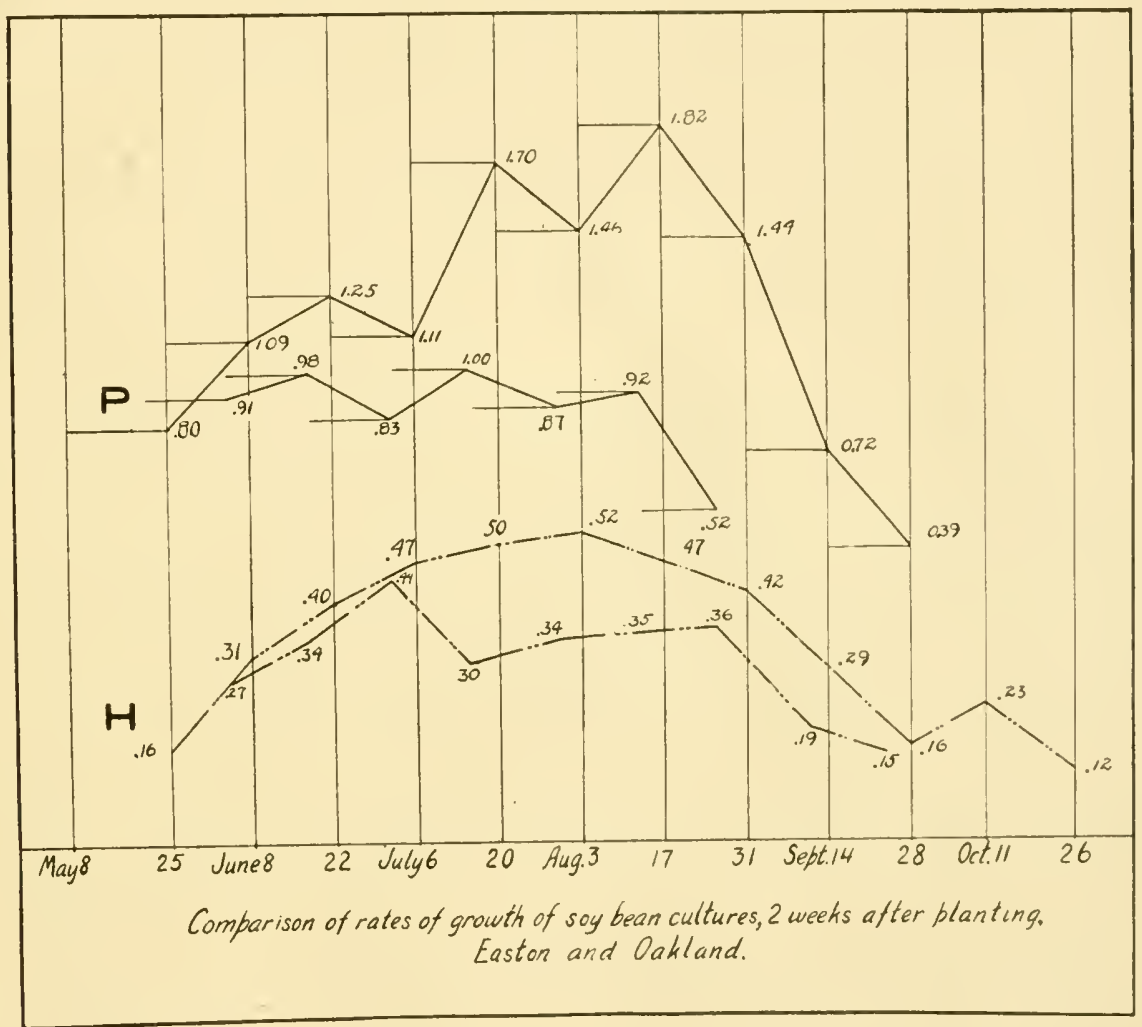

FIG. 8. $P$, leaf-product; $H$, stem height. The upper graph represents Easton, in each case.

product for Easton and for Oakland, respectively) by the length of the horizontai line extending to the left of the given point. The points on the corresponding graphs for stem height for Easton and for Oakland represent averages for the same time periods as are shown on the graphs of leaf-product.

Each of these graphs exhibits an evident seasonal march, the daily average per period increasing during a part of the season and then decreasing to low autumn values. This variation is more pronounced for Easton than for Oakland in both cases, which suggests that the seasonal changes in the 
environmental conditions were more pronounced at the former station. There is no apparent similarity of detail, however, between the seasonal marches of the two graphs for either leaf-product or stem height. The maximum in leaf-product for Easton occurs for the period from August 3 to 17, while that for Oakland occurs two weeks earlier. It must be noted, however, that a secondary maximum for Oakland occurs for the period from June 18 to July 2, and that this secondary maximum is almost as great as the other. It thus appears that the maximum rate of increase in leaf area may be expected to occur considerably earlier at Oakland than at Easton. In a similar manner the maximum rate of increase in height for Oakland is seen to occur about a month earlier than that for Easton.

This apparent difference in the seasonal position of the optimum period for the growth of these plants at the two stations, if it should prove to be a normal occurrence and if it be a characteristic difference between lowland and mountain (or inland and coastal) localities for this region, might suggest interesting explanations of various agricultural facts; for example, the common observation that grains and other crops ripen earlier at high altitudes than at lower ones. If the progress of the life cycles of such crops should be associated with the seasonal march of what might be termed the plant-producing power of the climate, and if the decline that follows the attainment of the maximum in the environmental tendency to produce vegetative growth should prove to be important in accelerating flower and fruit production, (both of which suppositions are quite possible, as far as is known at present), then the normal date at which this optimum is reached in any locality might be of great significance in determining the tendency of the climate of that region to produce early maturation of agricultural plants. Other more or less similar considerations might be mentioned, but no such questions as this can be answered without extensive investigation, continued through several years.

Climatic conditions. The three sets of daily climatic data available for this study of the two-week periods (those of temperature conditions, those of moisture conditions, and those of light conditions), as given in tables IX and XI, are set forth graphically in figures 9 and 10, the former referring to Oakland and the latter to Easton. To facilitate comparisons between the seasonal marches of these climatic averages and the corresponding marches of the two sets of values for average daily rates of plant growth for the first two weeks from the seed, the graphs of figure 8 are repeated in figures 9 and 10. Thus each of the latter figures comprises five graphs, all referring to the same station and to the same series of two-week periods. The upper three refer to climatic conditions and the lower two to the corresponding rates of plant growth. These five graphs are comparable, in each case, as to direction of slope and as to the position of minima and maxima, but it should be remarked that the lengths of their ordinates are not directly com- 
parable; the vertical scales employed are simply convenient ones and are quite arbitrary.

The graphs of temperature efficiency $(T)$ exhibit comparatively regular seasonal marches in both figures. The ordinate value of this graph for Oakland (figure 9) increases from 24.3, for the second two-week period, to 27.6 for the third period, continues high during periods 4 and 5 , and then decreases steadily to a final value of 20.7 for the minth period. The ordinate value of the corresponding graph of effective temperature for Easton (figure 10) continues to increase for a longer time in the early part of the seasoil. It attains a maximum value of 37.0 for the fifth period (which roughly corresponds to the fourth period at the other station), remains comparatively high (about 35.0) until the beginning of the ninth period (September 1), and then falls quite rapidly to a minimum of 12.8 for the thirteenth period, the last of the season. Thus the highest temperature efficiencies for Oakland oceurred about two or three weeks earlier than those for Easton, and the final decline of this climatic condition began a month earlier at Oakland than at the other station.

Another marked difference between the graphs of effective temperature for these two stations lies in the actual magnitudes of the various values. Not only are the values for corresponding time periods lower for Oakland than for Easton, but the differences between the highest and lowest value for Oakland is markedly smaller than the corresponding difference for Easton. The highest average effective temperature for Oakland is 27.7 , while the highest for Easton is 37.0, and the minimum value for Oakland is 20.7, while the minimum for Easton is 12.8. The minimum value for Easton, however, occurred about a month after the Oakland season had heen terminated by killing frost. From the above comparisons it appears that, in spite of the greater diurnal and interdiumal variations in temperature recorded for Oakland, the scasonal march of the mean daily effective temperature here shows smaller differences between the extremes for the season than are exhibited for Easton.

The graphs for sunshine $(S)$ show a general downward slope throughout the season, for both stations, but both of the graphs are very irregular.

The graphs for the rainfall-evaporation ratio $\left(\frac{K}{E}\right)$ are also irregular for both stations. Both show low values for the beginning and end of the season. That for Oakland shows a maximum for the two-week period ending July 30, and another maximum for the two periods ending August 26 and September 11. The maxima should indicate periods of most favorable moisture conditions and the minima should indicate those of relative drought The Easton graph for this ratio also shows two maxima (one for the period ending July 6 and the other for that ending August 31) but these do not correspond, in the periods of their occurrence, with the maxima shown for 


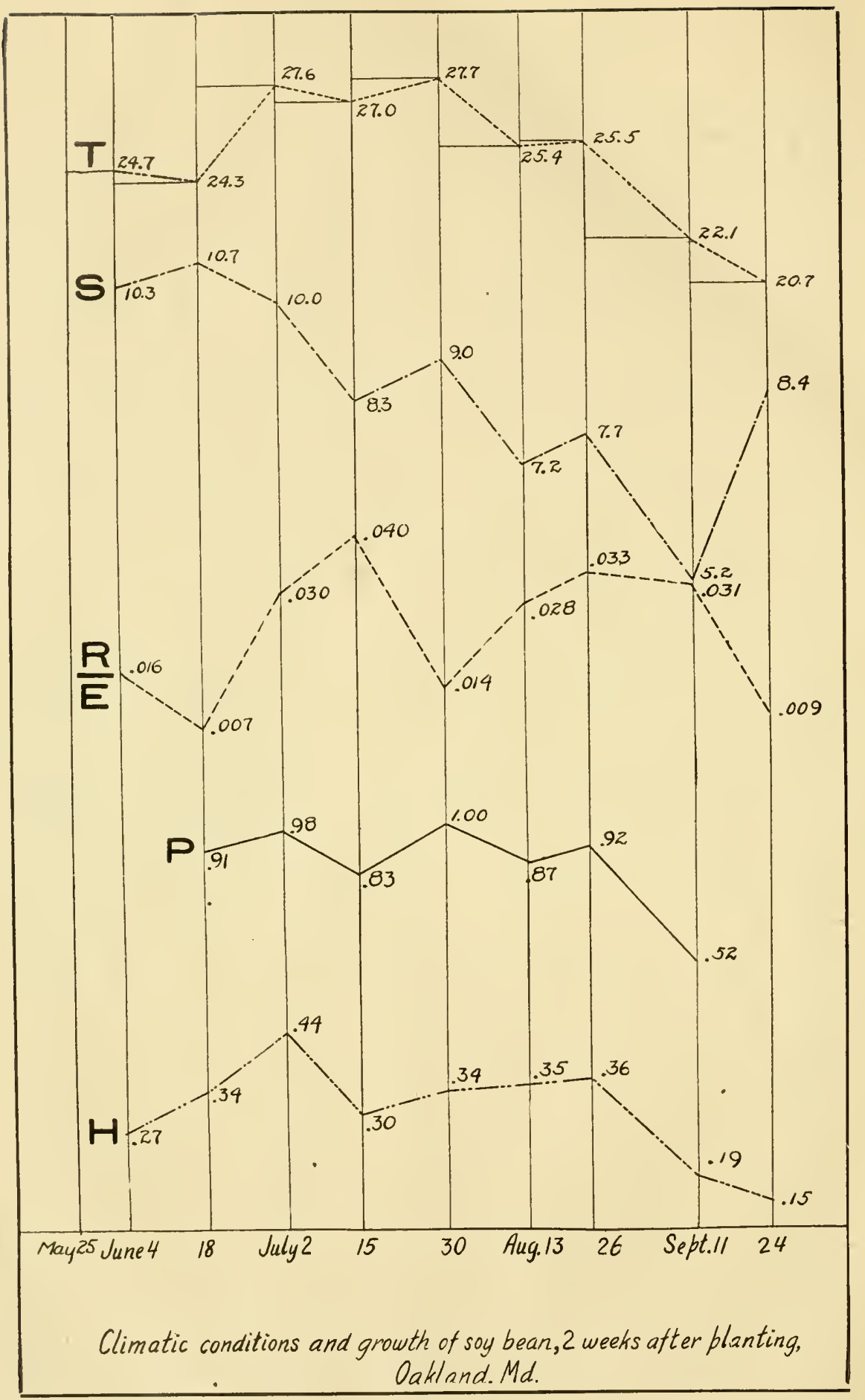

FIG. 9. $T$, temperature efficiency; $S$, sunshine duration; $\frac{R}{E}$, rainfall-evaporation ratio; $P$, leaf-product; $H$, stem height 


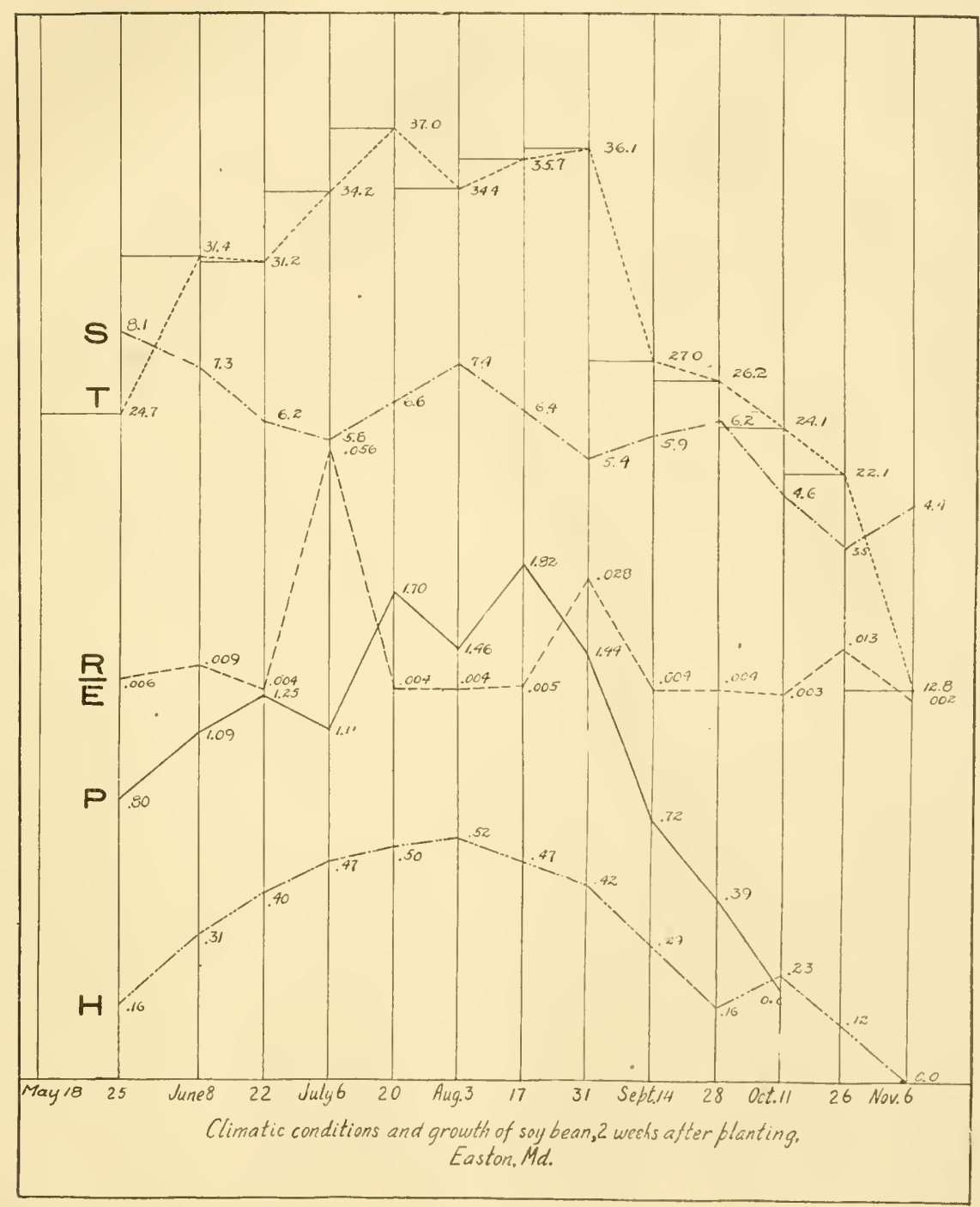

FJG. 10. $T$, temperature efficiency; $S$, sunshine duration; $\frac{R}{E}$, rainfall-evaporation ratio; $P$, leaf-produet; $H$; stem height 
Oakland, and the Easton graph is otherwise generally nearly horizontal It is noteworthy that the long-continued low values of this moisture ratio for Easton are markedly lower than the lowest values for the other station, also that the first maximum value for Easton is higher than either maximum for Oakland. It thus appears that the moisture conditions at Oakland were generally more favorable than those at Easton but that they were subject to greater extremes of fluctuation at the latter place. An interesting point is brought out when the graph for the moisture ratio is compared with that for sunshine duration, for the same station, in that a strong eorrespondence exists between these two graphs. Low values of the rainfall-evaporation ratio correspond generally to high magnitudes of the sunshine value, and conversely, for both stations. The moisture graph appears strikingly like what the sunshine graph would be if it were inverted. On days of prolonged sunshine the rainfall influence was low and the evaporation influence was high.

Comparison of graphs of growth rates with those of climatic conditions, for Oakland and for Easton. 'The graph of daily increase in leaf-product for' Oakland (fig. 9) shows the same direction of slope as does the one for average daily mean effective temperature, from point to point throughout the season. To the extent to which the sunshine graph agrees in direction of slope, with that for temperature, it also shows the same directions of slope as do the corresponding sections of the graph for leaf-product. There appears to be no relation between the graph of leaf-product and that of the rainfall-evaporation ratio for Oakland. The graph of the average daily increase in height is similar to that of leaf-product for all periods except the sixth (ending Aug. 13). For this period the graph of leaf-product shows a marked decrease from the previous period, while that for stem height increases slightly. Here the graph for leaf-product follows the direction of the temperature efficiency graph, as in the ease of the other periods.

The graphs of effective temperature, of leaf-product and of stem height, for Easton (fig. 10), also show a general similarity in their seasonal marehes. The sunshine and effective temperature graphs do not agree in trend for this station, as they do to a considerable extent for Oakland, and the graphs of growth rates show little tendency to conform to the variations in the direction of the sunshine graph. It may be noted here that the pronounced downward slope of the temperature graph does not begin until after the beginning of the corresponding decline in the graph for leaf-product. The temperature value continues high for about two weeks after the value of the leaf-product begins its downward trend. The decline in the graph of stem height begins even earlier than that in the graph of leaf-product.

In conclusion, it may be said that both of the graphs of growth rate for soy-bean seedlings within the first two weeks after planting exhibit distinct seasonal marches, which very closely resemble those of the corresponding 
graphs for the temperature efficiency values. No apparent relation is noticeable between the two graphs of growth rates, on the one hand, and those representing the other two elimatic factors, on the other. During the first two weeks after planting, the seedlings germinated and their stems elongated rapidly. The first pair of leaves were formed, but usually were not fully developed, at the time of the first measurement (approximately two weeks after planting). Thus, growth during this early period consisted largely in stem elongation, and seems in these experiments to have been most influenced by temperature. Since most of this development must have been accomplished at the expense of stored materials in the seed, and since an abundance of soil moisture was at all times supplied by the autoirrigators, the principal factors affecting the rate of development of the plants were probably the rate of hydrolysis of stored materials and the rate of conduction of the latter from the cotyledons to the growing points. Since such processes, and also those of growth itself, are profoundly influenced by temperature, it is not surprising that correlations with temperature conditions are the only ones brought out by these graphs. It is not to be inplied, however, that temperature was here the only influential factor, although it appears to be the most influential one, as far as these studies show.

SEASONAL MARCHES BASED ON PERIODS OF APPROXIMATELY FOUR WEEKS

Plant growth rates. As previously stated, each of the cultures was continued for a second period of two weeks, at the end of which time the plants were again measured. The behavior of the plants during this latter period of two weeks was somewhat different from that during the first period. The leaves expanded much more rapidly during the second fortnight than during the first, so that a considerable leaf surface was finally developed. In most instances the cotyledons remained attached to the plants throughout the entire month of growth, but they became yellow in many cases and probably usually became practically devoid of stored materials by the end of the month; in a few instances they fell from the plants before the time to harvest. Thus, with altered form and somewhat modified nutrition, the conditions influencing the rate of development of the plants might be expected to produce different results in the second two-week periodfrom those produced in the first.

The most important daily increments obtained from these final measurements (tables 2 and 4) are shown graphically in figures 11 and 12 , which are similar to figures 9 and 10 . Two additional sets of measurements, not available for the two-week periods, were taken at this final observation, those of actual leaf area and those of dry weight. The data of leaf area, obtained from photographic prints, is to be regarded as a true measure of the extent of the leaf surface at the end of the four-week period. The leaf- 
product was also obtained for the four-week periods, and, as has already been shown, these two measures of leaf area have a nearly constant ratio to each other, so that the relative rates of increase in leaf surface for the same plants during the two lengths of observation period may be directly compared by means of the leaf-product. Therefore the graphs showing rates of increase in leaf-product are given in figures 11 and 12, and the graphs of actual areal increase are not presented.

The data of dry weight of tops are especially important, since these are the only values obtained that furnish information on the approximate daily rates of accumulation of non-aqueous materials in the plants, and the graphs of average daily rate of increase in dry weight are included in figures 11 and 12 .

In the case of Oakland the graphs of daily increase in leaf length, leaf width and total number of leaves, for these later measurements, were found to show a seasonal trend similar to that exhibited by the leaf-product, and they showed little variation in this respect for Easton. Hence these three criteria are not shown by the graphs of figures 11 and 12, and the graphs for daily increase in dry weight of tops, leaf-product and stem height are the only ones referring to plant growth given in these figures.

Each of the graphs of average daily growth rates for the.four-week periods for Oakland (fig. 11), as well as each one for Easton (fig. 12), exhibits a seasonal march similar to that shown by the corresponding graph for the two-week periods. These three four-week graphs are similar in form for Oakland, being generally convex upward, but they all show concavity upward in the region of the fourth and fifth periods, this concavity being only slightly evident in the case of the graph for rate of increase in dry weight. The latter rises to its maximum for the third four-week period and then descends to the end of the season, with but a slight rise for the period ending August 13, while each of the other two shows two maxima.

For Easton, the graphs of increase in dry weight and in leaf-product agree with the one of increase in leaf-product for Oakland in showing two maxima, with a concavity between them, and the graph of increase in dry weight for Oakland shows the first of these maxima, but the dates of these maxima are not the same for the two stations. While these two periods giving low values of the leaf-product for Oakland were those ending July 30 and August 13, the corresponding low values for Easton (of both weight and product) are for the periods ending July 20 and August 3. There thus appears to have been a difference of ten days in the dates corresponding to these low values of the graphs, the upward concavity occurring earlier at Easton. In this connection it must be remembered, however, that records were obtained only at approximately two-week intervals, so that the exact dates corresponding to these two low values, or to the two maxima to which they are related, cannot be accurately fixed by the data at hand. 
The graph of average daily increase in stem height for Easton shows a single maximum near the center of the season, for the sixth period,

Climatic conditions. As has already been noted, the weather data for the four-week periods corresponding to the cluration of the cultures represented in the final measurements of the plants, are all derived from the data for the two week periods, to which they are naturally similar. The graphs of the three average daily values used for the fort night periods (temperature, sunshine and rainfall-evaporation ratio) are shown for the longer periods in figures 11 and 12, these three graphs being again comparable as to directron of slope and position of maxima and minima, but not as to the actual heights of their ordinates. The temperature graphs are of course smoother in this case than in that of the two-week data. The converse correspondence between the graph of the moisture ratio and that of sunshine duration is not as striking here as for the two-week periods, but is nevertheless apparent.

Graphs of growth rates compared with those of climatic conditions, for Oakland and for Easton. The graphs of plant growth (figs. 11 and 12) show no apparent relation to the marches of either rainfall or evaporation alone, but they do exhibit some interesting agreements with the march of the rainfall-evaporation ratio, which is graphically shown in these figures. While several periods for Easton show low sunshine values as concomitant with high values of leaf-product and dry weight, and while one such correspondence is evident in the case of Oakland, yet the failure of this relation to be general and the somewhat unsatisfactory nature of the sunshine data render the relation itself somewhat questionable. Of course it may sometimes oceur that too strong sunshine may retard plant growth throngh the moisture relation, and thus bring about such a correspondence as thist just mentioned. The rainfall-evaporation ratio (representing the moisture relation) will receive attention below.

For the data obtained two weeks after planting (figs. 9 and 10) there is no obvious agreement between the seasonal trend of the plant measurements and that of either sunshine duration or rainfall-evaporation ratio, but there is a general agreement between the seasonal mareh of effective temperature $(T)$ and that of stem height $(H)$ for both stations, as has been pointed out. Similarly, the graph of leaf-product $(P)$ for Easton shows a pronounced parallelism to that of effective temperature, but this agreement is not show $n$ for Oakland. It thus appears that the temperature conditions were the main controlling factor in the first two weeks of stem elongationforboth Oakland and Easton, but that this temperature control was not precise, some influence being exerted by other factors. It also appears that leaf expansion during the first 2 weeks of growth (measured by leaf-product) was rather definitely controlled by temperature conditions at the Easton station, but that other factors were not without influence upon this process at this station, these other factors constituting the main control at Oakland. 


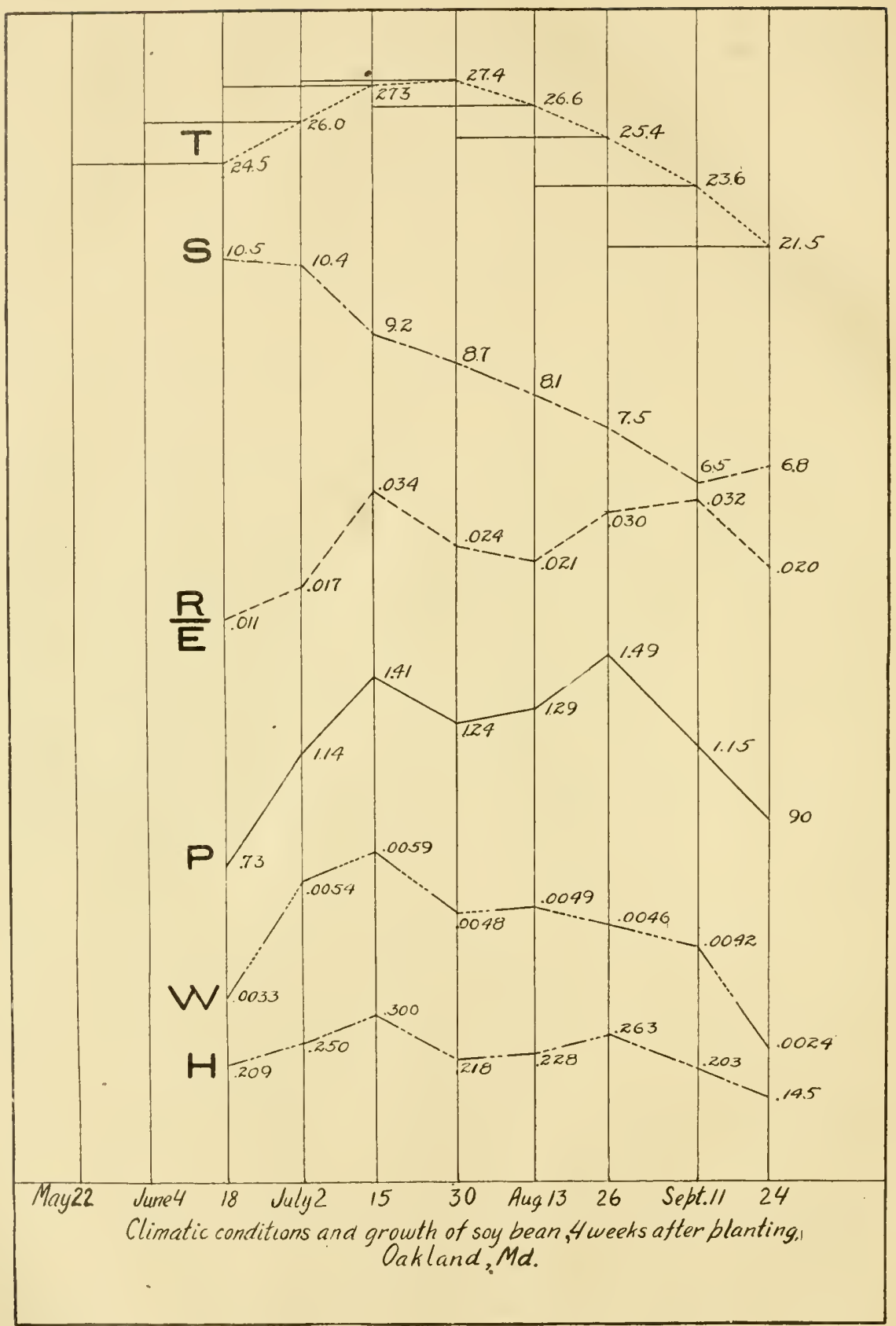

FIG. 11. $T$, temperature efficiency; $S$, sunshine duration; $\frac{R}{E}$, rainfall-evaporation ratio; $P$, leaf-product; $W$, dry weight; $H$, stem ${ }^{r}$ height 


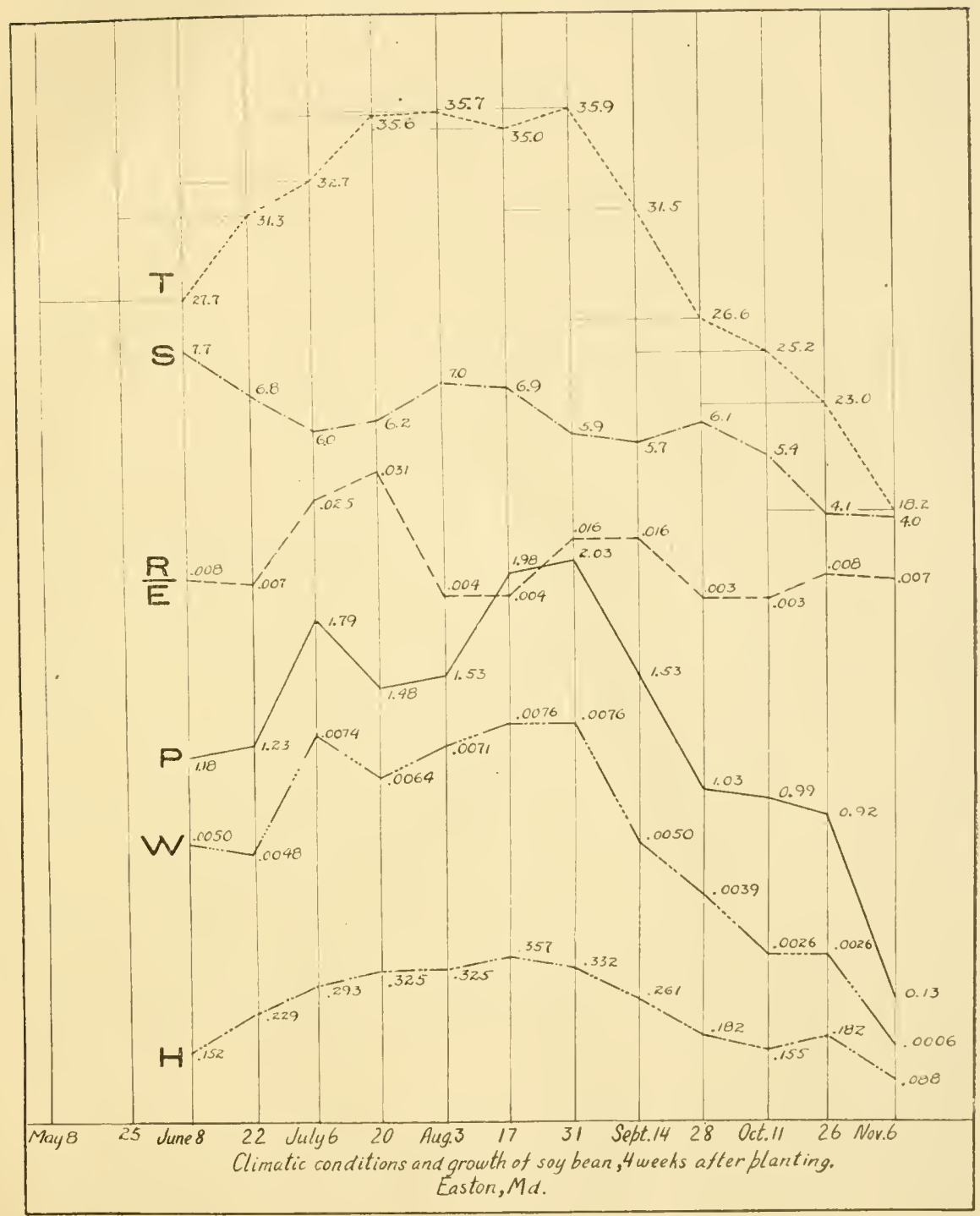

Fic. 12. $T$, temperature efficiency; $S$, sunshine duration; $\frac{R}{E}$, rainfall-evaporation ratio; $P$, leaf-product; $W^{r}$, dry weight; $H$, stem height 
In the case of the data obtained 4 weeks after planting (figs. 11 and 12), the graph of temperature conditions shows a general similarity to all three plant graphs, for both stations, but this agreement is only general. The plant graphs for leaf-product $(P)$ and stem height $(H)$ for Oakland agree with each other in showing two maxima (periods en ding July 15 and Aug. 26), and the first of these maxima is also shown by the graph of dry weight $(W)$. None of these maxima coincides in date with the single maximum of the graph of temperature efficiency. For Easton, the discrepancies of detail between the plant graphs and the the'graph of temperature efficiency for the 4 -week periods are fully as pronounced as those just mentioned. The graphs of leaf-product and weight both show two maxima (periods ending July 6 and Aug. 31), while this double maximum is barely indicated on the temperature graph. The graph of height fails to agree with the irregularities of either of the other two plant graphs and agrees only in its general seasonal march with the temperature graph. The four-week graph of sunshine shows no parallelism with any of the plant graphs in the case of either station.

A study of the four-week graphs for Oakland (fig. 11) brings out the fact that the two maxima of each of the graphs of leaf-product and stem height coincide in time of occurrence with high points on the graph of rainfallevaporation ratio and the depression between the two maxima on these growth graphs is also seen on the moisture graph. The second maximum of the moisture graph occurs a fortnight later than in the case of the graphs of leaf-product and stem height, but the upward slope of the moisture graph is slight for this fortnight. Also, the single maximum of the weight graph coincides in time with the first maximum of the graph of the moisture ratio. It thus appears that it is only for the beginning and end of the season that the direction of slope of the four-week plant graphs is generally the same as that of the corresponding temperature graph; during the middle portion of the season the plant graphs show a strong tendency to follow the direetion of the moisture graph, even where this graph differs radically from that of temperature. It may be said, for Oakland, that the four-week rates of increase in leaf-product and in stem height follow the four-week moisture ratio for that portion of the season when the four-week temperature efficiency value is above about 25 .

For Easton (fig. 12) the four-week data show a similar set of agreements and disagieements. In this case the height graph has nearly the sane slope throughout as has the temperature graph, but the other two plant graphs show pronounced disagreements with the graph of temperature, excepting at the end of the season. It is suggested that these clisagreenents may be controlled by moisture conditions, but the two maxima of the four-week moisture graph do not synchronize with those of leaf-product and weight; the moisture ratio maxima occur a fortnight later. Here, again, it may be said that the plant graphs generally agree in direction of slope with the tem- 
perature graph, from period to period, only when the temperature effieiency value is below about 25 .

From the two-week and four-week graphs of figures 9-12 it may be tentatively concluded (1) that the temperature relation is the main controlling factor for the growth rates based on the 2-week periods, no suggestion being apparent as to just what faetors besides temperature may have been influential; (2) that temperature conditions are the controlling or limiting factor for growth rates based on the four-week periods, as long as the temperature efficieney values are not above about 25; and (3) that the moisture conditions (represented by the rainfall-evaporation ratio) seem to have exerted a marked influenee upon the four-week growth rates during the time when the temperature efficiency values were above about 25 . From the fact that the influence of the moisture conditions is not apparent for the two-week data and is apparent or strongly suggested for the four-week data, it appears probable that the moisture ielation is relatively more important in the later stages of the development of the plants than in the earlier ones. If this supposition be true it may explain why the growth rates tend to follow the fluctuations of the moisture ratio as the plants become older. In such a case it should be the moisture conditions of the last 2 weeks of each of the four-week periods that are influential in determining the average growth rates for the four-week periods. To test this supposition, the fourweek data of leaf-product and the rainfall-evaporation ratios corresponding to the last two weeks of each four-week period have been brought together for comparison in the graphs of figures 13 and 14, the former for Oakland and the latter for Easton. In these figures the four-week leaf-product graph $(P)$ is the upper one, being reproduced from figure 11 or 12 . The fourweek temperature graph $(T)$ lies next below, also derived from figure 11 or 12. The third graph presents the two-week data of the rainfabl-evaporation ratio $\left(\frac{R}{E}\right)$ and is reproduced from figure 9 or 10 . The length of period represented by each of the successive values of the leaf-product and of the moisture ratio is shown by a horizontal line extending to the left of each point on these graphs.

Figures 13 and 14 inclieate a pronounced parallelism between the fourweek graph of leaf-product and the 2-week graph of the moisture ratio (for the last half of the four-week period), for both stations, thus furnishing evidence in favor of the supposition set forth above. It appears that the general rate of growth of these plants was influenced by temperature throughout the entire four-week period, but that the rate of leaf expansion was definitely influenced also by the moisture relations effective during the last 2 weeks of that period.

It seems reasonable to suppose that as the plant grows larger its sensitiveness to ehanges in its moisture surroundings increases; the greater expanse 


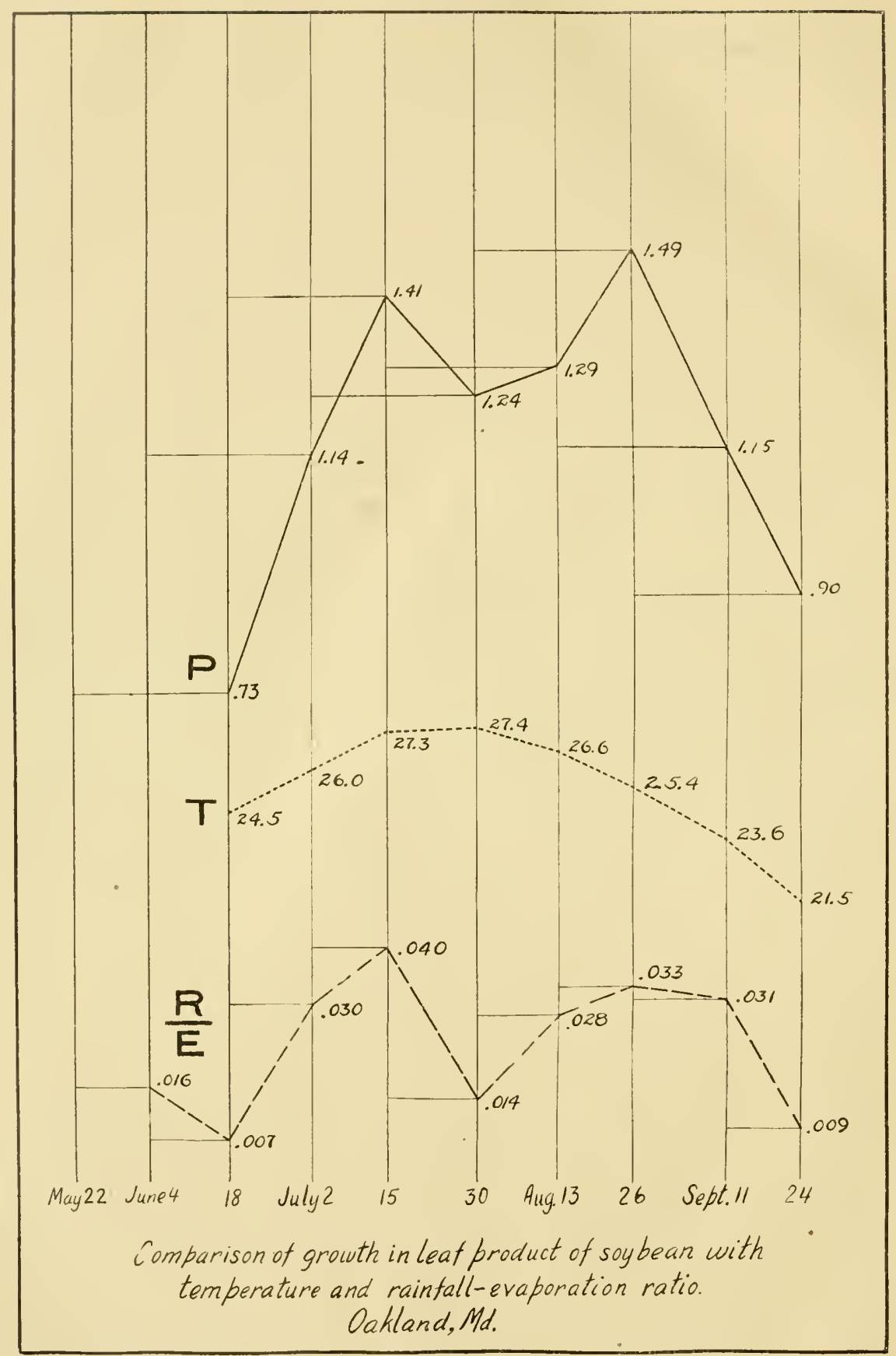




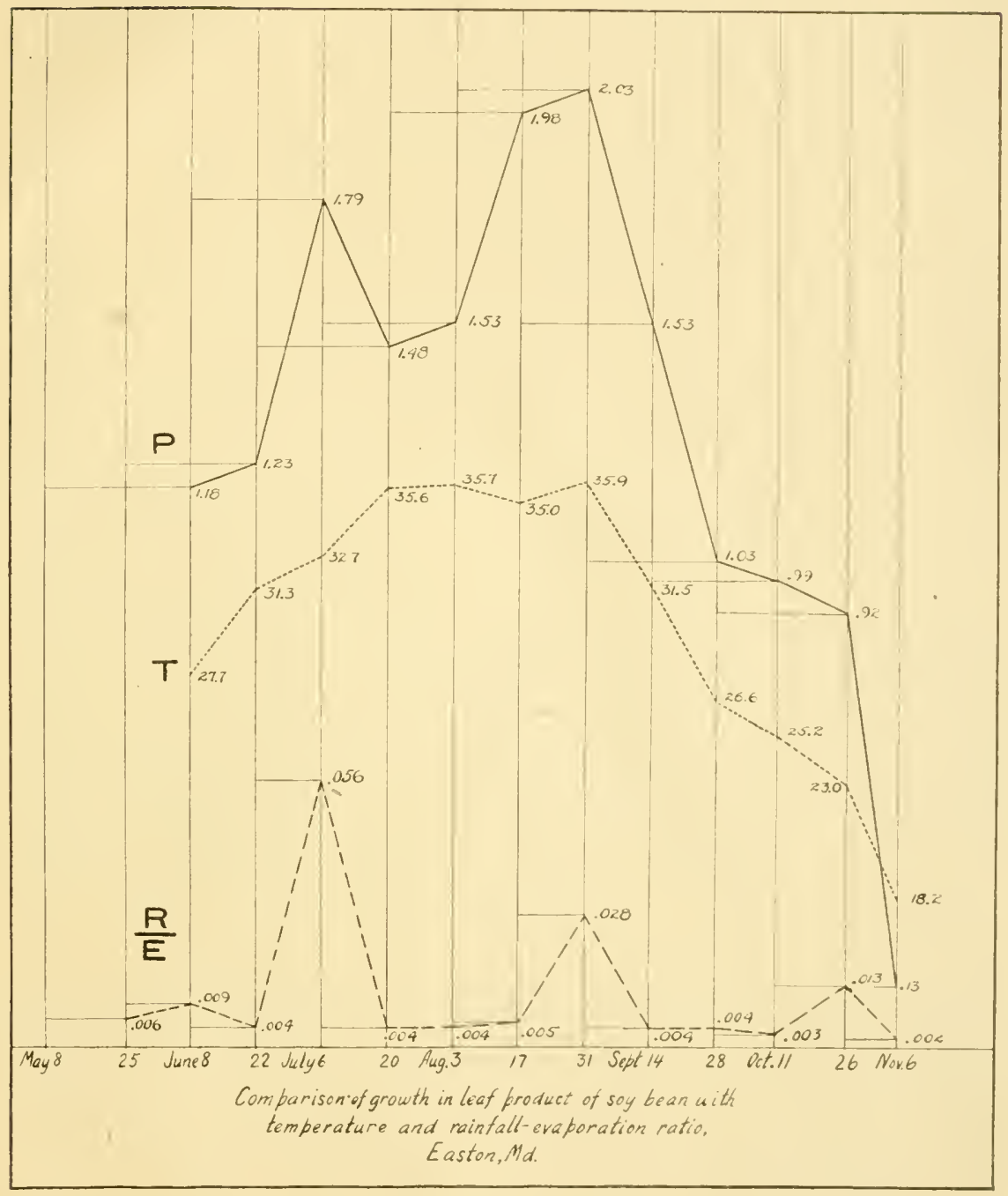

FIG. 14. $P$, leaf-product; $T$, temperature efficieney; $\frac{R}{E}$, rainfall-evaporation ratio 
of surface should be accompanied by an increased requirement for water to suipply that lost by transpiration. If this be granted it follows that the moisture conditions of the environment might be more than adequate for the early stages of development, while for later stages these same conditions might be limiting factors. ${ }^{32}$ This condition of affairs is suggested by the relation just brought out, between the moisture ratio and the rate of increase in leaf expanse. These plants never experienced pronounced drought, for they were always abundantly supplied with soil moisture and the evaporation intensities of this region are never very high. There were slight fluctuations in the moisture conditions, however, and if these were to affect the plants at all this should occur when the requirement for water is greatest; namely, in the later stages of development, when a relatively large surface is exposed to the air and sunshine. Furthermore, since rapid transpiration tends to deplete the water-content of leaves more than that of stems, it follows that leaf-growth should be retarded more than stem-growth, as the plant begins to experience a moisture deficit. The data of the present studies indicate that the culture plants were not markedly limited by the moisture conditions during the first two weeks of their growth from the seed, but moisture conditions did limit leaf expansion in the second twoweek period from the seed. 'The moisture conditions here varied but little, but they seem to have varied enough to influence the plants when the latter were most sensitive to them, and this influence became most markedly evident in that growth process (leaf expansion) which should be the most sensitive to these conditions. It may be added that this paramount influence of the moisture conditions would be expected to become evident especially in periods of high temperature, when transpiration should be most accelerated. With lower temperatures (efficiency values below about 25 , according to these data) the moisture conditions should lose their power to influence the plant, and temperature should become the main controlling condition. This is in accord with the facts above brought out.

These various considerations may be summarized as follows. (1) Temperature exerts an influence at all times. (2) With low temperatures this influence is the controlling one, but with high temperatures the moisture relation becomes important. (3) When this occurs, it is the moisture conditions of the last 2 weeks that control the average rate of leaf expansion for a four-week period.

A response in the growth rate to either one of two sets of climatic conditions

32 On the general theory of limiting conditions and its application, see the following:

Blackman, F. F., Optima and limiting factors. Ann. Bot. 19: 283-295. 1905.

Mitscherlich, E. A., Das Gesetz des Mlinimums und das Gesetz des abnehmenden Bodenartrags. Landw. Jahrb. 38: 537-552. 1909. Idem, Ueber das Gesetz des Minimums und die auf diesem ergebenden Schlussfolgerungen. Landw. Versuchsstat. 75: 231-263. 1911.

Smith, A. M., On the application of the theory of limiting factors to measurements and observations of growth in Ceylon. Ann. Roy. Bot. Gard., Peradini ya 3II: 303-375. 1906. 
within the same extreme limits of environmental conditions, such as is indicated in this case for the four-week periods, was somewhat similarly demonstrated by Smith (1906), for shorter observation periods, in a study of the relation of climatic conditions to the growth of the giant bamboo in Ceylon.

As has already been mentioned, the two-rveek data of sunshine duration correspond in an inverse manner to the data of the moisture conditions, and it follows that wherever the latter conditions appear to have controlled growth it also must appear that sunshine duration was an influential factor. The plants generally grew more with low sunshine values and less with high sunshine values. This somewhat unexpected observation seems to agree with the general physiological fact that plants actually grow most during the hours of darkness or of weak light, and it points clearly to the conclusion that the controlling influences of sunshine in these studies was exerted through the water relation. It has been suggested ${ }^{33}$ that physiological retardation of growth by light is really largely due to increased transpiration during the daylight hours. If the sunlight influence noted above were exerted upon the photosynthetic process it would be expected to have the opposite direction to that actually indicated; the plants should grow more during periods having high sunshine values. But they are here found to grow less during such periods.

In this general connection it is to be remembered, however, that the sumshine data here employed are probably not as reliable as the other climatic data. Of course other conditions than sunshine duration are influential in determining the value of the moisture ratio, but the relation here brought out emphasizes the apparent importance of sunshine as a factor in the water relation of plants. This matter will repay serious study whenever adequate methods for measuring sunshine may become arailable.

\section{CONCLUSIONS}

The study here reported was undertaken mainly to test, in a preliminary way, certain newly devised methods for attacking the general problem of the relation of plant growth to elimatic conditions. The results given in the preceding pages show that some of these new methods are of value, and the data obtained by their means throw light upon the question of the influence and relative importance of several different elimatic features, as these affected the growth of the culture plants.

The method here employed, of growing plants from seeds, as like as possible, in pots of like soil, for approximately equal short time periods at different stations, proved very satisfactory as a means of comparing climatic conditions for different localities and for different seasons of the year, as

${ }^{33}$ Palladin, W., Pflanzenphysiologie. Berlin, 1911. P. 257. 
these conditions influence plant growth. Such culture plants may be regarded as integrating instruments for the measurement of climate. They are started from the resting condition, as seeds (in which state they may be considered as instruments set at the zero points of their scales), and the amount of growth accomplished after any given period of exposure may be taken as the summed result of all the environmental influences that have acted upon the plants during the period. Unlike many of the man-made contrivances employed as measuring instruments, the standard plants cannot be reset at the zero point after reading, but must needs be replaced by new and similar individuals in the resting stage. Errors due to individual variations in the plants, introduced by thus using a succession of standard plants, were not found to be excessive.

The method here employed for soil moisture control, employing autoirrigators, proved very satisfactory for the purpose, although the details of this technique are susceptible of considerable improvement.

The methods by which the environmental conditions were measured in these studies were generally those in common use for similar purposes, and they require little comment here. The measurements of the evaporating power of the air, obtained with standardized cylindrical porous cup at mometers, taken with the ordinary precipitation records or measurements of rainfall gave a ratio of rainfall to evaporation that appears to be a very valuable measure of the environmental conditions as far as the water relation of the plants is concerned. Similarly, the records of daily maximum and minimum temperature readings, as obtained from maximum and minimum thermometers of the type now in common use by the U.S. Weather Bureau, were found to be entirely adequate for all the needs of temperature measurement encountered in this work. Concerning the sunshine records as here employed, obtained with the Marvin sunshine recorder, it appears that such data promise to be of some value in indicating the relative influence of sunshine duration in determining the magnitude of the rainfall-evaporation ratio, but no other relation between this climatic feature and the growth of the plants was discoverable.

Two methods of temperature summation were used in this study. One of these employs the direct summation of the daily mean temperatures above a certain assumed zero-point for growth, in this case above $40^{\circ} \mathrm{F}$. The second is an indirect method, using temperature efficiencies derived from the application of the chemical principle of van't Hoff and Arrhenius, in place of the actual temperature readings above an assumed physiological zero. The two methods agree in showing a clear relation between temperature and plant growth at both Oakland and Easton, but no evidence was brought out as distinctly in favor of either of the two methods.

The general conclusions of this study, regarding the relation of temperature, moisture and light conditions to the growth of these soy bean seedlings, 
have been summarized in the Abstract, at the beginning of the paper. While these conclusions constitute only a beginning, it appears that further study along lines similar to these may eventually develop a considerable knowledge of the relations here dealt with, and that this knowledge may furnish an important point of view for future climatological work. It promises, also, to be of practical value in connection with agriculture and forestry. In the planning of further studies, it must be borne in mind, however, that the problem is a very complex one, which cannot be expected to yield to any simple treatment. Nevertheless, it seems that climatology and plant physiology are now far enough advanced to warrant serious and sustained attack upon this very important question of the relation of climatic conditions to plant growth and development.

\section{LITERATURE CITED}

AвBE, C., First report on the relation between elimate and crops. U. S. Weather Bur. Bull. 36.1903. Arctowski, HeNryk, Studies on elimate and crops: corn erops of the United States. Bull. Ainer. Geog. Soe. 44: $745-760.1912$

Blackina, F. F., Optima and limiting faetors. Ann. Bot. 19: 2S3-295. 1905

Bovste el, J. A., The soils of Prince George's County. Maryland Geologieal Survey. Baltimore, 1911.

B. SHANtz, Daily transpiration during the normal growth period and its eorrelation with the weather. Jour. Agric. Res. 7: 155-212. 1916.

Brows, W. H., The relation of evaporation to the water content of the soil at the time of wilting. Plant World, 15: 121-134. 1912.

Cosibes, R., La determination des intensités lumineuses optima par les vegetaux au divers stades de developpement. Ann. Sci. Nat. Bot. IX, 11: 74-254. 1910

FAssig, O. L. The period of safe plant growth in Maryland and Delaware. Monthly Weather Rev. 42: 15215S. 1914.

HAW

HILGARD, E. W., Soils, their formation, properties and eomposition. New York, 1911.

Jor time intervals. Plant World 19: 136-140. 1916.

Firssebach, T. A., Transpiration as a factor in erop production. Nebraska Agric. Exp. Sta. Researeh Bull. 6. 1916.

Kimball, H. H., The total radiation reeeived on a horizontal surface from the sun and sky at Mount Weather. Monthly Weather Rev. 42: 474-187. 1914.

liomppen, W., Wärmezonen der Erde, nach der Dauer der heissen, gemässigten und kalten Zeit und nach de Wirkung der Wärme auf die organische Welt betraehtet. Meteorol. Zeitsehr. 1: 215-226. 1884.

Lehenbader, P. A., Growth of maize seedlings in relation to temperature. Physiol. Res. 1: 247-248. 1914.

—_ Light intensity and transpiration. Bot. Gaz. 52: 417-438. 1911.

- A rotating table for standardizing porous eup atmometers. Plant World 15: 157-162. 1912.

Climatic areas of the United States as related to plant growtl. Proc. Amer. Phil. Soc. 52: 25j-275. 1913.

Atmometry and the porous eup atmometer. Plant World 18: 21-30, 51-74, 95-111, 143-149. 1915.

(Wash. Pub. 204: 3-48. 1915.

Lriver Temperature coeffeients in plant geography and climatology. Bot. Gaz. 56: 346-375. 1913.

Lubisienzo, W., Produetion de la substanee seche et de la chlorophyll chez les vegetaux superieures aux differents intensités lumineuses. Ann. Sei. Nat. Bot. IX, 7: 321-415. 1908.

IAcDocgal, D. T., The auxothermal integration of elimatic eomplexes. Amer. Jour. Bot. 1: 1S6-193. 1914,

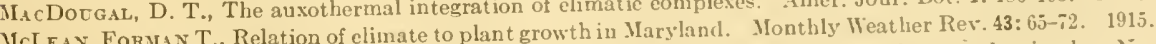

IERriam, C. HaRt, Laws of temperature control of the geographic distribution of plants and animals. National Geog. Mag. 6: 229-238. 1894

Jiтscherucr, E. A., Das Gesetz des Minimums und das Gesetz des abnehmenden Bodenertrags. Landw. Jahrb. 38: 537-552. 1909.

Teber das Gesetz des Minimums und die auf diesem ergebenden sehlussfolgerungen. Landw. Versuchsstat. 75: 231-263. 1911 . 
Palladin, W., Pflanzenphysiologie. Berlin, 1911.

Pulling, H. E., and B. E. Livingston, The water-supplying power of the soil as indicated by osmometers. Carnegie Inst. Wash. Pub. 204: 49-84. 1916.

Richter, A., Etude sur la photosynthèse et sur l'absorbtion par la feuille verte. Rev. gen. Bot. 14: 151-169, 211218. 1902 .

Rose, E., L'encrgie assimilatrice chez les plantes. Ann. Sci. Nat. Bot. IX, 17: 1-110. 1913.

Shive, J. W., An improved non-absorbing porous cup atmometer. Plant World 16: 7-10. 1915.

Shreve, F., Rainfall as a determinat of soil moisture. Plant World 17: 9-26. 1914.

Sмrт, A. M., On the application of the theory of limiting factors to measurements and observations of growth in Ceylon. Ann. Roy. Bot. Gard. Peradiniya 3II: 303-375. 1906.

Sмith, J. WARREN, The effect of weather upon the yield of corn. Monthly Weather Rev. 42: 78-93. 1914.

Transead, E. N., Forest centers of eastern North America. Amer. Nat. 39: 875-889. 1905.

- Apparatus for the study of comparative transpiration. Bot. Gaz. 52: 54-60. 1911.

Zon, R., Meteorological observations in connection with botanical geography, agriculture and forestry. Monthly Weatluer Rev. 42: 217-223. 1914. 


\section{VITA}

The writer was born Juue $2 \bar{\tau}, 1885$, at Colts Neck, New Jersey. He is the son of John Hull MeLean, and Eliza (Taylor) MeLean. He received his early school training in private schools, preparing for college at a public high school. He entered the Sheffield Scientific School of Yale University in 1904, graduating from that institution which honors in Forestry in 1907, and receiving the degree Ph.B. He attended the Yale Forest School the following year, and received the degree of Master of Forestry in 1908. In July, 1908, he entered the U.S. Forest Service as Forest Assistant, and was assigned to research work; first, in the Bald (ypress swamps of the Gulf Coast (1908-1909), and later in the Rocky Mountains (1909-1913). In 1912 he was placed in charge of the silvieultural investigations at the Utah Forest Experiment Station, as Forest Examiner. He was married on October 27, 1913, to Mary Osborn Morford, of Red Bank, New Jersey. In the autumm of 1913 , the writer undertook the stuly reported in the preceding pages, under the auspices of the Maryland state Weather Service, and with the gencral direction of Prof. B. E. Livingston. 

LIBRARY OF CONGRESS

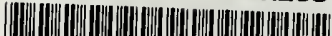

00025952789 